INTER NATIONAL MONETARY FUND

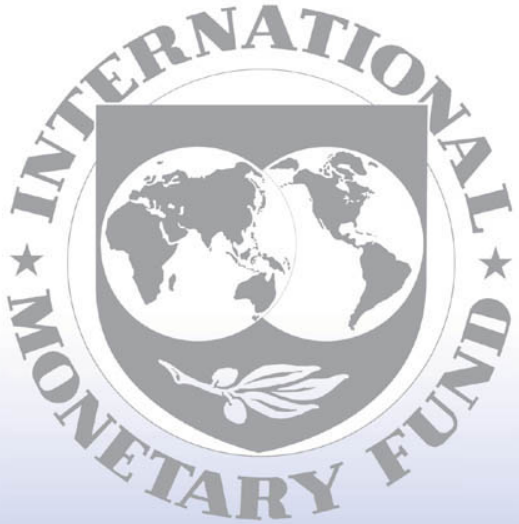

Staff

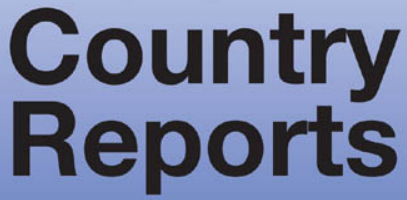


Democratic Republic of São Tomé and Príncipe: 2005 Article IV Consultation and First Review Under the Three-Year Arrangement Under the Poverty Reduction and Growth Facility-Staff Report; Staff Statement; Public Information Notice and Press Release on the Executive Board Discussion; and Statement by the Executive Director for the Democratic Republic of São Tomé and Príncipe

Under Article IV of the IMF's Articles of Agreement, the IMF holds bilateral discussions with members, usually every year. In the context of a combined discussion of the 2005 Article IV consultation with the Democratic Republic of São Tomé and Príncipe and first review under the three-year arrangement under the Poverty Reduction and Growth Facility, the following documents have been released and are included in this package:

- $\quad$ the staff report for the 2005 Article IV consultation and first review under the three-year arrangement under the Poverty Reduction and Growth Facility, prepared by a staff team of the IMF, following discussions that ended on November 19, 2005, with the officials of the Democratic Republic of São Tomé and Príncipe on economic developments and policies. Based on information available at the time of these discussions, the staff report was completed on February 15, 2006. The views expressed in the staff report are those of the staff team and do not necessarily reflect the views of the Executive Board of the IMF.

- a staff statement of March 6, 2006, updating information on recent developments.

- a Public Information Notice (PIN) and Press Release summarizing the views of the Executive Board as expressed during its March 6, 2006 discussion of the staff report that concluded the Article IV consultation and the IMF arrangement, respectively.

- $\quad$ a statement by the Executive Director for the Democratic Republic of São Tomé and Príncipe.

The document(s) listed below have been or will be separately released.

\section{Selected Issues Paper}

The policy of publication of staff reports and other documents allows for the deletion of market-sensitive information.

To assist the IMF in evaluating the publication policy, reader comments are invited and may be sent by e-mail to publicationpolicy@imf.org.

Copies of this report are available to the public from

International Monetary Fund • Publication Services

$70019^{\text {th }}$ Street, N.W. $\bullet$ Washington, D.C. 20431

Telephone: (202) 623-7430 • Telefax: (202) 623-7201

E-mail: publications@imf.org Internet: http://www.imf.org

Price: $\$ 18.00$ a copy

\section{International Monetary Fund} Washington, D.C. 
This page intentionally left blank

CInternational Monetary Fund. Not for Redistribution 
INTERNATIONAL MONETARY FUND

\title{
DEMOCRATIC REPUBLIC OF SÃO TOMÉ AND PRÍNCIPE
}

\section{Staff Report for the 2005 Article IV Consultation and First Review Under the Three-Year Arrangement Under the Poverty Reduction and Growth Facility}

\author{
Prepared by the African Department \\ (In consultation with other departments) \\ Approved by Jean A. P. Clément and Scott Brown
}

February 15, 2006

- On August 1, 2005, the Executive Board approved a three-year arrangement under the PRGF for São Tomé and Príncipe in the amount of SDR 2.96 million (40 percent of quota). So far, São Tomé and Príncipe has drawn SDR 0.423 million under the arrangement.

- Discussions on the 2005 Article IV consultation and First Review under the PRGF arrangement were held in São Tomé during November 5-19, 2005. The staff team comprised Messrs. Pastor (head), Iimi, Ronci, and Segura (all AFR). Mr. Randriamaholy, the Fund resident representative, assisted the mission. The mission met with the Prime Minister and Minister of Planning and Finance, the Minister of Natural Resources and Environment, the Acting Governor of the Central Bank of São Tomé and Príncipe (BCSTP), other senior government officials, as well as the President of the National Assembly, the Chairman of the Oil Commission at the National Assembly, representatives of the donor community, labor unions, and private sector businesses.

- The current government, in place since early June 2005, has expressed a strong commitment to implement the Fund-supported program with a view to reaching HIPC completion point by mid2006. Parliamentary elections, and the formation of a new government, are expected around April 2006, ahead of Presidential elections, which are scheduled for late 2006.

- In the attached letter dated February 9, 2005 (Attachment I), the authorities request the completion of the First Review and the disbursement of the second tranche under the PRGF in an amount equivalent to SDR 0.423 million, equivalent to 5.7 percent of quota. The memorandum of economic and financial policies (MEFP), attached to the letter, reviews economic policy implementation in 2005 and proposes performance criteria for end-June 2006.

- The Sãotomean authorities appreciated the advice provided by the Fund Executive Board in the context of the Article IV consultations. They particularly welcomed the Board's recommendation during the latest consultation to develop strong institutions with a view to secure a proper management of prospective oil revenue. The authorities stressed the need of continued technical assistance in the areas of Fund expertise to buttress administrative capacity and policy decision making. 
Executive Summary

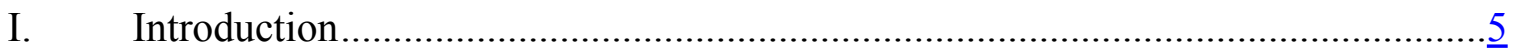

II. Recent Economic Developments and Performance under the Program .................

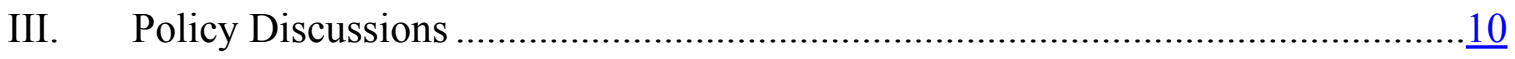

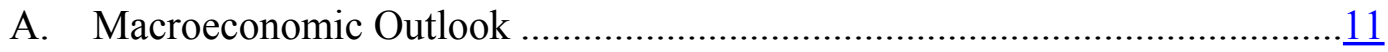

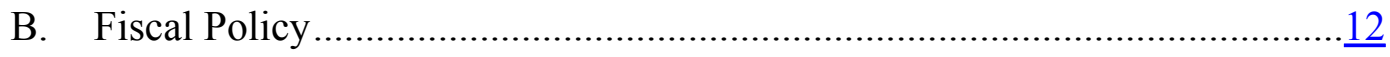

C. Monetary and Exchange Rate Policies ...................................................

D. Banking Sector............................................................................ 15

E. External Sector Policies ................................................................ 15

F. Structural Reform and Governance Issues....................................... 17

G. Poverty Reduction Strategy …...............................................................18

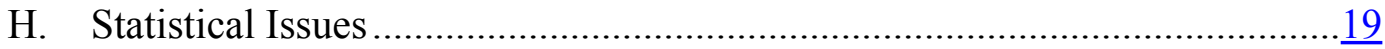

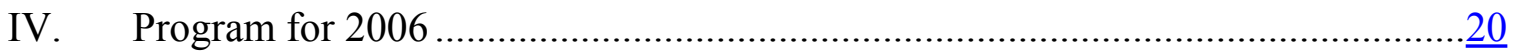

V. Program Monitoring, Risks, and Safeguards Assessment ...................................

VI. Staff Appraisal …................................................................................... 21

\section{Boxes}

1. Oil Sector Prospects...............................................................................

2. Revenue Measures in the Authorities' Medium-Term Fiscal Plan.......................13

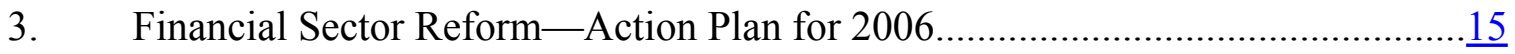

4. External Sector Policies — Status Report ................................................

Figures

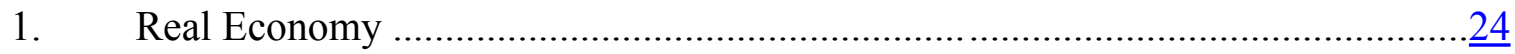

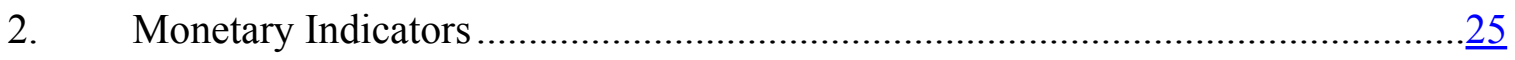

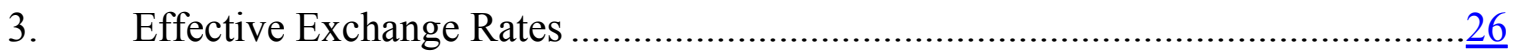

Tables

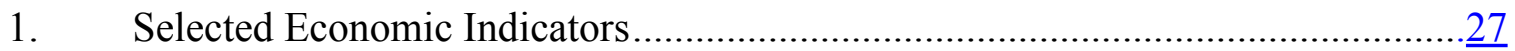

2. Financial Operations of the Central Government (In billions of dobras) ..............28

3. Financial Operations of the Central Government (In percent of GDP) .................29

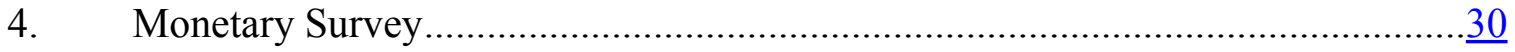

5. Summary Accounts of the Central Bank ......................................................... 


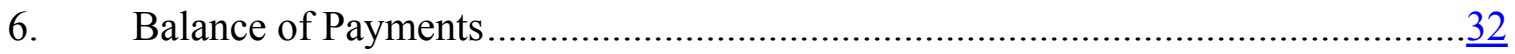

7. External Financing Requirements and Sources ................................................

8. Performance Criteria and Indicative Targets for 2005-06 ................................

9. Structural Performance Criteria and Benchmarks for First PRGF Review ............ $\underline{36}$

10. Structural Reform Program at a Glance.....................................................

11. Millennium Development Goals.............................................................. $\underline{38}$

12. Implementation Status of Floating Completion Triggers …............................. $\underline{40}$

13. Schedule of Disbursements Under the PRGF Arrangement...............................

14. Indicators of Fund Credit .......................................................................

Appendices

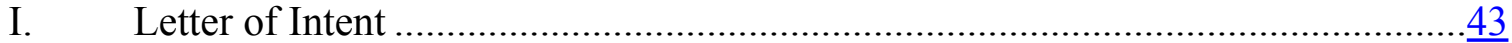

Attachment I: Memorandum of Economic and Financial

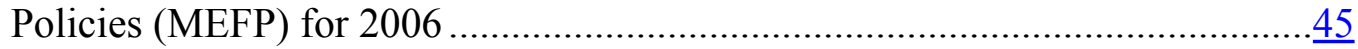

Attachment II: Technical Memorandum of Understanding............................... $\underline{58}$

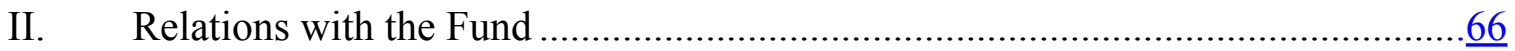

III. Relations with the World Bank Group.........................................................

IV. Relations with the African Development Bank Group ....................................... $\frac{72}{74}$

V. Debt Sustainability Analysis......................................................................

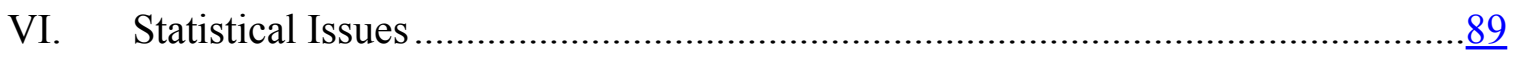

Statement by the IMF Staff Representative ............................................................ $\frac{94}{96}$

Public Information Notice................................................................................... $\frac{96}{01}$

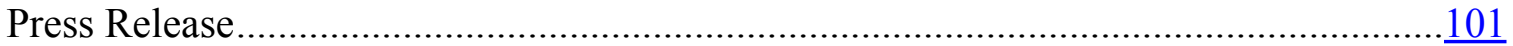

Statement by Mr. Ondo Mãne.......................................................................... 


\section{EXECUTIVE SUMMARY}

- $\quad$ Performance under the program supported by the PRGF has been broadly satisfactory to date. All quantitative performance criteria set for end-September 2005 were observed. In addition, all structural performance criteria set for end-July 2005 were met, and three out of four structural benchmarks were observed as scheduled. The submission to the National Assembly of legislation criminalizing money laundering and the financing of terrorism is still pending because of the need to update related pieces of legislation. However, the government intends to finish updating the legislation during 2006.

- São Tomé and Príncipe needs to continue to address macroeconomic imbalances and set the conditions for sustained economic growth and poverty alleviation. Fiscal consolidation, while preserving an adequate level of pro-poor spending, would be critical to sustain macroeconomic stability. Over the medium term, a main task would be to develop strong institutions to secure a transparent management of oil revenue. As the oil sector is likely to be an enclave, the authorities need to secure a proper execution of the government's Poverty Reduction Strategy Paper that would strengthen growth in the non-oil economy, reduce poverty, and support the attainment of the MDGs.

- In 2005, economic growth remained strong, although inflation picked up in 2005 against the background of a sharp increase in international oil prices that were passed-through to domestic consumers. Fiscal consolidation advanced in line with the program, and the fiscal targets for end-2005 are likely to have been met. Broad money grew rapidly on account of resilient economic growth and an increase in the central bank's NIR position. The managed floating exchange rate regime has served the country well and with the latest changes it better reflects market conditions. The import coverage of reserves remains above 3.5 months of imports of goods and services.

- Prospects for 2006 remain favorable, including strong growth, a reduction in inflation, and maintenance of a sustainable external position. The 2006 program seeks to consolidate the fiscal measures launched in 2005, including efforts to address tax arrears and overhaul customs administration procedures. A better control of the growth of current expenditure, particularly the wage bill, will help to change the composition of government spending in favor of pro-poor expenditure. Monetary policy will be consistent with a deceleration of inflation. To foster private sector growth in the non-oil economy, structural reform efforts will continue, including actions to address the financial position of the water and electricity company (EMAE).

- On program risks, a main concern is that the process of fiscal consolidation may be disrupted against the background of the elections scheduled for 2006. Policy credibility could also be damaged if the authorities fail to demonstrate their readiness to follow suit with ongoing investigations about alleged irregularities in the licensing round for oil Blocks 2-6. Moreover, possible shortfalls in foreign assistance triggered by the future oil boom could exacerbate the government's budget constraint in the short run. 


\section{INTRODUCTION}

1. Following a decade of large macroeconomic imbalances and state intervention in the economy, the Sãotomean authorities have pursued economic reforms since 1998 that have helped increase real GDP growth, lower inflation, and push forward key structural reforms. Financial aid from international donors increased substantially in support of these reforms. At the same time, a successful implementation of a staff monitored program in 1999 led to the approval of a three-year arrangement under the PRGF in April 2000. São Tomé and Príncipe reached the HIPC Decision point in late December 2000.

2. However, fiscal performance has been uneven over the years, reflecting expenditure pressures arising from the domestic political cycle and anticipated large oil signature bonuses. In 2001, as presidential elections approached, the government failed to meet a number of fiscal and monetary targets under the Fund supported program. Fiscal management improved in 2002, but it deteriorated again in 2003 as the government raised social spending significantly in the aftermath of a "coup d'état" in July 2003. In 2004, the fiscal position deteriorated even further as the government increased expenditures to unsustainable levels in anticipation of a large oil signature bonus which in the event was not received in 2004, but only in July 2005 in an amount significantly smaller than originally envisaged. Rising fiscal deficits in 2003-04 were financed mainly by short- and medium-term external borrowing, central bank financing, and an accumulation of external arrears.

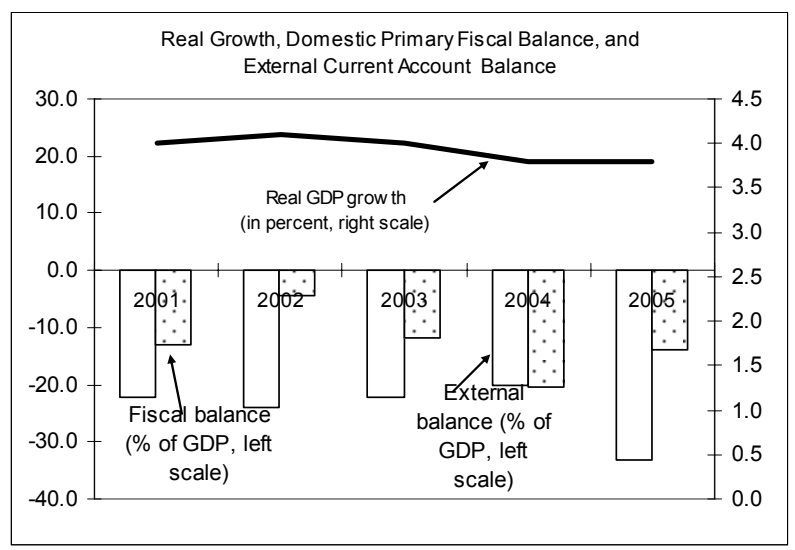

3. Political stability has remained a challenge in São Tomé and Príncipe since the 1990s, resulting at times in disagreements among senior civil servants belonging to different political parties. This has led to administrative deadlocks from time to time and to stop-and-go in economic policy implementation and reform.

4. The current PRGF arrangement, approved by the Executive Board on August 1, 2005, seeks to support the government's efforts to address macroeconomic imbalances and set the conditions for sustained economic growth and poverty alleviation. ${ }^{1}$ The policy agenda is threefold. First, São Tomé and Príncipe needs to continue fiscal consolidation efforts, while preserving an adequate level of pro-poor spending, to sustain macroeconomic stability. Second, it needs to reach the HIPC completion point through a steady implementation of its PRGF-supported program to reduce the external debt

\footnotetext{
${ }^{1}$ São Tomé and Príncipe's relations with the Fund are summarized in Appendix II. Relations with the World Bank and the African Development Bank are described in Appendices III and IV. The staff's debt sustainability analysis (DSA) and a summary of statistical issues are discussed in Appendices V and VI, respectively.
} 
service burden and liberate resources to increase pro-poor spending. Third, São Tomé and Príncipe needs to safeguard an effective management of the prospective vast oil revenue against the background of weak institutional and absorptive capacities (Box 1). As the oil sector is likely to be an enclave, reducing poverty and attaining the Millennium Development Goals (MDGs) would need high non-oil GDP growth supported by a thorough implementation of the government's structural reform program included in the PRSP.

\section{Box 1. São Tomé and Príncipe: Oil Sector Prospects}

- Geological analysis in the early 1990s identified large oil and natural gas deposits off-shore from the islands on São Tomé and Príncipe. The 2001 territorial agreement with Nigeria resulted in the creation of a Joint Development Zone (JDZ) to be administered by the Joint Development Authority (JDA) headquartered in Abuja. It was agreed that São Tomé and Príncipe would receive 40 percent of all oil revenue from the JDZ. An Exclusive Development Zone (EDZ), which has been recognized internationally, will be exploited solely by São Tomé and Príncipe.

- Oil revenue in the pre-production period will be in the form of oil signature bonuses paid by field operators. The tax regime, embodied in the Production Sharing Agreements (PSAs) that are being negotiated with oil companies, would determine the government's oil receipts during the production period starting around 2012/13. The oil signature bonus on Block 1 in the JDZ, totaling US\$49.2 million (equivalent to about 75 percent of GDP), was received in July 2005. Negotiations of PSAs for Blocks 2-6 are currently underway, with signature bonuses, totaling US\$54.7 million, expected during the first half of 2006.

- Preliminary staff estimates indicate that oil production could have a sizeable effect on São Tomé and Príncipe's economic prospects. The country's oil output share could be about 30 thousand barrels per day around 2013, under the rather conservative assumption that only one out of six blocks located in the JDZ is found to be commercially exploitable. Under this scenario, an assuming a constant real price of US\$30 per-barrel of oil, annual budget revenue from oil could stabilize at around US\$92 million over the long term (equivalent to 130 percent of 2006 GDP), with the Permanent Fund reaching a steady state level of US $\$ 3.1$ billion in constant 2006 dollar terms. If São Tomé and Príncipe's share of oil output were to reach 80,000 barrels per day, government budget receipts and the size of the Permanent Fund would roughly triple.

1/ Detailed analysis is presented in Chapters I and II of the accompanying Selected Issues Paper. 


\section{Recent Economic Developments and Performance under the Program}

5. Economic performance in $\mathbf{2 0 0 5}$ has been generally satisfactory, although inflation has picked up (Tables $1-7$ and Figure 1). Real GDP increased to 3.8 percent, while the 12-month CPI inflation rate rose to 17 percent in December 2005. Official estimates suggest that at least 2 percentage points in the current inflation rate are explained by higher international oil prices, which were passed through to domestic consumers. ${ }^{2}$ A higher- than-programmed depreciation of the dobra also contributed to the acceleration of inflation.

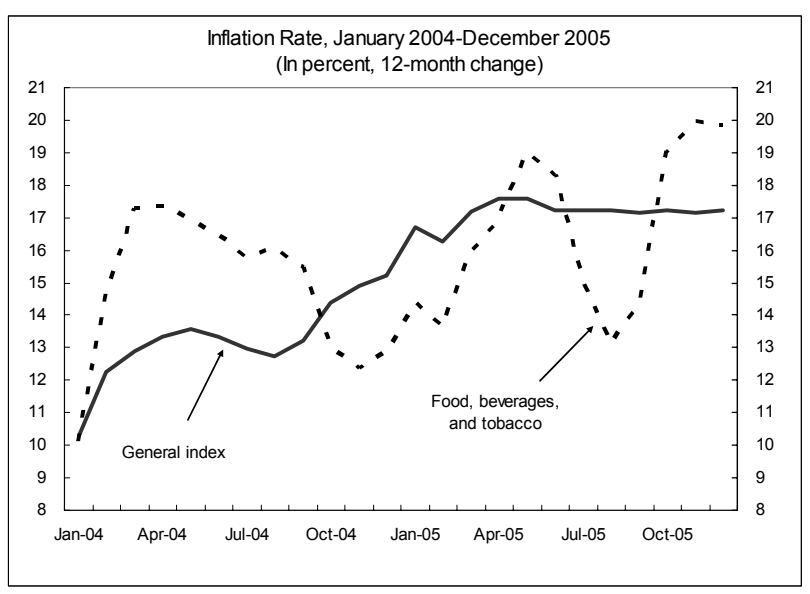

São Tomé and Príncipe: Key Macroeconomic Indicators, 1999-2005

\begin{tabular}{|c|c|c|c|c|c|c|c|c|}
\hline & \multirow[t]{2}{*}{1999} & \multirow[t]{2}{*}{2000} & \multirow[t]{2}{*}{2001} & \multirow[t]{2}{*}{2002} & \multirow[t]{2}{*}{2003} & \multirow[t]{2}{*}{2004} & \multicolumn{2}{|c|}{2005} \\
\hline & & & & & & & $\mathrm{CR} / / 05 / 323$ & Est. \\
\hline Real GDP (annual percentage change) & 3.3 & 3.0 & 4.0 & 4.1 & 4.0 & 3.8 & 3.2 & 3.8 \\
\hline Consumer prices (annual percentage change; end of period) & 12.6 & 9.6 & 9.4 & 8.9 & 10.2 & 15.2 & 15.0 & 17.0 \\
\hline Consumer prices (annual percentage change; average) & 16.3 & 10.7 & 9.5 & 9.2 & 9.6 & 12.8 & 15.1 & 16.2 \\
\hline Domestic primary fiscal balance (percent of GDP) & 1.3 & 2.1 & -12.9 & -4.3 & -11.7 & -20.6 & -17.5 & -14.0 \\
\hline Exchange rate (in dobras per U.S. dollar; end of period) & 7,250 & 8,568 & 9,020 & 9,192 & 9,456 & 10,104 & 11,396 & 11,930 \\
\hline \multicolumn{9}{|l|}{ External current account balance (percent of GDP) } \\
\hline Including official transfers & -62.0 & -52.3 & -25.2 & -24.1 & -22.3 & -20.1 & -28.2 & -33.1 \\
\hline Excluding official transfers & -58.0 & -52.3 & -63.1 & -54.5 & -56.7 & -58.9 & -60.3 & -59.2 \\
\hline NPV of external debt (in percent of exports of goods and services) & $1,402.2$ & 462.0 & 481.6 & 467.3 & 463.5 & 500.7 & 465.3 & 499.5 \\
\hline Gross international reserves 1 / & 3.1 & 3.5 & 3.9 & 3.9 & 4.8 & 3.5 & 3.4 & 3.7 \\
\hline
\end{tabular}

Sources: Sãotomean authorities and Fund Staff estimates.

1 / In months of following year's non-oil imports of goods and nonfactor services.

6. Performance under the program has been broadly satisfactory to date. All eight quantitative performance criteria set for end-September 2005 were observed (Table 8). Also, all structural performance criteria set for end-July 2005 were met. This included the submission to the National Assembly of (i) a new investment code that would provide equal treatment for domestic and foreign investors, (ii) a new tax code on personal income taxation that would broaden the tax base and update tax brackets to provide progressivity to the tax

\footnotetext{
${ }^{2}$ Gasoline prices were increased from US\$0.84 per liter to US\$1.04 in October 2004. Prices were increased again to US $\$ 1.23$ per liter in July 2005 . A new round of price increases is currently under consideration by the authorities.
} 
system, (iii) a new tax code on corporate taxation that would substantially limit the granting of tax holidays to new licensed investors, and (iv) a new tax procedural code establishing tax tribunals at different levels of government to handle tax issues and disputes, including tax penalties and fines. In addition, for end-September, three out of four structural benchmarks were observed (Table 9). The submission to the National Assembly of legislation criminalizing money laundering and the financing of terrorism (AML/CFT legislation) is still pending because of the need to update related pieces of legislation. However, the government intends to submit the legislation to the National Assembly by end-December 2006.

\section{The external current account deficit (excluding official transfers) in $\mathbf{2 0 0 5}$ is} expected to remain broadly unchanged with respect to 2004, at about 59 percent of

GDP. A worsening in trade balance due to a strong growth of oil and non-oil imports will be offset by an improvement in the balance of services owing to increased tourism receipts and lower-than-expected payments for technical assistance. The overall balance of payments is expected to show a surplus of about 38 percent of GDP in 2005 due mainly to the receipt of the oil signature bonus on Block 1, which was deposited in the government's national oil account held with the central bank. Gross central bank international reserves (excluding the National Oil Account) remained at around 3.5 months of imports of goods and nonfactor services by end-2005.

8. Fiscal consolidation is progressing as envisaged. At end-September 2005, the domestic primary deficit was slightly lower than programmed, notwithstanding lagging tax collections and delays in payments on account of fishing licenses granted to the European Union. The revenue shortfalls were compensated by large cuts in domestic primary spending, which was significantly below the program ceiling for end-September 2005. Overall, the program fiscal targets for end-2005 are likely to have been met.

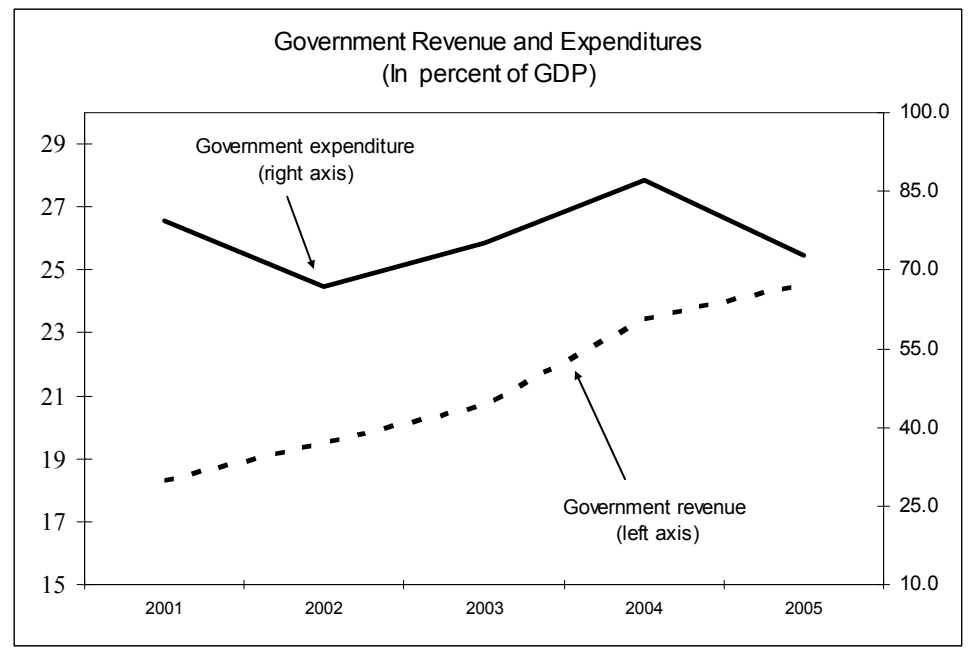


9. Broad money has been growing rapidly in recent months on account of resilient economic growth and an increase in the central bank's net international reserve position. ${ }^{3}$ The latter has mirrored delays in start-up projects in the domestic oil and non-oil sectors (that lowered external payments for technical assistance) and a temporary increase in foreign trade credit to finance a higher oil import bill than originally projected. Pressures on the central bank's reserve position have remained unabated, however, as manifested by a persistent

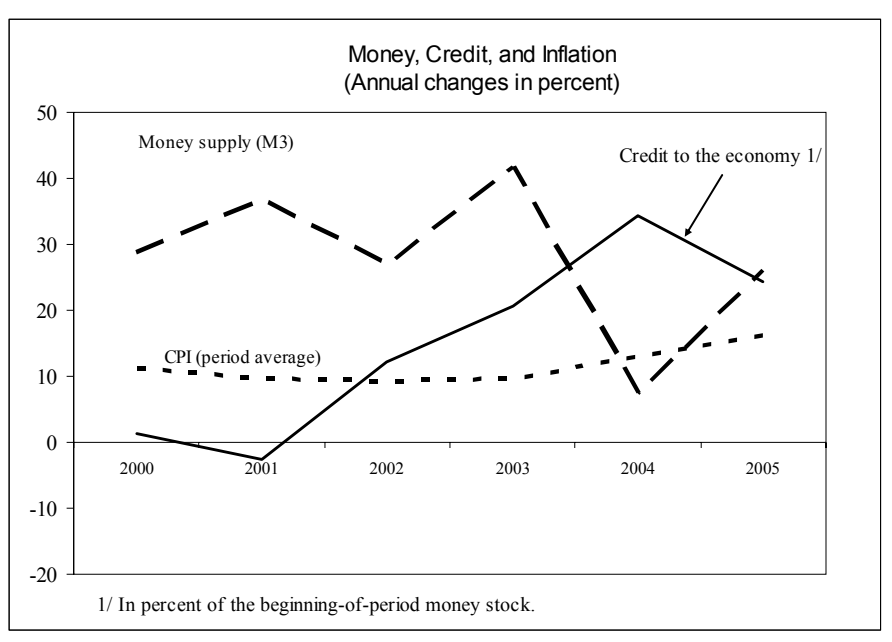
over-subscription of the central bank's foreign exchange auctions.

10. The monetary authorities have reacted to the increase in inflation. The central bank reference rate, which had been unchanged since 2003 at 14.5 percent was increased to 18.2 percent in September 2005 (Figure 2). Banks' reserve requirements were increased from 22 to 24 percent in September, as well. Subsequently, commercial banks increased their lending and deposit interest rates, which, according to preliminary staff estimates, triggered a slow-down in bank credit to the economy.

11. The dobra depreciated against the U.S. dollar in 2005. Most of the dobra depreciation (18.1 percent) took place in the last quarter of 2005, following technical changes to the central bank foreign exchange auction, which were implemented in consultation with the Fund's Monetary and Financial Systems Department (MFD). Changes included: (i) an increased frequency, and (ii) changes in the cut-off exchange rate setting for the auctions. These changes were made to improve the price discovering mechanism of the existing managed float and to ensure, through an increased auction's frequency, that sufficient liquidity is in the market to meet the foreign exchange demand of importers. The real effective exchange rate appreciation which occurred during the first three quarters of 2005 was reversed in the last quarter of 2005 (Figure 3).

\footnotetext{
${ }^{3}$ For comparison purposes with the program's monetary numbers (IMF Country Report No. 05/323, Tables 4 and 5, and Appendix IV, Table I.1), balance sheet data for endSeptember and end-December 2005 have been adjusted in Tables 4, 5, 8 and Appendix I, Table I.1, attached, by removing the balances in the government's National Oil Account (Conta Nacional de Petroleo, CNP) from the definition of central bank net international reserves and net domestic assets. Originally, the CNP was supposed to be held in the government's account with the New York Federal Reserve, not at the central bank.
} 
12. Compliance with banking sector prudential regulation improved in 2005 . The central bank launched an off-site banking supervision reporting system to shore up surveillance over the quality of banking sector's assets, liquidity ratios, capital adequacy, and profitability. A review of on-site examination guidelines is also well advanced, with an onsite surveillance of the leading deposit money bank (Banco Internacional de São Tomé and Principe, BISTP) expected in the first half of 2006. A preliminary assessment by the bank regulators indicates that the BISTP is in sound footing according to off-site prudential regulation indicators.

\section{The implementation of the government's structural reform program moved} forward in 2005. Structural conditionality under the existing PRGF arrangement was implemented on schedule, except for delays in the submission of AML/CFT legislation to the National Assembly. In parallel, consistent with the government's PRSP, the authorities are embarked in a process of institutional development of the oil sector, improved public expenditure and external debt management, public enterprise reform, judicial sector reform, and improved business climate (Table 10).

14. However, achieving full transparency in oil sector related issues has remained challenging. Following the completion of the licensing round of Blocks 2-6 in 2005, the National Petroleum Council, which includes the President of the Republic, the Prime Minister and key cabinet members, issued a communiqué acknowledging allegations about lack of transparency and technical flaws in the awarding of the blocks. Nonetheless, the Council concluded that these flaws were not detrimental to the country's long-term interest and decided to go forward with the process. A subsequent report by the Attorney General's Office of São Tomé and Príncipe has confirmed the existence of irregularities in the process. To date, no decision has been taken by the authorities regarding the Attorney General's findings and the appropriateness of any remedial measures. The staff have emphasized to the authorities the seriousness of these issues and the need to strengthen good governance and transparency in the oil sector.

15. São Tomé and Príncipe is current with its obligations with multilateral creditors and has cleared its arrears with Paris Club creditors as part of a new multilateral debt rescheduling agreement on Cologne terms. The authorities are currently in the process of reconciling debt records with Paris Club creditors, with the expectation of settling any outstanding amounts before end-March 2006. Contacts with non-Paris Club bilateral creditors are also being pursued to start reconciliation of debt records.

\section{Policy Discussions}

16. Policy discussions focused on the need to fundamentally address the economy's macroeconomic imbalances and avoid the fiscal slippages that characterized the stopand-go policy implementation in recent years. The authorities concurred that fiscal consolidation, while preserving an adequate level of pro-poor spending, was critical to sustain macroeconomic stability. They acknowledged that the poor are usually the most affected by high inflation and sluggish economic growth. 
17. The authorities recognized the policy challenges at stake, including the need to manage the prospective large oil revenue against the background of weak institutional and absorptive capacities. In the immediate future, fiscal consolidation would require striking a balance between (i) the population's expectation about the government's ability to increase priority spending in the wake of the oil era and (ii) the available budgetary resources stemming mainly from foreign aid and a gradual use of the oil signature bonuses of 2005-06 in line with the Oil Revenue Management Law. Over the near- and medium-term, a main task would be to develop strong institutions to secure an effective, transparent, and fullyaccountable management of oil revenue. The gradual build up of Permanent Oil Fund for future generations would also be part of this endeavor. As the oil sector is likely to be an enclave, the authorities are actively engaged with development partners to secure a proper execution of the government's PRSP that would strengthen growth in the non-oil economy, reduce poverty, and support the attainment of the MDGs.

\section{The Sãotomean authorities appreciated the advice provided by the Fund} Executive Board in the context of the Article IV consultations, and have been working towards their implementation in the context of the PRGF arrangement (www.imf.org/external/country/STP/index.htm). The authorities particularly welcomed the Board's recommendation during the latest consultation (concluded in March 2004) to adopt policies that are conducive to macroeconomic stability, a reduction in poverty and achieving high growth in the non-oil sector. A key element was the development of strong institutions to ensure a proper management of prospective oil revenue. The Board's advice was instrumental in supporting the adoption of the Oil Revenue Management Law (ORML) in December 2004 and has helped in forging consensus among policymakers toward a successful implementation of the PRGF-supported program to date. Ongoing work at various levels of government seeks to attain an effective development of the supporting institutional framework devised in the ORML. Furthermore, to increase potential output in the non-oil economy, the PRGF-supported program includes a comprehensive structural reform agenda. The authorities also stressed the need of continued technical assistance in the areas of Fund expertise to buttress administrative capacity and policy decision making.

\section{A. Macroeconomic Outlook}

19. The medium-term outlook seems relatively promising in the aftermath of the recent awarding of oil Blocks 2 to 6 , located in the zone jointly operated with Nigeria. In this regard, the baseline medium-term scenario discussed with the authorities illustrates a likely evolution of the economy as the local oil sector develops under conditions of increased macroeconomic stability and continued structural reform. In this context, and in line with past Fund surveillance, the authorities stressed the importance of continued Fund technical assistance in buttressing administrative capacity and helping the deepening of major reforms in revenue, expenditure management, and the monetary and exchange rate policy areas. Domestic investment would increase sharply from 36 percent of GDP in 2003-05 to 42 percent in 2006, and to an average of 54 percent of GDP in 2007-10, largely on account of oil sector investment initiatives. The rise in the investment ratio will require a continuous reliance on foreign savings, although national savings are forecast to rise on account of a 
robust financial position of the government and increased private savings once stability and economic growth become firmly established. Under the baseline scenario, external financing gaps (after revenue measures) would be around 2.5-3.5 percent of GDP per-year between 2007 and 2010, despite the envisaged significant consolidation of the fiscal stance. While the MDRI could liberate about 2 percentage points of GDP, on average, to finance pro-poor spending (see paragraph 31, below), there will be a need for additional external financing of about 1.5 percent of GDP per-year during this period.

\begin{tabular}{|c|c|c|c|c|c|c|c|c|c|c|}
\hline \multicolumn{11}{|c|}{$\begin{array}{l}\text { São Tomé and Príncipe: Baseline Scenario, 2003-2010 } \\
\text { (In percent of GDP) }\end{array}$} \\
\hline & 2003 & 2004 & 2005 & Average & 2006 & 2007 & 2008 & 2009 & 2010 & Average \\
\hline & & & Est. & 2003-05 & Prog. & \multicolumn{4}{|c|}{ Projections } & 2007-10 \\
\hline Gross domestic investment & 36.1 & 35.2 & 36.0 & 35.8 & 42.2 & 51.3 & 55.0 & 55.6 & 53.7 & 53.9 \\
\hline Gross Savings & 36.1 & 35.2 & 36.0 & 35.8 & 42.2 & 51.3 & 55.0 & 55.6 & 53.7 & 53.9 \\
\hline External savings & 22.3 & 20.1 & 33.1 & 25.2 & 28.4 & 29.4 & 31.8 & 30.5 & 29.2 & 30.2 \\
\hline Gross national savings & 13.8 & 15.1 & 3.0 & 10.6 & 13.8 & 21.9 & 23.3 & 25.2 & 24.5 & 23.7 \\
\hline Government & 13.4 & 6.8 & 11.6 & 10.6 & 15.5 & 21.9 & 22.6 & 21.7 & 20.5 & 21.7 \\
\hline Private sector & 0.4 & 8.3 & -8.6 & 0.0 & -1.7 & 0.0 & 0.6 & 3.5 & 4.0 & 2.0 \\
\hline \multicolumn{11}{|l|}{ Memorandum items: } \\
\hline Real GDP (annual percentage change) & 4.0 & 3.8 & 3.8 & 3.9 & 4.5 & 5.5 & 6.5 & 7.0 & 7.5 & 6.6 \\
\hline Consumer prices (percentage change; average) & 9.6 & 12.8 & 16.2 & 12.9 & 14.8 & 11.1 & 8.7 & 7.5 & 6.5 & 8.4 \\
\hline Domestic primary fiscal balance (percent of GDP) & -11.7 & -20.6 & -14.0 & -15.4 & -15.2 & -11.5 & -9.0 & -7.7 & -6.1 & -8.6 \\
\hline
\end{tabular}

20. The authorities broadly agreed with the baseline scenario, but noted that to significantly reduce poverty by $\mathbf{2 0 1 0}$, as targeted in the PRSP, higher economic growth than envisaged was necessary. In particular, the economy would need to grow at an annual average growth rate of 8 percent during 2006-12, compared with a 6 percent average growth rate in the baseline scenario, to achieve the MDGs (Table 11). The staff agreed with the authorities that achieving the MDGs would require a substantially higher donor support than envisaged in the baseline scenario. A gradual and transparent use of the oil signature bonuses - consistent with the Oil Revenue Management Law and as included in the baseline scenario - could, in part, substitute for the ongoing decline in foreign assistance and traditional short-term borrowing to finance the budget. However, these oil resources would not be enough to fill the resource gap.

\section{B. Fiscal Policy}

21. The authorities stressed their determination to sustain the process of fiscal consolidation that started in 2005 . They underlined the need to increase tax revenue, reduce wasteful expenditure, improve the composition of government spending by increasing the share of capital and pro-poor expenditure in line with the government's PRSP, and seek to eliminate government recourse to central bank financing.

22. The authorities are implementing measures to strengthen revenue collection over the short and medium term (Box 2). A public campaign is currently underway stressing the government's decision to improve tax compliance and reduce tax arrears, 
particularly in the case of direct taxes. The authorities are also working with the Millennium Challenge Corporation to launch an overhaul of the customs administration system during 2006. Over the medium term, the emphasis would be to review the existing personal and corporate income tax codes; draft legislation of these codes is currently under review by the National Assembly.

\section{Box 2. São Tomé and Príncipe: Revenue Measures in the Authorities' Medium-Term Fiscal Plan}

\section{Measures implemented in 2005 Program}

- Increase dobra-denominated reference prices used in assessing excise duties on a core group of nonfood imports.

- Increase excise tax rate on the provision of services.

- Terminate concession on excise duty levied on domestically produced beer.

- Increase tax base for assessing urban property taxes.

- Keep retail petroleum prices in line with world market prices. Retain ad-valorem surcharge on gasoline and on gasoil.

\section{Measures included in 2006 Program}

- Consolidate tax measures introduced in 2005.

- Expedite settlement of corporate and individual income tax arrears.

- Reformulate existing customs procedures to increase tax base and collections.

- Strengthen customs services through better surveillance and increased number of random inspections of importers' warehouses.

\section{Medium-term tax measures}

- Issue new codes for personal and corporate income taxation.

23. On the expenditure side, the government's objective is to change the composition of government outlays in favor of pro-poor expenditure. This implies implementing a number of investment projects in agriculture and fisheries, which are in line with the PRSP and the government's efforts to address the MDGs. Specifically, the spending envelope for capital expenditure financed by the treasury and HIPC-related social expenditure will increase from 6.2 percent of GDP in 2005 to 9.1 percent in 2006, and 11.3 percent of GDP in 2007. Current expenditure (excluding budgetary allocations for the holding of parliamentary, regional, and presidential elections in 2006) will decline gradually as a share of GDP over the medium term. A key element will be the wage bill, which is projected to increase above the inflation rate, but to gradually decline in terms of GDP over the medium term. This objective will be achieved by pursuing a strict management of the payroll through the activation of the civil servants' register that is being developed in consultation with the UNDP.

\section{The authorities' medium-term fiscal plan embraces a number of policies aimed} at improving expenditure management. These measures include the implementation of a fully integrated, computerized budget and public expenditure management system (SIGFE), 
and the launching of a reform of the auditing practices of the financial operations of the general government and public enterprises by the Inspectorate-General of Finance.

\section{Monetary and Exchange Rate Policies}

25. The authorities discussed with staff the factors contributing to the acceleration of inflation in 2005. The authorities acknowledged the role of monetary variables in explaining inflation, but noted that the volatility of monetary aggregates reflected the impact of intermittent inflows of foreign aid and the monetization of oil signature bonuses. In this regard, the authorities argued that inflation in 2005 reflected mainly supply shocks stemming from increases in international oil prices that were passed-through to domestic consumers and the inflationary impact stemming from food shortages due to a poor agricultural harvest and temporary breakdowns in the commodity import distribution channels into the islands. The staff discussed with the authorities some preliminary econometric analysis showing that inflation in São Tomé and Príncipe is positively correlated with both the depreciation of the exchange rate and broad money growth. The staff also stressed the transitory nature of some price increases driven by supply shocks. The authorities welcomed the staff's analysis and restated their support for the development of indirect monetary instruments, an area in which the central bank is receiving technical assistance from the Fund. ${ }^{4}$

26. The staff agreed with the authorities that monetary policy continues to play a key role in achieving the program's inflation target, with base money as the nominal anchor. The central bank will use a judicious mix of monetary instruments, primarily foreign exchange sales and the issuance of central bank certificates of deposit (CDs), to sterilize excess liquidity. In this context, it was agreed that base money will be closely monitored in the program. The authorities also reiterated the view that the central bank reference rate should be kept above the inflation rate and, if needed, banks' reserve requirements will be used until indirect instruments of monetary policy are further developed. Coordination of fiscal and monetary policies will also be enhanced in 2006 through regular meetings between the BCSTP and the Ministry of Planning and Finance aimed at forecasting liquidity constraints.

27. The authorities and the staff concurred that the existing managed float exchange rate system has served the country well so far, as it helps cushion exogenous shocks. The temporary appreciation of the real effective exchange rate during the first three quarters of 2005 - which fully reverted to its end-December 2004 level by end-2005- does not seem to have had an adverse effect on non-oil export activities, international reserves remain broadly adequate, and the exchange rate does not appear to be currently misaligned. The cumulative depreciation of the real effective exchange rate between mid-2001 and end-December 2005 has been about 15 percent. Nevertheless, cocoa export volumes have reflected mainly supply conditions and are mostly directed to niche markets in Western Europe. Tourism, which has benefited from a trend increase in foreign direct investment, is a high value added sector

\footnotetext{
${ }^{4}$ See Chapter III in the accompanying Selected Issues Paper.
} 
attracting a selected group of wealthy outdoor adventure and eco-tourists. Also, import coverage of reserves, excluding the National Oil Account, is above three and a half months of imports of goods and services; reserves are equivalent to about 150 percent of base money, on average. ${ }^{5}$ The monetary authorities expressed their appreciation of the Fund's advice on exchange rate policy and its timely provision of technical assistance. The staff commended the authorities on their commitment to improve the foreign exchange market and the work done so far in this area.

\section{Banking Sector}

28. The authorities reiterated their commitment to enhancing the central bank's banking sector supervisory capacity, while securing an effective implementation of the reforms introduced in 2005 (Box 3). Decisive action regarding weak banks, including recapitalization or closure of affected banks, when appropriate, would be critical to foster the development of an efficient and sound domestic banking system.

\section{Box 3. São Tomé and Príncipe: Financial Sector Reform-Action Plan for 2006}

- Strict implementation of new procedures for licensing financial institutions.

- Regular compilation of banks' consolidated reporting statistics.

- Implementation of scheduled on-site banks' examinations.

- Issuance of new guidelines on banks' capital requirements, including elements of Basel II recommended benchmarks.

- Development of a "credit bureau" to assess overall financial sector's credit risk.

\section{E. External Sector Policies}

29. Discussions on external sector policies focused on ways to diversify exports of goods and services, while creating employment in the non-oil economy (Box 4). The authorities indicated that actions in all these areas were proceeding according to plan. Also, the authorities informed the staff that São Tomé and Príncipe applied for membership at the World Trade Organization (WTO) in January 2005. The Working Party on São Tomé and Príncipe's accession will meet following the authorities' submission of the required Memorandum on the Foreign Trade Regime.

\footnotetext{
${ }^{5}$ Information collected by the central bank indicates that the spread between the official and the parallel exchange rates of the dobra was 2 percent at end-2005. However, there are neither consistent time series data on parallel exchange rates, nor independent sources of information to confirm the quality of the central bank data.
} 


\section{Box 4. São Tomé and Príncipe: External Sector Policies—Status Report}

- Draft legislation is currently with the National Assembly to eliminate tariff exemptions granted to public institutions on imports of raw materials and capital equipment, and to political parties on imports of goods and vehicles during the pre-election campaign.

- A newly-completed Diagnostic Trade Integration Study (DTIS) recommends a number of measures to expand and diversify exports. Key measures included in the 2006 Fund-supported program are (i) the approval of a new investment code and new codes for personal and corporate income taxation, (ii) the simplification of customs procedures, and (iii) the preparation of a strategy for the electricity sector and the restructuring of the public enterprise administering seaports (ENAPORT).

- $\quad$ São Tomé and Príncipe has an observer status in CEMAC and is participating in preparations for upcoming Economic Partnership Agreement (EPA) negotiations with EU.

30. The mission agreed with the authorities that São Tomé and Príncipe's trade regime is relatively open. The tariff regime schedule was adjusted in mid-2002, shifting some agricultural and industrial goods among the three tiers of 5 percent, 10 percent, and 20 percent. $^{6}$ The only remaining non-tariff barrier protecting the domestic telecommunications company from competition expired on December 31, 2005.

\section{São Tomé and Príncipe no longer maintains exchange restrictions under the} transitional arrangements of Article XIV of the Articles of Agreement. The authorities have expressed their intentions to accept the obligations of Article VIII, Sections 2 (a), 3 and 4 in the near future. In December 2005, a joint LEG/MFD mission visited São Tomé to conduct a review of the exchange system. While staff found evidence that suggests that São Tomé and Príncipe may maintain a number of restrictions subject to Fund approval, staff and the authorities are still in the process of finalizing the review of the exchange system, and staff intends to present the findings and recommendations at the time of the second review under the PRGF arrangement.

\section{São Tomé and Príncipe's public debt will remain high over the medium term}

(Appendix V). Even after assuming debt rescheduling from the Paris Club in 2005, and further debt relief after reaching HIPC completion point in 2006, the NPV of debt-to-export ratio is only projected to fall from 500 percent at end-2005 to 246 percent by end-2011. However, preliminary staff calculations suggests that the country would benefit from a significant reduction in debt service under the MDRI once the HIPC completion point is reached, hopefully in mid-2006. ${ }^{7}$

\footnotetext{
${ }^{6}$ Basic goods are in the 5 percent band, while luxury goods are taxed at the 20 percent rate. All other goods are taxed at the 10 percent rate. There are surcharges, notably for vehicles, fuel, tobacco, and alcohol.

${ }^{7}$ Tables 1-7 do not include the impact of the MDRI.
} 


\section{F. Structural Reform and Governance Issues}

33. The authorities are committed to accelerate structural reform with a view to foster private sector development and increase the growth potential of the economy. In line with the government's PRSP, the authorities intend to revisit property rights for land tenure, deepen the process of judicial reform, implement measures to improve the country's business climate, and push forward with public enterprise reform and restructuring.

34. The authorities also intend to assess ways to maximize the spillover effects of the development of the oil sector on the non-oil economy. To this end, the National Petroleum Agency (ANP) is developing a master document to identify local businesses and/or joint ventures based in the islands that could become suppliers of intermediate goods or services to the oil exploration industry. The expectation is to safeguard the efficiency of spending in the oil sector, while increasing the local content of intermediate demand and other income multiplier effects in São Tomé and Príncipe.

\begin{tabular}{|c|c|c|c|c|c|c|c|c|}
\hline \multicolumn{9}{|c|}{$\begin{array}{l}\text { São Tomé and Príncipe: DSA Baseline and MDRI Scenarios 1/ } \\
\text { (In percent) }\end{array}$} \\
\hline & \multirow{2}{*}{$\frac{\text { Est. }}{2005}$} & \multicolumn{7}{|c|}{ Projections } \\
\hline & & 2006 & 2007 & 2008 & 2009 & 2012 & 2015 & 2025 \\
\hline \multirow{4}{*}{$\begin{array}{l}\text { Before MDRI } \\
\text { After MDRI }\end{array}$} & \multicolumn{8}{|c|}{ NPV of debt-to-GDP ratio } \\
\hline & 153 & 110 & 106 & 102 & 97 & 22 & 7 & 10 \\
\hline & $\ldots$ & 61 & 62 & 61 & 60 & 14 & 5 & 7 \\
\hline & \multicolumn{8}{|c|}{ NPV of debt-to-exports ratio } \\
\hline Before MDRI & 500 & 315 & 302 & 289 & 282 & 46 & 7 & 20 \\
\hline \multirow[t]{2}{*}{ After MDRI } & $\ldots$ & 176 & 176 & 174 & 175 & 30 & 5 & 14 \\
\hline & \multicolumn{8}{|c|}{ Debt service-to-export ratio 2/ } \\
\hline Before MDRI & 56.0 & 19.1 & 12.4 & 11.4 & 10.6 & 2.4 & 0.4 & 2.1 \\
\hline \multirow[t]{2}{*}{ After MDRI } & $\ldots$ & 9.9 & 6.7 & 6.5 & 6.0 & 1.8 & 0.3 & 1.3 \\
\hline & \multicolumn{8}{|c|}{ Debt service-to-GDP ratio } \\
\hline Before MDRI & 17.1 & 6.7 & 4.4 & 4.0 & 3.6 & 1.2 & 0.4 & 1.1 \\
\hline After MDRI & $\cdot$ & 3.5 & 2.4 & 2.3 & 2.1 & 0.8 & 0.3 & 0.7 \\
\hline
\end{tabular}


35. In regards to governance issues, the authorities re-affirmed the government's commitment to zero tolerance for acts of corruption. The authorities stressed the need to observe the letter and spirit of the Oil Revenue Management Law (ORML), especially regarding the auditing and oversight of oil revenue inflows and outflows, the financing of the fiscal budget, and the publication and dissemination of relevant information. Key actions in these areas include ongoing technical work at the National Assembly on draft legislation

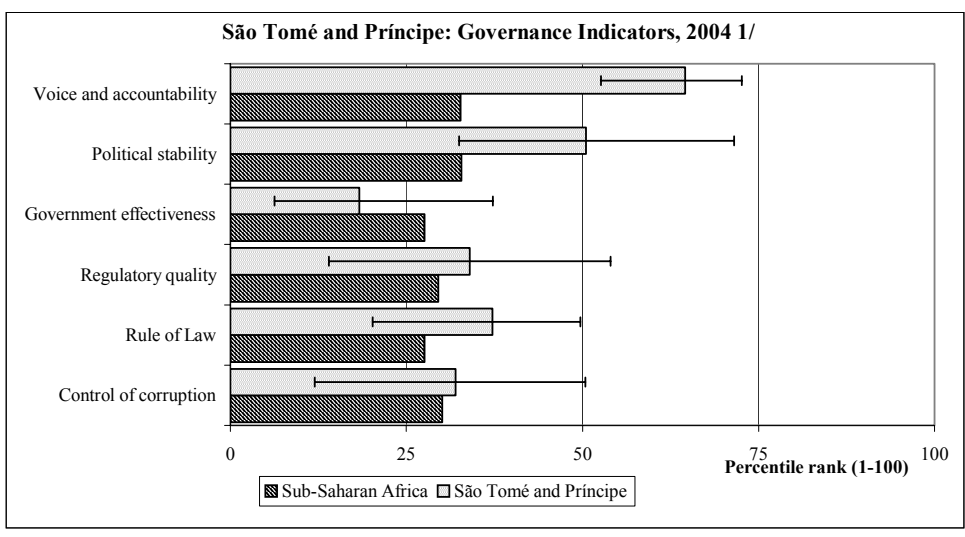

Source: World Bank Governance Indicators $1 /$ Higher rank reflects better governance. establishing the Petroleum Oversight Commission, the Public Registration and Information Office, and a special law regulating the civil, criminal, and administrative responsibilities to handle violations of the ORML. The authorities concurred with the staff on the seriousness of the allegations regarding the lack of transparency and technical flaws in the licensing round of Blocks 2-6. In line with the Treaty between São Tomé and Príncipe and Nigeria on the Joint Development Zone, the authorities intend to raise the issue at the forthcoming Joint Ministerial Committee meeting, scheduled for early February 2006.

\section{G. Poverty Reduction Strategy}

\section{The authorities informed the mission that the first PRSP annual report is} currently under preparation by the Ministry of Planning and Finance. The report should provide a candid description of the PRSP's implementation setbacks to date. Nevertheless, the report will recommend giving a strong boost to the strategy during the next three years, through an effective implementation of the Priority Actions Program (PAP) that was discussed with donors at the recent roundtable meeting in Brussels (December 6, 2005). The PAP identifies financial needs of US\$170 million for 2006-08, of which some U\$70 million were pledged at the Brussels roundtable, marginally above previously existing commitments.

\section{Steady progress has been accomplished towards the implementation of all} trigger points laid out in the HIPC decision point document (Table 12). The sectoral strategies for health and education have already been developed and costed. Public expenditure management is progressing, including a close monitoring over the use of HIPC funds, better programming and execution of foreign-financed capital expenditure, and the ongoing development of a computerized public expenditure management system. In addition, the government established the Auditor General's Office in mid-2003 and issued the Oil Revenue Management Law in December 2004. 


\section{H. Statistical Issues}

38. The authorities are committed to improve the coverage, timeliness, and periodicity of basic macroeconomic statistics with a view to better monitor economic developments and increase the transparency of financial operations in various sectors of the economy. ${ }^{8}$ São Tomé and Príncipe's core economic data are generally made available on a timely basis for program monitoring and surveillance.

São Tomé and Príncipe: Priority Actions Program (PAP), 2006-2008

(In millions of dollars)

\begin{tabular}{cc}
\hline Economic Areas & Financing \\
Needs \\
\hline I. Reform of Public Institutions & $\mathbf{1 9 . 8}$ \\
of which: Economic and financial governance & 9.5 \\
Judicial reform & 4.5 \\
II. Accelerated and Redistributive Growth & $\mathbf{5 9 . 0}$ \\
of which: Roads and transportation & 41.4 \\
Energy sector development & 5.5 \\
Development of agriculture, livestock farming, & \\
forestry and fishing & 4.4 \\
III. Fostering Income Opportunites for Poor & $\mathbf{4 3 . 7}$ \\
of which: Secure water supply systems and & \\
other rural infrastructure & 22.1 \\
Increase yields in farming and fisheries & 11.5 \\
Guarantee food security & 2.5 \\
IV. Development of Human Resources & $\mathbf{4 5 . 6}$ \\
of which: Health & 19.2 \\
Education & 23.8 \\
V. Monitoring of PRSP & $\mathbf{1 . 7}$ \\
Total PAP & $\mathbf{1 6 9 . 8}$ \\
\hline Sour Satomean athorities.
\end{tabular}

Source: Saotomean authorities.

\footnotetext{
${ }^{8}$ Outstanding statistical issues are reviewed in Appendix VI.
} 


\section{Program FOR 2006}

39. The program for 2006 - detailed in the attached Memorandum of Economic and Financial Polices - is consistent with the authorities' medium-term policy framework and focuses on consolidating macroeconomic stability and structural reforms (Appendix I). Real GDP growth is projected to increase to 4.5 percent, while inflation is expected to decline to 13 percent in 2006. The external current account deficit (excluding transfers) is expected to remain large (at about 60 percent of GDP) against the background of a high oil import bill and large investment-related imports. Gross international reserves (excluding the National Oil Account) will remain above three and a half months of imports of goods and services.

40. Fiscal consolidation will continue. However, the domestic primary deficit will increase by one percentage point of GDP to 15 percent in 2006, on account of increased priority spending consistent with the government's PRSP (MEFP, $\mid 8$ ). Net central bank credit to the government will be negative to allow adequate financing to the private sector.

41. The government's efforts to increase tax revenue will be maintained (MEFP, $\lceil 9$ ). The program's revenue targets will be supported by (i) the full-year effect of the tax measures introduced in late 2005, (ii) increases in tax-related receipts on oil, (iii) the collection of tax arrears on corporate and individual income taxes, and (iv) the implementation of the reform of the customs administration to facilitate trade and align customs' inspection and verification systems with best international practices.

42. During 2006, the share of pro-poor spending in the government's overall expenditure envelope will increase in tandem with an upgrading of public expenditure management systems (MEFP, $\llbracket 10-13$ ). Efforts to contain the growth of current expenditure, particularly the wage bill, will continue. The latter objective will be addressed through a strict management of the payroll using the civil servants' register that is being developed in consultation with the UNDP.

43. Monetary policy will continue to be prudent in the context of a flexible exchange rate regime (MEFP, $\mid 14-18$ ). The central bank will continue to implement a judicious mix of sterilization instruments to mop up excess liquidity. The staff welcome the intention of the monetary authorities to continue strengthening banking supervision.

44. In the structural front, the focus will be to reform key public enterprises to further improve the private sector environment (MEFP, -24-26). A comprehensive strategy has been launched to improve the finances of EMAE and safeguard an adequate supply of electricity over the medium term. Key measures in this regard include an increase in utilities tariffs to cover costs of electricity production and the introduction of a system of prepayment through electricity metering keypads to address customers' arrears. Feasibility studies of the public enterprises administering the airports (ENASA) and seaports (ENAPORT) will be prepared with World Bank financial support. A key objective would be to assess the scope for private sector investment in the sector. Also, to improve the business climate, the government will (i) repeal legislation unduly increasing the number of days 
needed to establish a business in São Tomé and Príncipe, and (ii) develop tribunals of arbitration for business litigation. In addition, approval of critical legislation - including a new tax procedural code and new tax codes for personal and corporate income taxation-by the National Assembly is targeted for 2006. Finally, in support of full transparency in oil revenue management, the authorities have recently submitted a formal request to the Joint Development Agency (JDA), recommending the prompt publication of the PSA on Block 1 that was signed between the JDA and the selected oil operator. All other regulations governing the oil sector are already public information.

\section{Program Monitoring, Risks, ANd SAFeguards Assessment}

45. The second review under the PRGF arrangement is expected to be completed no later than end-June 2006, and will make available the third disbursement (Tables 13 and 14), subject to the observance of the quantitative performance criteria set for endDecember 2005 and the definitions set out in the corresponding technical memorandum of understanding (IMF Country Report No. 05/323, Appendix IV, Attachments II and II). The fourth disbursement which will become available upon completion of the third review scheduled for November 2006, will be subject to the end-June 2006 performance criteria specified in Tables I.1 and I.2 in the MEFP (Appendix I, Attachment I). The attached technical memorandum of understanding (TMU; Appendix I, Attachment II) details the design of the program. Minor changes were made to the TMU to clarify a few definitions.

46. The program is subject to a number of risks. First, the process of fiscal consolidation may weaken in the wake of legislative and presidential elections scheduled for late-March and the second half of 2006, respectively, and intense social pressures for a prompt delivery of priority spending before oil production starts. Second, inaction by the authorities to follow suit on the allegations of irregularities in the bidding process of Blocks 2-6 could hamper the credibility of the country, thus hindering the development of the oil sector. Third, possible shortfalls in foreign assistance triggered by the prospective oil boom could exacerbate the government's budget constraint in the short run.

47. The monetary authorities are continuing to address the weaknesses identified in the Fund' safeguards assessment conducted in 2004 (MEFP, -25). The BCSTP has published the external audit of its 2003 and 2004 financial statements, and will publish its audited financial statements on a regular basis from now on.

\section{StAFF APpraisal}

48. São Tomé and Príncipe needs to continue to address macroeconomic imbalances and set the conditions for sustained economic growth and poverty alleviation. Over the medium term, a main challenge would be to develop strong institutions to secure a transparent management of oil revenue. As the oil sector is likely to be an enclave, the authorities need to secure a proper execution of the government's Poverty Reduction Strategy Paper that would strengthen growth in the non-oil economy, reduce poverty, and support the attainment of the MDGs. Lasting and enhanced donors' support to finance the PRSP would be vital in this regard. 
49. The staff supports the authorities' decision to sustain the process of fiscal consolidation that started in $\mathbf{2 0 0 5}$, while safeguarding an adequate level of pro-poor spending. The staff concurs on the need to increase tax revenue, reduce wasteful expenditure, and improve the composition of government spending by increasing the share of pro-poor expenditure in line with the PRSP. Fiscal consolidation would also be enhanced by the implementation of a fully integrated, computerized budget and public expenditure management system (SIGFE), and the reform of the auditing practices of the general government's and public enterprises' financial operations.

50. The staff concurs that the overall stance of financial policies remains broadly adequate, with monetary policy continuing to play a key role in achieving the government's inflation target and safeguarding the central bank's international reserve position. To control liquidity, the staff encourages the authorities to continue to make judicious checks between foreign exchange sales and the issuance of liquidity absorbing instruments, such as central bank certificates of deposits. The staff supports the authorities' commitment to keep the central bank reference rate above the inflation rate and to improve the coordination of fiscal and monetary policies to better forecast liquidity. Recent progress in bank supervision is welcome.

51. In the staff's view, São Tomé and Príncipe's managed floating exchange rate system remains appropriate. It allows the monetary authorities to address the volatility of external inflows and cushion exogenous shocks. The staff commends the BCSTP for improving its foreign exchange auction system to strengthen the price discovering mechanism of the existing managed float rate.

52. Helped by the tariff reforms in 2002, São Tomé and Príncipe has a relatively open trade regime. The staff commends the authorities for the termination, in endDecember 2005, of the only remaining nontariff barrier protecting the domestic telecommunications company from competition.

53. The staff commends the authorities on their efforts to enhance the central bank's banking sector supervisory capacity. Critical in this regard would be an effective implementation of the measures introduced in 2005 regarding the licensing of new banks and the development of on-site and off-site prudential regulation indicators. Decisive action regarding weak banks, including recapitalization or closure of affected institutions, when appropriate, would be also important to foster the development of an efficient and sound domestic banking system.

54. The staff supports the authorities' commitments to accelerate structural reform and achieve full transparency in oil revenue management. A key goal for 2006 will be to implement the action plan to improve the finances of EMAE and complete the feasibility studies of ENASA and ENAPORT. Also essential would be to improve the business climate, including the elimination of administrative barriers to start-up businesses, the establishing of tribunals of arbitration, and the approval of new codes for personal and corporate income taxation. Publication of the PSAs signed between the JDA and the selected oil operators 
would be an important milestone toward securing accountability and good governance in the nascent oil sector.

55. The staff welcomes the authorities' commitment to improve the coverage, timeliness, and periodicity of basic macroeconomic statistics.

56. The staff recommends the completion of the first review under the PRGF arrangement. All the quantitative and structural performance criteria set for endSeptember 2005 under the current PRGF arrangement were met.

57. The staff welcomes the authorities' stated interest in accepting the obligations of Article VIII, Sections 2 (a), 3 and 4 and the progress made in the review of the exchange system.

58. The staff welcomes the authorities' intention to publish the staff report, the letter of intent, and the MEFP. It is recommended that the next Article IV consultation with São Tomé and Príncipe take place within 24 months subject to the provisions of the decision on consultation cycles in program countries. 
Figure 1. São Tomé and Príncipe: Real Economy

Inflation Rate, January 2004-December 2005 (In percent, 12-month change)

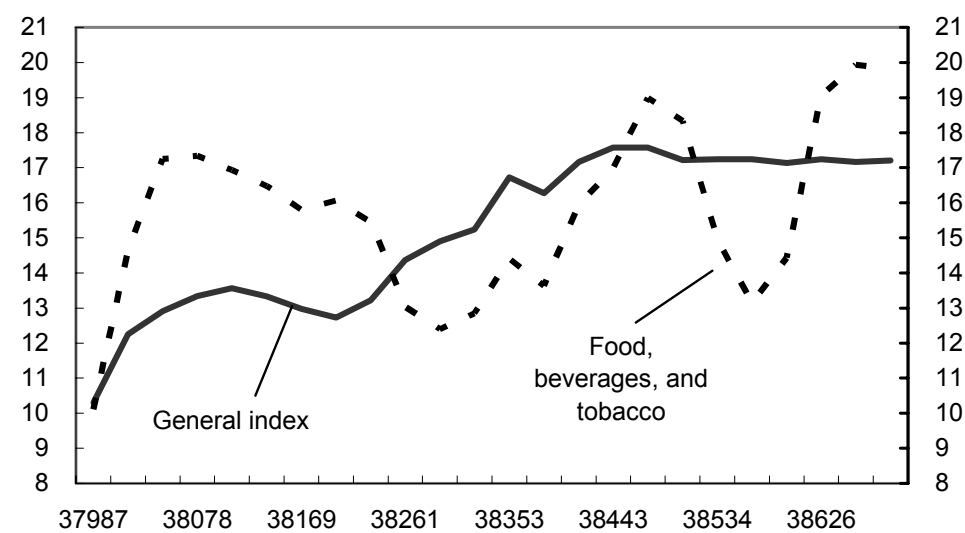

Electricity Consumption, December 2003-August 2005 (In Kilowatt-hours; 12-month accumulated)

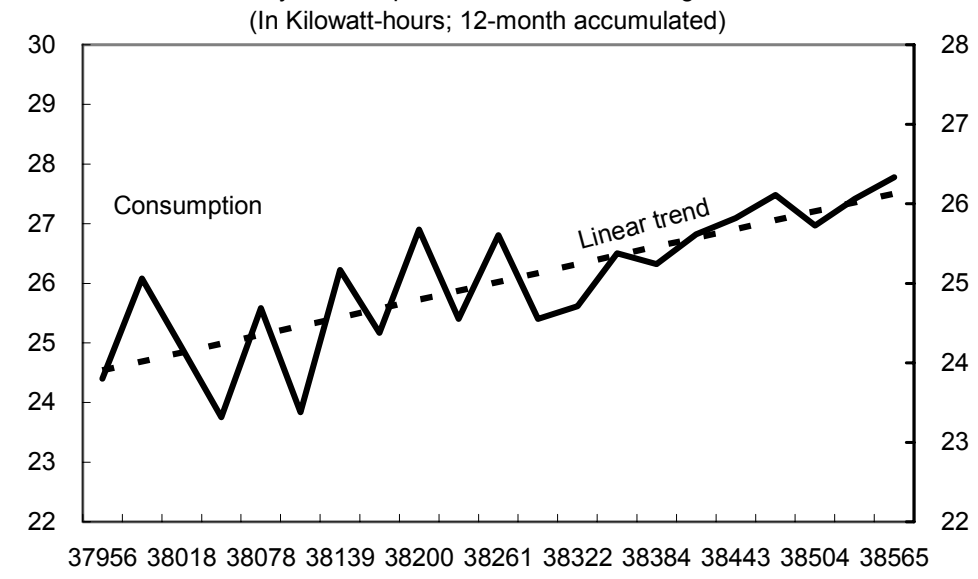

Commodity Imports and Export, January 2003-October 2005 (In millions of U.S. dollars; 12-month accumulated)

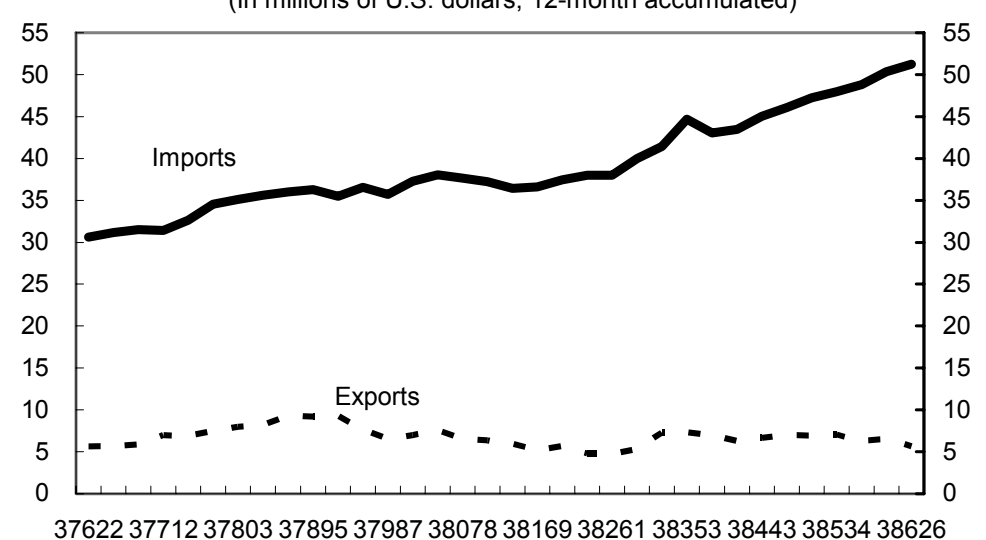

Terms of Trade, 2000Q1-2005Q2 1/

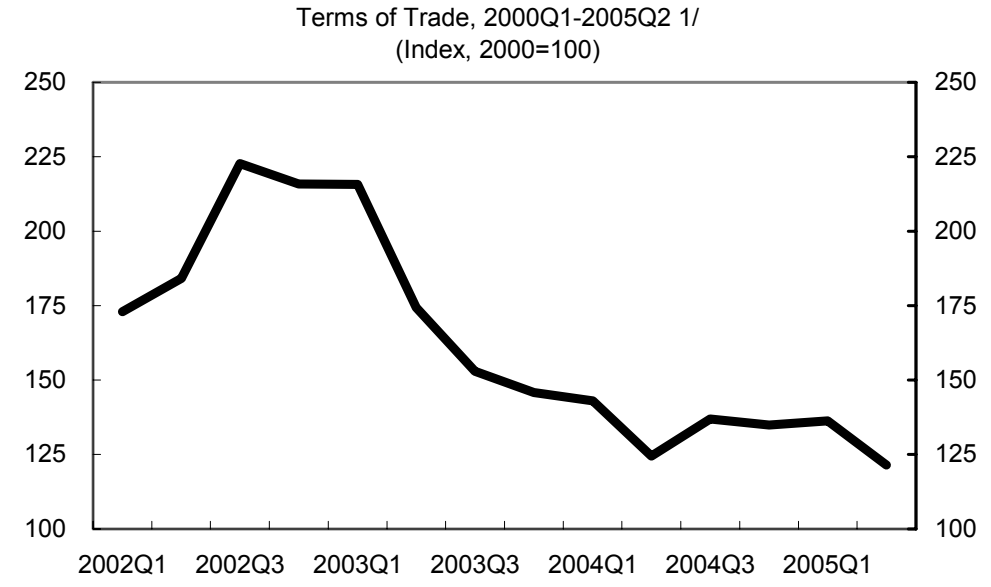

Sources: São Tomé and Príncipe authorities; and WEO, World Economic Outlook.

1/ Defined as export prices divided by import prices. 
Figure 2. São Tomé and Príncipe: Monetary Indicators

Inflation and Interest Rates, January 2002-December 2005 (In percent, 12-month)

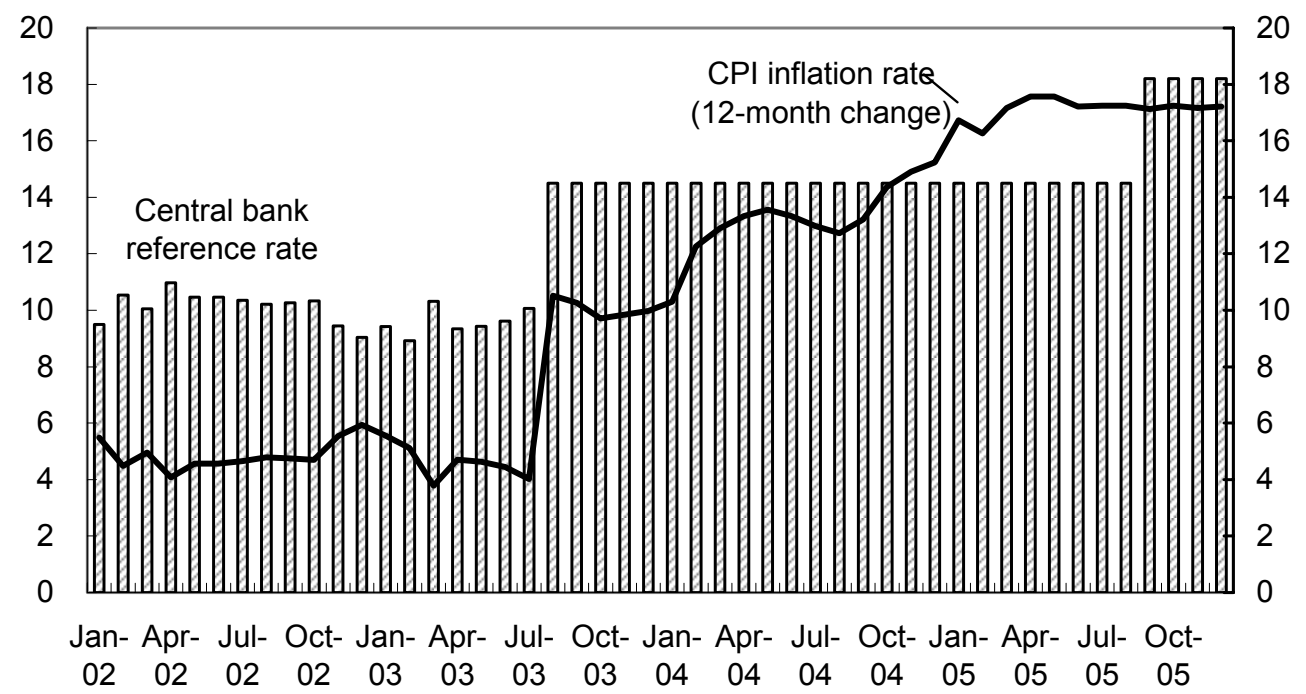

International Reserves and Exchange Rate

December 2002-November 2005

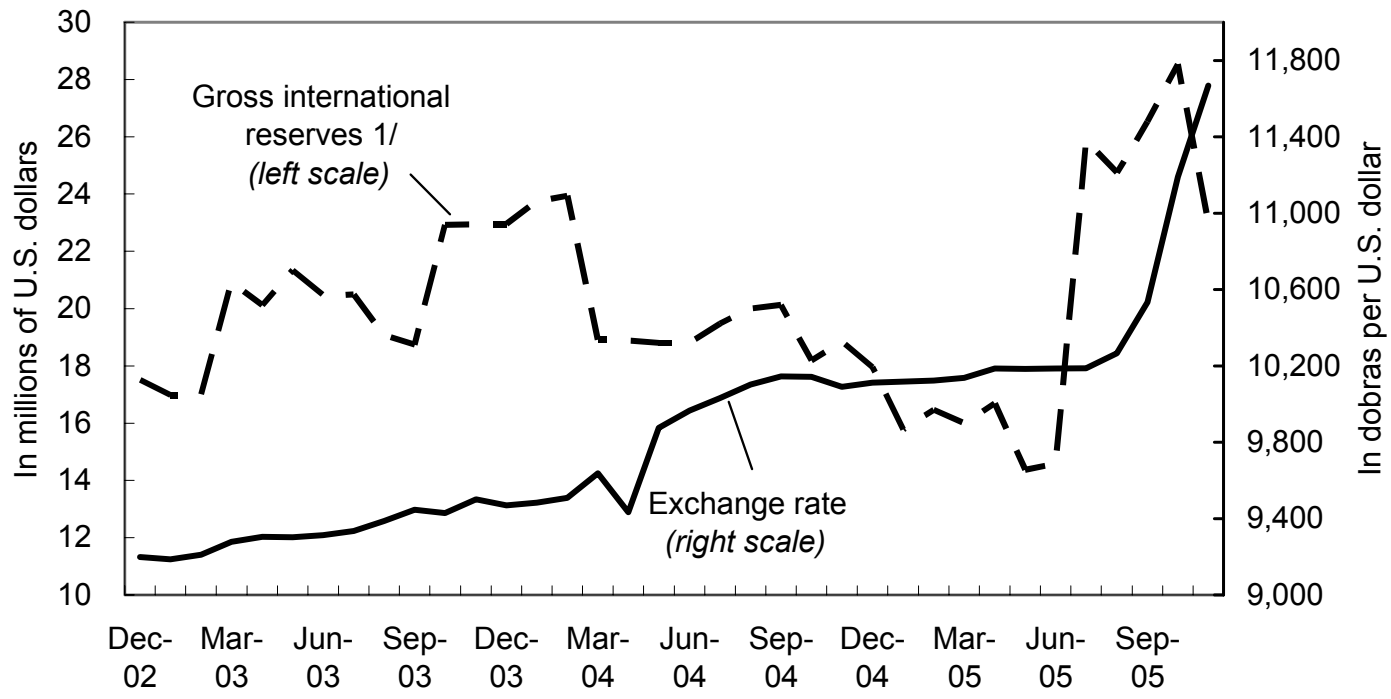

Source: Central Bank of São Tomé and Príncipe.

1/ Excluding the National Oil Account and guarantee deposits by prospective financial institutions pending operating licenses. 
Figure 3. São Tomé and Príncipe: Effective Exchange Rates, January 2000-October 2005 1/

(Index; $2000=100)$

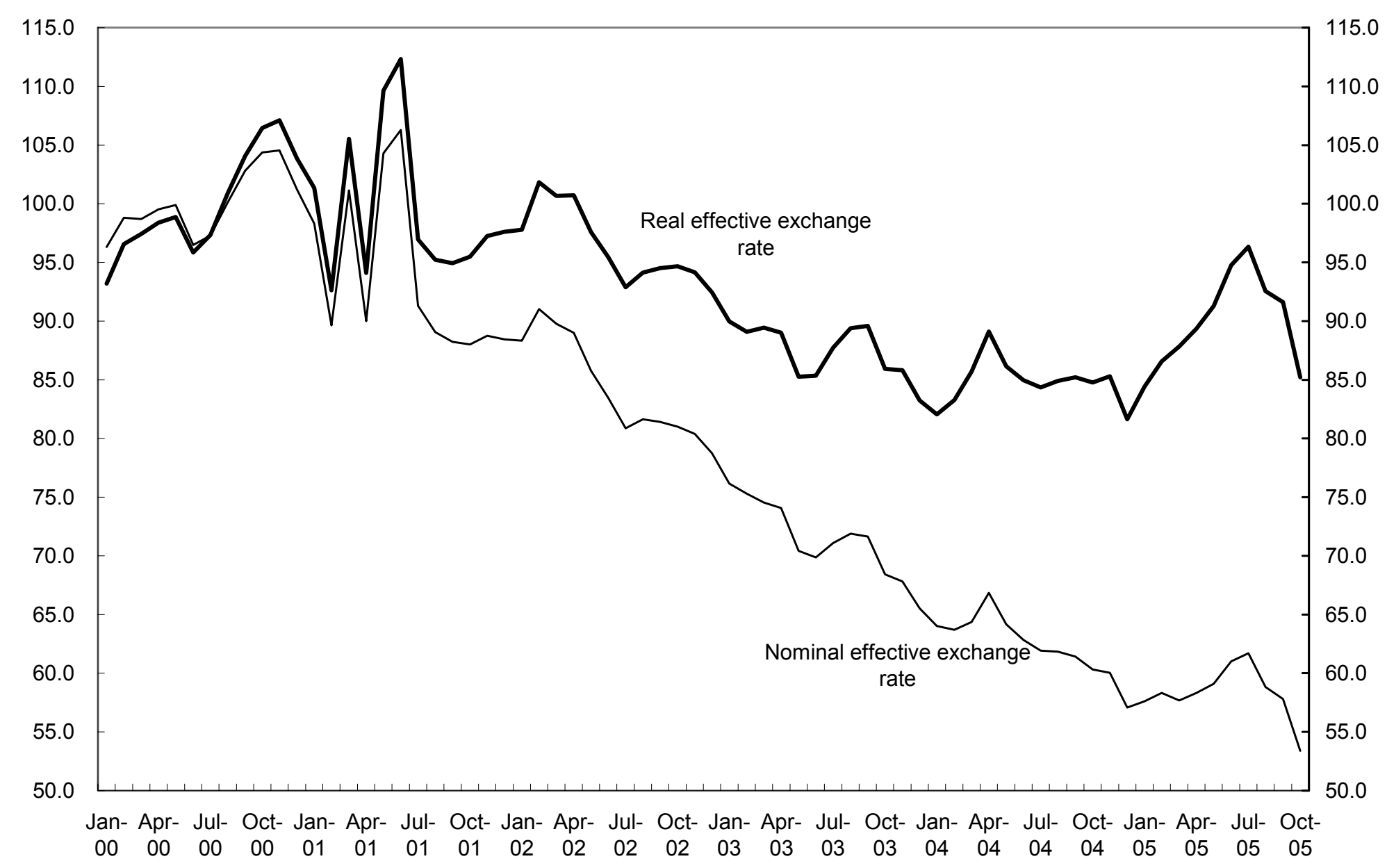

Source: INS, Information Notice System.

1/ An increase in the index corresponds to an appreciation of the effective exchange rate. 
Table 1. São Tomé and Príncipe: Selected Economic Indicators, 2003-10

\begin{tabular}{|c|c|c|c|c|c|c|c|c|c|}
\hline & \multirow[t]{2}{*}{2003} & \multirow[t]{2}{*}{2004} & \multicolumn{2}{|c|}{2005} & \multirow{2}{*}{$\begin{array}{l}2006 \\
\text { Prog. }\end{array}$} & 2007 & 2008 & \multirow{2}{*}{2009} & \multirow[t]{2}{*}{2010} \\
\hline & & & CR $05 / 323$ & Est. & & & Pro & & \\
\hline & \multicolumn{9}{|c|}{ (Annual percentage changes, unless otherwise specified) } \\
\hline \multicolumn{10}{|l|}{ National income and prices } \\
\hline GDP at constant prices & 4.0 & 3.8 & 3.2 & 3.8 & 4.5 & 5.5 & 6.5 & 7.0 & 7.5 \\
\hline Consumer prices (percentage change; end of period) & 10.2 & 15.2 & 15.0 & 17.0 & 13.0 & 9.4 & 8.0 & 7.0 & 6.0 \\
\hline Consumer prices (percentage change; average) & 9.6 & 12.8 & 15.1 & 16.2 & 14.8 & 11.1 & 8.7 & 7.5 & 6.5 \\
\hline \multicolumn{10}{|l|}{ External trade } \\
\hline Exports, f.o.b. & 29.4 & -47.0 & 6.0 & 8.6 & 5.3 & 5.0 & 2.4 & 2.3 & 6.8 \\
\hline Of which: cocoa & 34.9 & -48.8 & 7.9 & 8.7 & 2.1 & 4.7 & -1.1 & -0.8 & -0.9 \\
\hline Imports, c.i.f. & 18.1 & 7.1 & 4.6 & 17.0 & 4.5 & 12.3 & 9.5 & 8.3 & 8.5 \\
\hline Exchange rate (in dobras per U.S. dollar; end of period) & 9,456 & 10,104 & 11,396 & 11,930 & $\ldots$ & $\ldots$ & $\ldots$ & $\ldots$ & $\ldots$ \\
\hline Real effective exchange rate (depreciation -) 1/ & -8.7 & -2.7 & $\ldots$ & 4.4 & $\ldots$ & $\ldots$ & $\ldots$ & $\ldots$ & $\ldots$ \\
\hline Terms of trade & -10.1 & -18.8 & 0.2 & -5.2 & -4.0 & 1.7 & -0.6 & -0.8 & -1.1 \\
\hline \multicolumn{10}{|l|}{ Money and credit (end of period) } \\
\hline Net foreign assets $2 / 3 /$ & 30.8 & -9.8 & 8.7 & 125.5 & 107.7 & 29.3 & 17.2 & 19.2 & 16.8 \\
\hline Net domestic assets $2 / 3 /$ & 11.0 & 17.1 & 3.6 & -99.4 & -91.6 & -13.9 & -4.0 & -4.9 & -2.4 \\
\hline Of which: credit to government (net) $2 / 3 /$ & 15.3 & 6.0 & 1.8 & -98.6 & -96.6 & -3.8 & -1.5 & -1.3 & -0.2 \\
\hline credit to the economy $2 /$ & 20.6 & 34.4 & 11.3 & 24.3 & 13.1 & 12.0 & 10.6 & 10.2 & 10.2 \\
\hline Broad money & 41.8 & 7.4 & 12.3 & 26.1 & 16.1 & 15.4 & 13.2 & 14.3 & 14.4 \\
\hline Velocity (ratio of non-oil GDP to average broad money) & 2.3 & 2.2 & 2.4 & 2.2 & 2.2 & 2.3 & 2.3 & 2.3 & 2.3 \\
\hline Central bank reference interest rate (in percent; end of period) & 14.5 & 14.5 & $\ldots$ & 18.2 & $\ldots$ & $\ldots$ & $\ldots$ & $\ldots$ & $\ldots$ \\
\hline Commercial bank lending rate (in percent; end of period) & 30.0 & 30.0 & $\ldots$ & 32.5 & $\ldots$ & $\ldots$ & $\ldots$ & $\ldots$ & $\ldots$ \\
\hline Commercial bank deposit rate (in percent; end of period) & 10.3 & 10.3 & $\ldots$ & 12.8 & $\ldots$ & $\ldots$ & $\ldots$ & $\ldots$ & $\ldots$ \\
\hline & \multicolumn{9}{|c|}{ (In percent of GDP, unless otherwise specified) } \\
\hline \multicolumn{10}{|l|}{ National accounts } \\
\hline Consumption & 119.0 & 122.1 & 122.0 & 121.8 & 117.2 & 110.4 & 109.8 & 105.3 & 102.9 \\
\hline Gross investment & 36.1 & 35.2 & 37.3 & 36.0 & 42.2 & 51.4 & 55.1 & 55.7 & 53.8 \\
\hline Public investment & 20.1 & 20.1 & 16.4 & 15.2 & 17.5 & 18.4 & 17.5 & 16.4 & 15.3 \\
\hline Private investment & 16.0 & 15.1 & 21.0 & 20.8 & 24.7 & 32.9 & 37.6 & 39.4 & 38.5 \\
\hline Of which: non-oil sector (in percent of non-oil GDP) & 16.1 & 15.1 & 15.1 & 15.1 & 15.1 & 17.5 & 18.0 & 17.0 & 18.3 \\
\hline Gross domestic savings & 15.4 & 16.7 & 10.6 & 4.3 & 14.2 & 22.2 & 22.0 & 23.6 & 22.6 \\
\hline Public savings $4 /$ & 13.4 & 6.8 & 13.4 & 11.6 & 15.5 & 21.9 & 22.7 & 21.7 & 20.5 \\
\hline \multicolumn{10}{|l|}{ Government finance } \\
\hline Total revenue, grants and oil signature bonus & 58.1 & 60.6 & 127.9 & 129.7 & 139.4 & 66.7 & 65.1 & 61.6 & 57.9 \\
\hline Of which: tax revenue & 20.7 & 23.4 & 24.1 & 24.5 & 26.7 & 25.9 & 26.5 & 26.6 & 26.7 \\
\hline grants & 32.5 & 32.2 & 28.2 & 24.6 & 29.0 & 32.9 & 32.0 & 29.0 & 25.5 \\
\hline oil proceeds (signature bonuses and interest) & 0.0 & 0.0 & 71.0 & 74.9 & 78.2 & 1.2 & 0.9 & 0.7 & 0.5 \\
\hline unidentified revenue measures & 0.0 & 0.0 & & 0.0 & 0.0 & 1.5 & 1.3 & 1.1 & 1.1 \\
\hline Total expenditure and net lending & 75.1 & 87.2 & 76.5 & 72.8 & 79.7 & 77.7 & 72.0 & 67.4 & 63.0 \\
\hline Of which: noninterest current expenditure & 28.4 & 41.7 & 37.8 & 38.0 & 38.3 & 34.8 & 32.9 & 30.9 & 29.0 \\
\hline capital and HIPC expenditures & 41.9 & 42.1 & 29.3 & 30.0 & 36.6 & 38.3 & 36.5 & 34.1 & 31.9 \\
\hline Domestic primary balance (commitment basis; incl. HIPC Initiative spending) 5/ & -11.7 & -20.6 & -17.5 & -14.0 & -15.2 & -11.6 & -9.0 & -7.7 & -6.1 \\
\hline Overall balance (commitment basis; including grants) & -17.0 & -26.6 & 51.4 & 56.9 & 59.6 & -11.0 & -6.9 & -5.8 & -5.1 \\
\hline Overall balance (cash basis; including grants) & -11.9 & -20.1 & 27.7 & 37.5 & 54.0 & -11.0 & -6.9 & -5.8 & -5.1 \\
\hline \multicolumn{10}{|l|}{ External sector } \\
\hline Current account balance (including official transfers) & -22.3 & -20.1 & -28.2 & -33.1 & -28.4 & -29.4 & -31.8 & -30.4 & -29.2 \\
\hline Current account balance (excluding official transfers) & -56.7 & -58.9 & -60.3 & -59.2 & -59.8 & -61.9 & -63.5 & -59.3 & -54.7 \\
\hline Net present value of total external debt $6 / 7 /$ & 463.5 & 500.7 & 465.3 & 499.5 & 315.5 & 301.9 & 288.7 & 282.1 & 270.8 \\
\hline Net present value of total external debt $6 / 8$ / & 279.6 & 258.0 & 619.7 & 278.5 & 180.1 & 163.0 & 158.7 & 159.2 & 161.0 \\
\hline Scheduled external debt service before debt relief 9/ & 50.3 & 52.8 & 56.7 & 56.7 & 54.4 & 50.8 & 43.1 & 40.0 & 35.5 \\
\hline External debt service after HIPC 10/ & 10.5 & 11.6 & $\ldots$ & 14.5 & 19.1 & 12.4 & 11.4 & 10.6 & 9.1 \\
\hline Export earnings (in millions of U.S. dollars) & 6.6 & 3.5 & 5.9 & 3.8 & 4.0 & 4.2 & 4.3 & 4.4 & 4.7 \\
\hline Overall balance (in millions of U.S. dollars) & 1.9 & -5.0 & 19.2 & 26.6 & 21.1 & -14.2 & -11.5 & -9.9 & -7.8 \\
\hline Gross foreign reserves (in months of following year's & & & & & & & & & \\
\hline non-oil imports of goods and nonfactor services) 11/ & 4.8 & 3.5 & 3.4 & 3.7 & 3.5 & 3.5 & 3.6 & 3.6 & 3.6 \\
\hline National Oil Account (in millions of U.S. dollars) 12/ & $\ldots$ & $\ldots$ & 23.1 & 23.4 & 47.6 & 39.5 & 32.4 & 26.6 & 21.8 \\
\hline
\end{tabular}

Sources: São Tomé and Príncipe authorities; and staff estimates and projections.

1/ For 2005 corresponds to end-October.

2/ In percent of broad money at beginning of period.

3/ In 2005 and 2006 includes government deposits in the National Oil Account at the BCSTP arising from actual and prospective oil signature bonuses.

IMF Country Report No. 05/323 excluded those deposits.

4/ Government revenue includes HIPC debt relief.

5/ Excluding oil revenue, grants, interest earned and scheduled interest payments, foreign-financed scholarships, and foreign-financed capital outlays. For 2002-04, it also excludes transfers to the JDA, which were repaid in 2005 with proceeds from the oil signature bonus from Block 1 .

6/ Assumes that the completion point under the enhanced HIPC Initiative is in 2006.

7/ In percent of exports of goods and nonfactor services.

8/ In percent of government revenue including grants and excluding oil signature bonuses.

9/ In percent of current year exports of goods and nonfactor services.

10 / Interim debt relief from multilaterals in 2003-2005.

11/ Excluding the National Oil Account and guarantee deposits placed at the central bank by prospective financial institutions pending operating licenses.

12/ Includes interest income. 
Table 2. São Tomé and Príncipe: Financial Operations of the Central Government, 2003-10

\begin{tabular}{|c|c|c|c|c|c|c|c|c|c|c|c|c|c|c|}
\hline & \multirow[t]{3}{*}{2003} & \multirow[t]{3}{*}{2004} & \multicolumn{4}{|c|}{2005} & \multicolumn{4}{|c|}{2006} & \multirow[t]{3}{*}{2007} & \multirow{3}{*}{$\begin{array}{c}2008 \\
\text { Proj. }\end{array}$} & \multirow[t]{2}{*}{2009} & \multirow[t]{2}{*}{2010} \\
\hline & & & \multicolumn{2}{|l|}{$\mathrm{CR} / 05 / 323$} & \multirow{2}{*}{$\frac{\mathrm{CR} / 05 / 323}{\text { Jan-Dec. }}$} & \multirow{2}{*}{$\frac{\text { Est. }}{\text { Jan-Dec. }}$} & \multicolumn{4}{|c|}{ Prog. } & & & & \\
\hline & & & Jan.-Sep. & Jan.-Sep. & & & Jan.-Mar. & Jan.-Jun. & Jan.-Sep. & Jan-Dec. & & & Proj. & \\
\hline & \multicolumn{14}{|c|}{ (in billions of dobras) } \\
\hline Total revenue and grants & 321.0 & 381.2 & 830.7 & 802.7 & 952.8 & 972.1 & 265.6 & 926.1 & $1,070.3$ & $1,239.5$ & 697.9 & 791.8 & 863.5 & \\
\hline Non-oil revenue & 141.5 & 178.7 & 150.4 & 137.1 & 213.8 & 226.5 & 53.9 & 124.7 & 199.4 & 285.6 & 324.8 & 375.7 & 432.2 & \\
\hline Tax revenue & 114.2 & 147.3 & 121.2 & 119.9 & 179.4 & 183.9 & 46.1 & 106.9 & 165.6 & 237.6 & 271.5 & 322.1 & 373.5 & \\
\hline of which: fuel taxes & 18.7 & 29.8 & & 24.2 & & 42.0 & 14.0 & 28.0 & 42.0 & 56.0 & & $=0$. & & \\
\hline Nontax revenue & 27.4 & 31.3 & 29.2 & 17.1 & 34.4 & 42.6 & 7.8 & 17.9 & 33.8 & 48.1 & 53.3 & 53.7 & 58.6 & 66.2 \\
\hline Grants 1/ & 179.4 & 202.6 & 151.4 & 129.6 & 210.1 & 184.1 & 37.7 & 111.2 & 177.9 & 258.1 & 344.0 & 388.8 & 406.5 & 409.5 \\
\hline $\begin{array}{l}\text { Oil signature bonuses (including interest income) } \\
\text { Unidentified revenue measures }\end{array}$ & & & $\begin{array}{r}528.9 \\
\ldots\end{array}$ & $\begin{array}{r}536.1 \\
\ldots\end{array}$ & $\begin{array}{r}528.9 \\
\ldots\end{array}$ & 561.5 & $\begin{array}{r}173.9 \\
0.0\end{array}$ & $\begin{array}{r}690.1 \\
0.0\end{array}$ & $\begin{array}{r}692.9 \\
0.0\end{array}$ & $\begin{array}{r}695.7 \\
0.0\end{array}$ & $\begin{array}{l}13.1 \\
16.0\end{array}$ & $\begin{array}{l}11.3 \\
16.0\end{array}$ & $\begin{array}{r}9.7 \\
15.2\end{array}$ & $\begin{array}{r}8.2 \\
17.8\end{array}$ \\
\hline Total expenditure and net lending & 415.1 & 548.6 & 403.5 & 382.3 & 570.2 & 545.5 & 127.2 & 340.0 & 519.1 & 709.0 & 813.1 & 875.8 & 945.3 & $1,010.9$ \\
\hline Current expenditure & 183.3 & 292.5 & 224.7 & 223.1 & 318.3 & 320.4 & 72.1 & 190.4 & 287.9 & 383.9 & 411.6 & 432.0 & 467.5 & 499.5 \\
\hline Personnel costs & 55.0 & 75.7 & 73.5 & 71.1 & 103.3 & 103.3 & 28.4 & 56.8 & 89.5 & 122.1 & 136.8 & 153.4 & 168.7 & 183.9 \\
\hline Of which: Financed by HIPC relief & & & 3.3 & & 6.7 & & 0.0 & 0.0 & 17.1 & 30.9 & 25.0 & 25.0 & 25.0 & 250 \\
\hline Goods and services $2 \%$ & 43.9 & 95.8 & 43.1 & 46.3 & 58.3 & 65.5 & 11.8 & 24.9 & 49.3 & 71.3 & 77.4 & 81.6 & 87.3 & 91.7 \\
\hline Interest on external debt due & 26.1 & 30.1 & 25.2 & 25.2 & 34.0 & 33.7 & 9.7 & 19.5 & 30.8 & 41.0 & 44.4 & 29.8 & 30.9 & 31.7 \\
\hline Interest on internal debt due & 0.0 & 0.0 & 1.9 & 1.9 & 2.5 & 1.9 & 0.6 & 1.3 & 1.9 & 2.5 & 2.5 & 2.5 & 2.5 & .5 \\
\hline Transfers & 35.9 & 65.9 & 62.9 & 57.7 & 92.6 & 89.8 & 15.1 & 71.0 & 83.9 & 106.4 & 107.6 & 117.8 & 127.5 & 136.8 \\
\hline Of which: Regular transfers & 13.4 & 39.9 & 39.2 & 33.2 & 56.0 & 56.9 & 15.1 & 32.1 & 45.1 & 67.6 & 65.9 & 71.6 & 76.9 & 81.9 \\
\hline Joint Development Agency & 22.6 & 26.0 & 23.7 & 24.5 & 36.6 & 32.9 & 0.0 & 38.8 & 38.8 & 38.8 & 41.7 & 46.2 & 50.6 & 54.8 \\
\hline $\begin{array}{l}\text { Other } \\
\text { Shenditure }\end{array}$ & 22.4 & $\begin{array}{r}25.0 \\
2349\end{array}$ & 18.3 & 20.9 & 27.7 & 26.2 & 6.4 & 17.0 & 32.5 & 40.6 & 42.9 & 46.8 & 50.5 & 53.0 \\
\hline $\begin{array}{l}\text { Capital expenditure } \\
\text { Financed by the }\end{array}$ & $\begin{array}{r}197.9 \\
380\end{array}$ & $\begin{array}{r}234.9 \\
50.5\end{array}$ & 159.0 & 144.8 & $\begin{aligned} 218.2 \\
289\end{aligned}$ & $\begin{array}{c}193.4 \\
15 .\end{array}$ & 50.1 & 131.2 & 199.4 & 267.3 & 310.3 & 352.0 & $\begin{array}{r}382.8 \\
497\end{array}$ & 409.7 \\
\hline $\begin{array}{l}\text { Financed by the treasury } \\
\text { Financed by external sources }\end{array}$ & $\begin{array}{r}38.0 \\
160.0\end{array}$ & $\begin{array}{r}50.5 \\
184.4\end{array}$ & $\begin{array}{r}17.0 \\
142.0\end{array}$ & $\begin{array}{r}10.5 \\
134.3\end{array}$ & $\begin{array}{r}28.9 \\
189.3\end{array}$ & $\begin{array}{c}15.1 \\
178.3\end{array}$ & $\begin{array}{r}2.4 \\
47.7\end{array}$ & $\begin{array}{r}9.1 \\
122.2\end{array}$ & $\begin{array}{r}16.2 \\
183.3\end{array}$ & $\begin{array}{r}23.0 \\
244.3\end{array}$ & $\begin{array}{r}27.6 \\
282.7\end{array}$ & $\begin{array}{r}32.6 \\
319.5\end{array}$ & $\begin{array}{r}49.7 \\
333.0\end{array}$ & $\begin{array}{r}65.9 \\
343.8\end{array}$ \\
\hline $\begin{array}{l}\text { Financed by external sources } \\
\text { HIPC Initiative-related social expenditure }\end{array}$ & $\begin{array}{r}160.0 \\
33.6\end{array}$ & $\begin{array}{r}184.4 \\
30.1\end{array}$ & $\begin{array}{r}142.0 \\
19.8\end{array}$ & $\begin{array}{r}134.3 \\
14.4\end{array}$ & $\begin{array}{r}189.3 \\
33.7\end{array}$ & $\begin{array}{r}178.3 \\
31.7\end{array}$ & $\begin{array}{r}47.7 \\
5.0\end{array}$ & $\begin{array}{r}122.2 \\
18.4\end{array}$ & $\begin{array}{r}183.3 \\
31.7\end{array}$ & $\begin{array}{r}244.3 \\
57.8\end{array}$ & $\begin{array}{r}282.7 \\
91.1\end{array}$ & $\begin{aligned} 319.5 \\
91.7\end{aligned}$ & $\begin{array}{r}333.0 \\
95.1\end{array}$ & 343.8 \\
\hline Net lending & 0.3 & -9.0 & 0.0 & 0.0 & 0.0 & 0.0 & 0.0 & 0.0 & 0.0 & 0.0 & 0.0 & 0.0 & 0.0 & 101.1 \\
\hline Overall fiscal balance (commitment basis) & -94.1 & -167.3 & 427.2 & 420.4 & 382.7 & 426.6 & 138.4 & 586.1 & 551.2 & 530.5 & -115.2 & -83.9 & -81.8 & -8 \\
\hline Change in arrears (net; reduction -) & 28.3 & 41.0 & -174.8 & -79.8 & -176.0 & -145.9 & 2.3 & -50.6 & -50.6 & -50.6 & 0.0 & 0.0 & 0.0 & 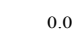 \\
\hline External arrears 3/ & 9.4 & 13.8 & -78.3 & 12.6 & -78.3 & -52.2 & 2.3 & -50.6 & -50.6 & -50.6 & 0.0 & 0.0 & 0.0 & 0 \\
\hline Domestic arrears & 18.8 & 27.2 & -96.5 & -92.4 & -97.7 & -93.6 & 0.0 & 0.0 & 0.0 & 0.0 & 0.0 & 0.0 & 0.0 & 0 \\
\hline Of which: Joint Development Agency & 22.6 & 26.0 & -96.5 & -92.4 & -96.5 & -92.4 & 0.0 & 0.0 & 0.0 & 0.0 & 0.0 & 0.0 & 0.0 & 10 \\
\hline Overall fiscal balance (cash basis) & -65.8 & -126.3 & 252.5 & 340.6 & 206.7 & 280.8 & 140.6 & 535.5 & 500.6 & 479.9 & -115.2 & -83.9 & -81.8 & \\
\hline Financing & 65.8 & 126.3 & -252.5 & -340.6 & -206.7 & -280.8 & -140.6 & -535.5 & -500.6 & -479.9 & 115.2 & 83.9 & 81.8 & is \\
\hline External (net) & 24.7 & 74.2 & -43.2 & -56.5 & -43.8 & -32.1 & -17.9 & -228.4 & -231.0 & -236.7 & -50.9 & -59.5 & -47.3 & -56 \\
\hline Disbursements (projects) & 17.9 & 38.4 & 30.3 & 31.4 & 40.4 & 41.8 & 12.3 & 24.6 & 36.9 & 49.2 & 52.8 & 55.7 & 58.1 & 59.9 \\
\hline Program financing (loans) & 30.8 & 50.3 & 10.4 & 0.0 & 21.6 & 18.0 & 0.0 & 7.8 & 23.1 & 36.5 & 32.3 & 31.9 & 48.1 & 40.1 \\
\hline Short-term loans (net) 4 & 46.7 & 58.7 & -10.4 & 0.0 & -10.4 & 0.0 & 0.0 & -200.4 & -200.4 & -200.4 & 0.0 & 0.0 & $\begin{array}{r}0.0 \\
1535\end{array}$ & 0.0 \\
\hline Scheduled amortization (medium and long-term) & -70.8 & -73.2 & -73.5 & -87.9 & -95.4 & -91.9 & -30.2 & -60.4 & -90.6 & -122.0 & -136.0 & -147.2 & -153.5 & -156.2 \\
\hline Domestic (net) & 16.0 & 17.2 & -27.3 & -41.9 & 5.2 & -16.1 & -53.9 & -40.8 & -16.0 & -6.5 & -15.7 & -7.4 & -7.3 & -1.4 \\
\hline Bank credit (net) & 29.7 & 16.6 & -27.3 & & 5.2 & -16.1 & -53.9 & -40.8 & -16.0 & -6.5 & -15.7 & -7.4 & -7.3 & -1 \\
\hline Nonbank financing & -13.7 & 0.7 & 0.0 & -12.0 & 0.0 & 0.0 & 0.0 & 0.0 & 0.0 & 0.0 & 0.0 & 0.0 & 0.0 & 0.0 \\
\hline Oil reserve fund flows (net) $5 /$ & 0.0 & 0.0 & -245.0 & -280.1 & -245.0 & -276.7 & -80.9 & -307.1 & -309.8 & -312.6 & 116.8 & 101.0 & 86.3 & 73.1 \\
\hline Change in arrears (principal) & 25.1 & 34.9 & -145.6 & 38.0 & -145.6 & -100.0 & 7.3 & -105.4 & -105.4 & -105.4 & 0.0 & 0.0 & 0.0 & 1. \\
\hline $\begin{array}{l}\text { Paris Club rescheduling and debt relief } 6 / \\
\text { Financing gan }\end{array}$ & $\begin{array}{l}0.0 \\
0.0\end{array}$ & 0.0 & $\begin{array}{r}208.6 \\
0.0\end{array}$ & 0.0 & 222.4 & $\begin{array}{r}144.1 \\
00\end{array}$ & $\begin{array}{l}4.8 \\
00\end{array}$ & $\begin{array}{r}146.2 \\
00\end{array}$ & 161.7 & 181.3 & 28.4 & 13.8 & 14.4 & $\begin{array}{c}12.0 \\
530\end{array}$ \\
\hline & & & & & & & & & & & & & & \\
\hline $\begin{array}{l}\text { Memorandum Items: } \\
\text { Domestic primary balance (commitment basis) } 7 /\end{array}$ & -64.9 & -129.3 & -84.4 & -83.9 & -130.6 & -105.1 & -15.2 & -72.4 & -103.7 & -135.6 & -142.6 & -129.9 & -129.3 & \\
\hline Overall balance (commitment basis, excluding Oil Signature Bonuses) & -94.1 & -167.3 & -101.7 & -115.7 & -146.2 & -134.9 & -35.5 & -104.1 & -141.7 & -165.3 & -128.3 & -95.2 & -91.4 & -89.7 \\
\hline Domestic primary spending & 205.3 & 294.3 & 223.9 & 215.1 & 329.4 & 314.6 & 65.1 & 189.1 & 291.1 & 401.2 & 461.9 & 501.8 & 556.0 & 609.4 \\
\hline
\end{tabular}

Sources: São Tomé and Principe authorities; and staff estimates and projection

$\begin{array}{rrrrrr}-94.1 & -167.3 & -101.7 & -115.7 & -146.2 & -134.9 \\ 205.3 & 294.3 & 223.9 & 215.1 & 329.4 & 314.6\end{array}$

2/ In 2004, includes Db20.8 billion (i.e. 3.3 percent of GDP) for hosting the 2004 conference of the Community of Portuguese Language countries (CPLP).

4/ For 2002-04, includes three US\$5 million loans from Nigeria borrowed during 2002-04 and a US\$1 million loan from Angola borrowed in 2004. Repayment of the Nigeria loans has been rescheduled until receipt of oil signature bonuses other than on Block 1. 5/ For 2005 and 2006 net amount is negative on account of the trand in 2006 . Excluding the oil revenue, grants, interest earned and scheduled interest payments, foreign-financed scholarships, and foreign-fire

on Block 1. Includes HIPC Initiative-related social expenditure. 
Table 3. São Tomé and Príncipe: Financial Operations of the Central Government, 2003-10

\begin{tabular}{|c|c|c|c|c|c|c|c|c|c|c|c|c|c|c|}
\hline & \multirow[t]{3}{*}{2003} & \multirow[t]{3}{*}{2004} & \multicolumn{4}{|c|}{2005} & \multicolumn{4}{|c|}{2006} & 2007 & \multirow{2}{*}{\multicolumn{2}{|c|}{2008}} & \multirow[t]{2}{*}{2010} \\
\hline & & & \multirow{2}{*}{\begin{tabular}{|l} 
CR $/ 05 / 233$ \\
Jan.-Se.
\end{tabular}} & & \multirow{2}{*}{$\begin{array}{r}\mathrm{CR} / 05 / 323 \\
\text { Jan-Dec }\end{array}$} & \multirow{2}{*}{$\begin{aligned} \text { Est. } & \text { Jan-Dec }\end{aligned}$} & \multicolumn{4}{|c|}{ Prog. } & \multicolumn{4}{|c|}{ Proj. } \\
\hline & & & & Jan.-Sep. & & & Jan.-Mar. & Jan.Jun. & Jan.-Sep. & $\begin{array}{l}\text { Jan-Dec } \\
\end{array}$ & & & & \\
\hline & \multicolumn{14}{|c|}{ (as a percentage of GDP) } \\
\hline Total revenue and grants & 58.1 & 60.6 & 111.5 & 107.1 & 127.9 & 129.7 & 29.9 & 104.1 & 120.3 & 139.4 & 66.7 & 65.1 & 61.6 & 57.9 \\
\hline Non-oil revenue & 25.6 & 28.4 & 18.7 & 18.3 & 26.7 & 30.2 & 6.1 & 14.0 & 22.4 & 32.1 & 31.0 & 30.9 & 30.8 & 30.8 \\
\hline Tax revenue & 20.7 & 23.4 & 16.3 & 16.0 & 24.1 & 24.5 & 5.2 & 12.0 & 18.6 & 26.7 & 25.9 & 26.5 & 26.6 & 26.7 \\
\hline of which: fuel taxes & 3.4 & 4.7 & & 3.2 & & 5.6 & 1.6 & 3.1 & 4.7 & 6.3 & $\cdots$ & & & $\cdots$ \\
\hline Nontax revenue & 5.0 & 5.0 & 4.0 & 2.3 & 2.6 & 5.7 & 0.9 & 2.0 & 3.8 & 5.4 & 5.1 & 4.4 & 4.2 & 4.1 \\
\hline Grants 1/ & 32.5 & 32.2 & 20.3 & 17.3 & 28.2 & 24.6 & 4.2 & 12.5 & 20.0 & 29.0 & 32.9 & 32.0 & 29.0 & 25.5 \\
\hline $\begin{array}{l}\text { Oil signature bonuses (including interest income) } \\
\text { Unidentified revenue mesarues }\end{array}$ & .... & ... & 71.0 & 71.5 & 71.0 & 74.9 & 19.6 & 77.6 & 77.9 & 78.2 & 1.2 & 0.9 & 0.7 & 0.5 \\
\hline $\begin{array}{l}\text { Unidentified revenue measures } \\
\text { Total expenditure and net lending }\end{array}$ & $\begin{array}{c}\cdots \\
75.1\end{array}$ & 87.2 & 54.2 & $\begin{array}{c}\cdots \\
51.0\end{array}$ & $\begin{array}{c}\cdots \\
76.5\end{array}$ & 72.8 & $\begin{array}{r}0.0 \\
14.3\end{array}$ & 38.2 & $\mathbf{5 8 . 4}$ & $\begin{array}{r}0.0 \\
79.7\end{array}$ & $\begin{array}{r}1.5 \\
77.7\end{array}$ & $\begin{array}{r}1.3 \\
72.0\end{array}$ & $\begin{array}{r}1.1 \\
67.4\end{array}$ & $\begin{array}{r}1.1 \\
63.0\end{array}$ \\
\hline $\begin{array}{l}\text { Current expenditure } \\
\text { S }\end{array}$ & 33.2 & 46.5 & 30.2 & 29.8 & 42.7 & 42.8 & 8.1 & 21.4 & 32.4 & 43.2 & 39.3 & 35.5 & $\begin{array}{l}0.4 \\
33.3\end{array}$ & $\begin{array}{l}03.0 \\
31.1\end{array}$ \\
\hline Personnel costs & 9.9 & 12.0 & 9.9 & 9.5 & 13.9 & 13.8 & 3.2 & 6.4 & 10.1 & 13.7 & 13.1 & 12.6 & 12.0 & 11.5 \\
\hline Of which: Financed by HIPC relief & & & 0.4 & & 0.9 & & 0.0 & 0.0 & 1.9 & 3.5 & 2.4 & 2.1 & 1.8 & 1.6 \\
\hline Goods and services $2 /$ & 7.9 & 15.2 & 5.8 & 6.2 & 7.8 & 8.7 & 1.3 & 2.8 & 5.5 & 8.0 & 7.4 & 6.7 & 6.2 & 5.7 \\
\hline Interest on external debt due & 4.7 & 4.8 & 3.4 & 3.4 & 4.6 & 4.5 & 1.1 & 2.2 & 3.5 & 4.6 & 4.2 & 2.5 & 2.2 & 2.0 \\
\hline Interest on internal debt due & 0.0 & 0.0 & 0.3 & 0.3 & 0.3 & 0.3 & 0.1 & 0.1 & 0.2 & 0.3 & 0.2 & 0.2 & 0.2 & 0.2 \\
\hline Transfers & 6.5 & 10.5 & 8.4 & 7.7 & 12.4 & 12.0 & 1.7 & 8.0 & 9.4 & 12.0 & 10.3 & 9.7 & 9.1 & 8.5 \\
\hline Of which: Regular transfers & 2.4 & 6.3 & 5.3 & 4.4 & 7.5 & 7.6 & 1.7 & 3.6 & 5.1 & 7.6 & 6.3 & 5.9 & 5.5 & 5.1 \\
\hline Joint Development Agency & 4.1 & 4.1 & 3.2 & 3.3 & 4.9 & 4.4 & 0.0 & 4.4 & 4.4 & 4.4 & 4.0 & 3.8 & 3.6 & 3.4 \\
\hline Other & 4.0 & 4.0 & 2.5 & 2.8 & 3.7 & 3.5 & 0.7 & 1.9 & 3.7 & 4.6 & 4.1 & 3.8 & 3.6 & 3.3 \\
\hline Capital expenditure & 35.8 & 37.3 & 21.3 & 19.3 & 29.3 & 25.8 & 5.6 & 14.8 & 22.4 & 30.1 & 29.6 & 28.9 & 27.3 & 25.5 \\
\hline Financed by the treasury & 6.9 & 8.0 & 2.3 & 1.4 & 3.9 & 2.0 & 0.3 & 1.0 & 1.8 & 2.6 & 2.6 & 2.7 & 3.5 & 4.1 \\
\hline Financed by external sources & 28.9 & 29.3 & 19.1 & 17.9 & 25.4 & 23.8 & 5.4 & 13.7 & 20.6 & 27.5 & 27.0 & 26.3 & 23.7 & 21.4 \\
\hline HIPC Initiative-related social expenditure & 6.1 & 4.8 & 2.7 & 1.9 & 4.5 & 4.2 & 0.6 & 2.1 & 3.6 & 6.5 & 8.7 & 7.5 & 6.8 & 6.3 \\
\hline Net lending & 0.1 & -1.4 & 0.0 & 0.0 & 0.0 & 0.0 & 0.0 & 0.0 & 0.0 & 0.0 & 0.0 & 0.0 & 0.0 & 0.0 \\
\hline Overall fiscal balance (commitment basis) & -17.0 & -26.6 & 57.3 & 56.1 & 51.4 & 56.9 & 15.6 & 65.9 & 62.0 & 59.6 & -11.0 & -6.9 & -5.8 & -5.1 \\
\hline Change in arrears (net; reduction -) & 5.1 & 6.5 & -23.5 & -10.6 & -23.6 & -19.5 & 0.3 & -5.7 & -5.7 & -5.7 & 0.0 & 0.0 & 0.0 & 0.0 \\
\hline External arrears 3/ & 1.7 & 2.2 & -10.5 & 1.7 & -10.5 & -7.0 & 0.3 & -5.7 & -5.7 & -5.7 & 0.0 & 0.0 & 0.0 & 0.0 \\
\hline $\begin{array}{l}\text { Domestic arrears } \\
\text {. }\end{array}$ & 3.4 & 4.3 & -13.0 & -12.3 & -13.1 & -12.5 & 0.0 & 0.0 & 0.0 & 0.0 & 0.0 & 0.0 & 0.0 & 0.0 \\
\hline Of which: Joint Development Agency & 4.1 & 4.1 & -13.0 & -12.3 & -13.0 & -12.3 & 0.0 & 0.0 & 0.0 & 0.0 & 0.0 & 0.0 & 0.0 & 0.0 \\
\hline Overall fiscal balance (cash basis) & -11.9 & -20.1 & 33.9 & 45.5 & 27.7 & 37.5 & 15.8 & 60.2 & 56.3 & 54.0 & -11.0 & -6.9 & -5.8 & -5.1 \\
\hline Financing & 11.9 & 20.1 & -33.9 & -45.5 & -27.7 & -37.5 & -15.8 & -60.2 & -56.3 & -54.0 & 11.0 & 6.9 & 5.8 & 5.1 \\
\hline External (net) & 4.5 & 11.8 & -5.8 & -7.5 & -5.9 & -4.3 & -2.0 & -25.7 & -26.0 & -26.6 & -4.9 & -4.9 & -3.4 & -3.5 \\
\hline Disbursements (projects) & 3.2 & 6.1 & 4.1 & 4.2 & 5.4 & 5.6 & 1.4 & 2.8 & 4.1 & 5.5 & 5.0 & 4.6 & 4.1 & 3.7 \\
\hline Program financing (loans) & 5.6 & 8.0 & 1.4 & 0.0 & 2.9 & 2.4 & 0.0 & 0.9 & 2.6 & 4.1 & 3.1 & 2.6 & 3.4 & 2.5 \\
\hline Short-term loans (net) 4/ & 8.5 & 9.3 & -1.4 & 0.0 & -1.4 & 0.0 & 0.0 & -22.5 & -22.5 & -22.5 & 0.0 & 0.0 & 0.0 & 0.0 \\
\hline Scheduled amortization (medium and long-term) & -12.8 & -11.6 & -9.9 & -11.7 & -12.8 & -12.3 & -3.4 & -6.8 & -10.2 & -13.7 & -13.0 & -12.1 & -10.9 & -9.7 \\
\hline Domestic (net) & 2.9 & 2.7 & -3.7 & -5.6 & 0.7 & -2.1 & -6.1 & -4.6 & -1.8 & -0.7 & -1.5 & -0.6 & -0.5 & -0.1 \\
\hline Bank credit (net) & 5.4 & 2.6 & -3.7 & -4.0 & 0.7 & -2.1 & -6.1 & -4.6 & -1.8 & -0.7 & -1.5 & -0.6 & -0.5 & -0.1 \\
\hline Nonbank financing & -2.5 & 0.1 & 0.0 & -1.6 & 0.0 & 0.0 & 0.0 & 0.0 & 0.0 & 0.0 & 0.0 & 0.0 & 0.0 & 0.0 \\
\hline Oil reserve fund flows (net) $5 /$ & 0.0 & 0.0 & -32.9 & -37.4 & -32.9 & -36.9 & -9.1 & -34.5 & -34.8 & -35.2 & 11.2 & 8.3 & 6.2 & 4.6 \\
\hline Change in arrears (principal) & 4.5 & 5.6 & -19.5 & 5.1 & -19.5 & -13.3 & 0.8 & -11.8 & -11.8 & -11.8 & 0.0 & 0.0 & 0.0 & 0.0 \\
\hline $\begin{array}{l}\text { Paris Club rescheduling } 6 / \\
\text { Financing gap }\end{array}$ & $\begin{array}{l}0.0 \\
0.0\end{array}$ & $\begin{array}{l}0.0 \\
0.0\end{array}$ & $\begin{array}{r}28.0 \\
0.0\end{array}$ & $\begin{array}{l}0.0 \\
0.0\end{array}$ & $\begin{array}{r}29.9 \\
0.0\end{array}$ & $\begin{array}{r}19.2 \\
0.0\end{array}$ & $\begin{array}{l}0.5 \\
0.0\end{array}$ & $\begin{array}{r}16.4 \\
0.0\end{array}$ & $\begin{array}{r}18.2 \\
0.0\end{array}$ & $\begin{array}{r}20.4 \\
0.0\end{array}$ & $\begin{array}{l}2.7 \\
3.5\end{array}$ & $\begin{array}{l}1.1 \\
3.0\end{array}$ & $\begin{array}{l}1.0 \\
2.5\end{array}$ & 0.7 \\
\hline \multicolumn{15}{|l|}{ Memorandum items: } \\
\hline Domestic primary balance (commitment basis) $7 /$ & -11.7 & -20.6 & -11.3 & -11.2 & -17.5 & -14.0 & -1.7 & -8.1 & -11.7 & -15.2 & -11.6 & -9.0 & -7.7 & -6.1 \\
\hline Overall balance (commitment basis, excluding Oil Signature Bonuses) & -17.0 & -26.6 & -13.6 & -15.4 & -19.6 & -18.0 & -4.0 & -11.7 & -15.9 & -18.6 & -12.3 & -7.8 & -6.5 & -5.6 \\
\hline Domestic primary spending & 37.1 & 46.8 & 29.9 & 28.7 & 44.2 & 42.0 & 7.3 & 21.3 & 32.7 & 45.1 & 44.1 & 41.3 & 39.6 & 38.0 \\
\hline
\end{tabular}


Table 4. São Tomé and Príncipe: Monetary Survey, 2003-06

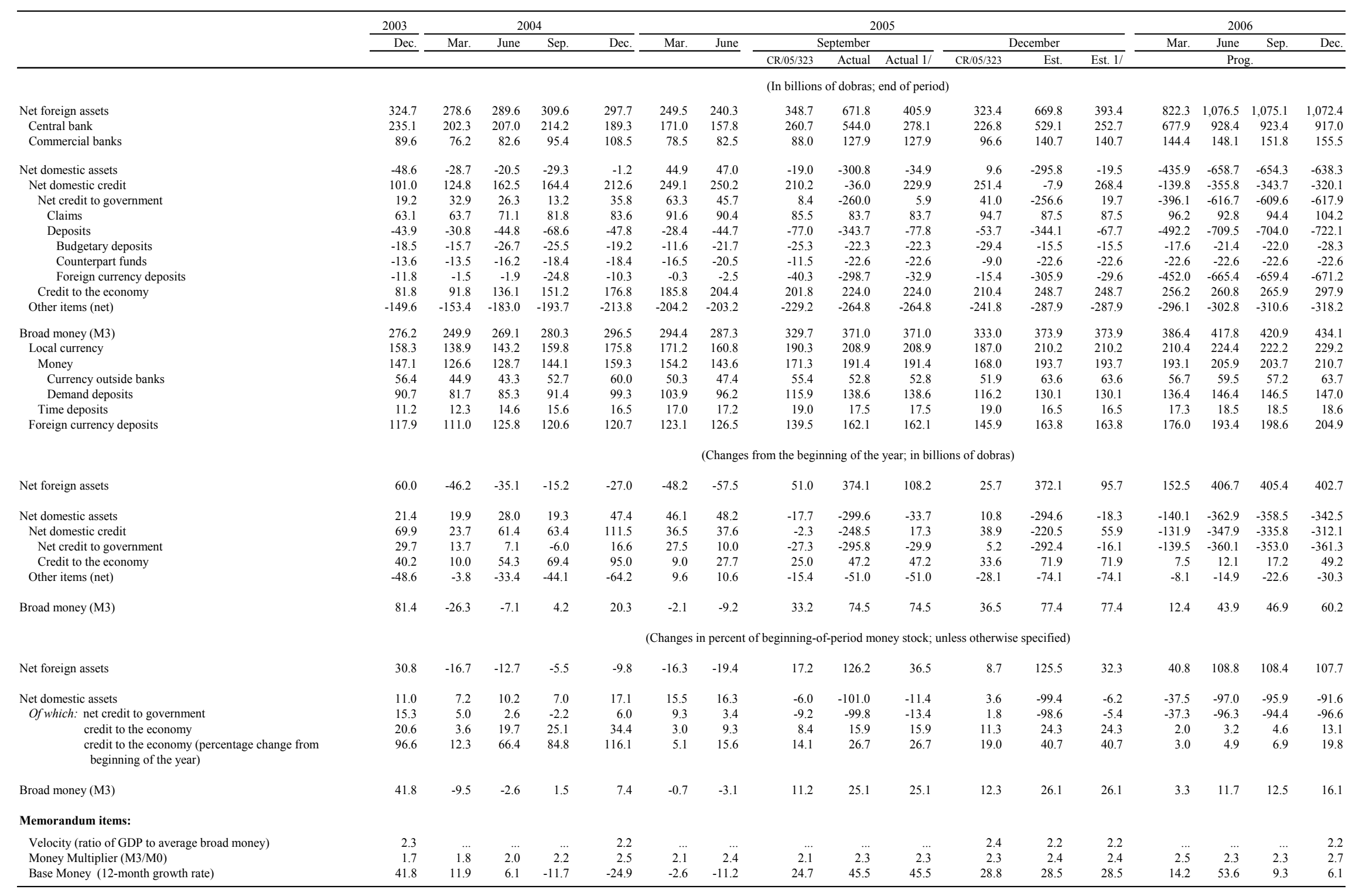

Sources: São Tomé and Príncipe authorities; and staff estimates and projections.

1/ For comparison purposes with the program's monetary numbers (Country Report No. 05/323) balance sheet data for end-September and end-December 2005 have been adjusted by removing the government's National Oil Account

(Conta Nacional de Petroleo, CNP) from the definition of central bank net international reserves and net domestic assets. Originally, the CNP was supposed to be held directly by the government in an account with the Federal Reserve, 
Table 5. São Tomé and Principe: Summary Accounts of the Central Bank, 2003-06

\begin{tabular}{|c|c|c|c|c|c|c|c|c|c|c|c|c|c|c|c|c|c|}
\hline & \multirow{3}{*}{$\frac{2003}{\text { Dec. }}$} & \multicolumn{4}{|c|}{2004} & \multicolumn{8}{|c|}{2005} & \multicolumn{4}{|c|}{2006} \\
\hline & & Mar. & June & Sep. & Dec. & Mar. & June & \multicolumn{3}{|c|}{ September } & \multicolumn{3}{|c|}{ December } & Mar. & & Sep. & Dec. \\
\hline & & & & & & & & CR/05/323 & Actual & Actual $3 /$ & CR/05/323 & Est. & Est. $3 /$ & \multicolumn{3}{|c|}{ Prog. } & \\
\hline & \multicolumn{17}{|c|}{ (In billions of dobras, end of period) } \\
\hline Net foreign assets & 235.1 & 202.3 & 207.0 & 214.2 & 189.3 & 171.0 & 157.8 & 260.7 & 544.0 & 278.1 & 226.8 & 529.1 & 252.7 & 677.9 & 928.4 & 923.4 & 917.0 \\
\hline Gross reserves $1 / 2$ & 240.6 & 232.1 & 206.7 & 221.0 & 197.1 & 190.1 & 175.9 & 277.8 & 549.9 & 284.0 & 252.0 & 526.4 & 250.0 & 682.8 & 929.1 & 924.7 & 927.0 \\
\hline of which: National Oil Account & 0.0 & 0.0 & 0.0 & 0.0 & 0.0 & 0.0 & 0.0 & 0.0 & 265.9 & 0.0 & 0.0 & 276.3 & 0.0 & 361.9 & 595.6 & 613.3 & 631.2 \\
\hline of which: guarantee deposits & 25.6 & 50.5 & 21.1 & 18.7 & 17.9 & 30.0 & 30.0 & 32.9 & 12.7 & 12.7 & 34.2 & 6.5 & 6.5 & 6.7 & 6.8 & 7.0 & 7.2 \\
\hline Short-term liabilities $1 / 2 /$ & -25.6 & -50.5 & -21.1 & -28.6 & -27.9 & -40.0 & -40.0 & -40.1 & -23.4 & -23.4 & -49.0 & -18.3 & -18.3 & -26.4 & -22.8 & -24.0 & -33.3 \\
\hline Other foreign assets & 46.5 & 47.6 & 48.9 & 49.8 & 49.5 & 49.6 & 49.6 & 54.5 & 52.9 & 52.9 & 56.5 & 58.6 & 58.6 & 60.1 & 61.7 & 63.2 & 64.7 \\
\hline Other liabilities & -26.4 & -26.9 & -27.5 & -28.0 & -29.4 & -28.7 & -27.8 & -31.5 & -35.4 & -35.4 & -32.7 & -37.6 & -37.6 & -38.6 & -39.5 & -40.5 & -41.5 \\
\hline Net domestic assets & -76.4 & -61.3 & -72.3 & -88.7 & -70.0 & -33.7 & -38.2 & -107.1 & -361.4 & -95.5 & -82.9 & -375.8 & -99.5 & -521.2 & -744.7 & -741.8 & -754.4 \\
\hline Net domestic credit & 29.4 & 44.0 & 38.7 & 26.6 & 54.7 & 79.8 & 66.1 & 24.9 & -240.2 & 25.7 & 57.5 & -236.5 & 39.8 & -376.8 & -597.5 & -590.4 & -598.7 \\
\hline Net credit to government & 23.8 & 38.3 & 32.1 & 18.8 & 42.6 & 67.6 & 53.5 & 12.7 & -253.0 & 12.9 & 45.3 & -249.6 & 26.8 & -389.0 & -609.6 & -602.6 & -610.9 \\
\hline Claims $2 /{ }^{\circ}$ & 63.1 & 63.7 & 71.1 & 81.8 & 83.6 & 91.6 & 90.4 & 85.5 & 83.7 & 83.7 & 94.7 & 87.5 & 87.5 & 96.2 & 92.8 & 94.4 & 104.2 \\
\hline Of which: use of SDRs/Poverty Reduction and Growth Facility & 35.0 & 35.6 & 36.5 & 37.2 & 38.9 & 38.1 & 36.8 & 48.9 & 44.9 & 44.9 & 58.2 & 48.0 & 48.0 & 56.7 & 66.0 & 67.6 & 77.4 \\
\hline Deposits & -39.2 & -25.4 & -39.0 & -63.0 & -41.0 & -24.0 & -36.9 & -72.7 & -336.7 & -70.8 & -49.4 & -337.0 & -60.7 & -485.2 & -702.4 & -697.0 & -715.1 \\
\hline Ordinary & -16.0 & -12.5 & -23.1 & -22.0 & -14.5 & -7.3 & -13.9 & -21.0 & -15.3 & -15.3 & -25.1 & -8.5 & -8.5 & -10.6 & -14.4 & -15.0 & -21.2 \\
\hline Of which: HIPC Initiative resources & -10.3 & -8.2 & -9.8 & -10.3 & -4.2 & -0.4 & -0.1 & -20.7 & -1.0 & -1.0 & -25.0 & -8.5 & -8.5 & -10.6 & -14.4 & -15.0 & -21.2 \\
\hline Bank liquidation (CNPC; net of claims) & 0.0 & 0.0 & 0.0 & 0.0 & 0.0 & 0.0 & 0.0 & 0.0 & 0.0 & 0.0 & 0.0 & 0.0 & 0.0 & 0.0 & 0.0 & 0.0 & 0.0 \\
\hline $\begin{array}{l}\text { Counterpart funds } \\
\text { (n) }\end{array}$ & -11.5 & -11.4 & -14.1 & -16.2 & -16.2 & -16.5 & -20.5 & -11.5 & -22.6 & -22.6 & -9.0 & -22.6 & -22.6 & -22.6 & -22.6 & -22.6 & -22.6 \\
\hline Foreign currency $2 /$ & -11.8 & -1.5 & -1.9 & -24.8 & -10.3 & -0.3 & -2.5 & -40.3 & -298.7 & -32.9 & -15.4 & -305.9 & -29.6 & -452.0 & -665.4 & $\begin{array}{l}-659.4 \\
-69\end{array}$ & -671.2 \\
\hline of which: National Oil Account & 0.0 & 0.0 & 0.0 & 0.0 & 0.0 & 0.0 & 0.0 & 0.0 & -265.9 & 0.0 & 0.0 & -276.3 & 0.0 & -361.9 & -595.6 & -613.3 & -631.2 \\
\hline of which a amual drawing from oil account & 0.0 & 0.0 & 0.0 & 0.0 & 0.0 & 0.0 & 0.0 & -32.8 & -23.2 & -23.2 & 0.0 & -15.4 & -15.4 & -68.1 & -39.4 & -14.9 & 0.0 \\
\hline Rediscount to commercial banks & 1.1 & 1.1 & 1.2 & 1.2 & 5.2 & 5.2 & 5.2 & 5.2 & 5.2 & 5.2 & 5.2 & 5.2 & 5.2 & 5.2 & 5.2 & 5.2 & 5.2 \\
\hline Other claims & 4.4 & 4.6 & 5.4 & 6.7 & 6.9 & 7.0 & 7.4 & 7.0 & 7.6 & 7.6 & 7.0 & 7.8 & 7.8 & 7.0 & 7.0 & 7.0 & 7.0 \\
\hline Other items (net) & -105.8 & -105.3 & -111.0 & -115.3 & -124.7 & -113.5 & -104.3 & -132.0 & -121.2 & -121.2 & -140.3 & -139.3 & -139.3 & -144.4 & -147.3 & -151.5 & -155.7 \\
\hline Base money & 158.7 & 141.0 & 134.7 & 125.5 & 119.2 & 137.3 & 119.6 & 153.6 & 182.6 & 182.6 & 143.9 & 153.3 & 153.3 & 156.7 & 183.7 & 181.5 & 162.6 \\
\hline Currency issued & 63.1 & 51.6 & 49.4 & 56.6 & 67.2 & 54.2 & 51.3 & 59.3 & 58.1 & 58.1 & 56.9 & 73.2 & 73.2 & 66.3 & 69.1 & 66.8 & 73.3 \\
\hline Bank reserves & 95.6 & 89.4 & 85.3 & 68.9 & 52.1 & 83.0 & 68.3 & 94.3 & 124.5 & 124.5 & 87.1 & 80.1 & 80.1 & 90.4 & 114.5 & 114.7 & 89.3 \\
\hline \multirow{2}{*}{\multicolumn{18}{|c|}{ Memorandum Items: }} \\
\hline & 25.7 & 24.2 & 21.0 & 22.0 & 19.8 & 19.0 & 17.6 & 25.3 & 51.6 & 26.6 & 22.1 & 44.6 & 21.2 & 55.5 & 73.6 & 71.5 & 69.9 \\
\hline of which: excluding National Oil Account and guarantee deposits & 23.0 & 18.9 & 18.8 & 20.1 & 18.0 & 16.0 & 14.6 & 22.3 & 25.4 & ... & 19.1 & 20.6 & ... & 25.5 & 25.9 & 23.5 & 21.8 \\
\hline Net Intermational Reserves (in USS millions) & 23.0 & 18.9 & 18.8 & 19.2 & 17.0 & 15.0 & 13.6 & 21.6 & 49.4 & 24.4 & 17.9 & 43.0 & 19.6 & 53.3 & 71.8 & 69.6 & 67.4 \\
\hline of which: excluding National Oil Account & 23.0 & 18.9 & 18.8 & 19.2 & 17.0 & 15.0 & 13.6 & 21.6 & 24.4 & $\ldots$ & 17.9 & 19.6 & ... & 23.9 & 24.6 & 22.2 & 19.8 \\
\hline National Oil Account (in USS millions) & 0.0 & 0.0 & 0.0 & 0.0 & 0.0 & 0.0 & 0.0 & 23.0 & 24.9 & ... & 23.1 & 23.4 & .... & 29.4 & 47.2 & 47.4 & 47.6 \\
\hline
\end{tabular}

Sources: Såo Tome and Principe authorities; and staff estimates and projection

1/ Includes guarantee deposits by prospective financial institutions pending operating license.

2/ Includes actual and prospective disbursements suder PRGF arrangement.
3/ For comparison purposes with the program's monetary numbers (IMF Country Report No. 05/323) balance sheet data for end-September and end-December 2005 have been adjusted by removing the government's National Oil Account (Conta Nacional de Petroleo, CNP) from the definiti

(l) 
Table 6. São Tomé and Príncipe: Balance of Payments, 2003-10

\begin{tabular}{|c|c|c|c|c|c|c|c|c|c|}
\hline & \multirow[t]{2}{*}{2003} & \multirow[t]{2}{*}{2004} & \multicolumn{2}{|c|}{2005} & \multirow{2}{*}{$\begin{array}{l}2006 \\
\text { Prog. }\end{array}$} & \multirow[t]{2}{*}{2007} & 2008 & 2009 & \multirow[t]{2}{*}{2010} \\
\hline & & & $\mathrm{CR} / 05 / 323$ & Est. & & & $\operatorname{Prc}$ & & \\
\hline & \multicolumn{9}{|c|}{ (In millions of U.S. dollars, unless otherwise specified) } \\
\hline Current account balance (excluding official transfers) & -33.5 & -37.9 & -43.2 & -41.6 & -42.5 & -48.2 & -54.4 & -56.2 & -57.5 \\
\hline Trade balance & -27.0 & -32.5 & -32.1 & -38.3 & -40.0 & -45.1 & -49.7 & -54.1 & -58.8 \\
\hline Exports, f.o.b. 1/ & 6.6 & 3.5 & 5.9 & 3.8 & 4.0 & 4.2 & 4.3 & 4.4 & 4.7 \\
\hline Of which: cocoa & 6.1 & 3.1 & 5.7 & 3.4 & 3.5 & 3.6 & 3.6 & 3.6 & 3.5 \\
\hline Imports, f.o.b. & -33.6 & -36.0 & -38.0 & -42.1 & -44.0 & -49.3 & -54.0 & -58.5 & -63.5 \\
\hline Food & -12.0 & -12.2 & -13.4 & -16.3 & -16.5 & -18.0 & -19.9 & -22.0 & -24.3 \\
\hline Investment goods & -13.4 & -11.6 & -13.6 & -12.2 & -12.9 & -16.4 & -18.6 & -19.9 & -21.3 \\
\hline Petroleum products & -4.7 & -7.8 & -6.2 & -9.9 & -10.8 & -11.1 & -11.4 & -12.1 & -12.8 \\
\hline Other & -3.5 & -4.3 & -4.9 & -3.7 & -3.8 & -3.8 & -4.2 & -4.6 & -5.1 \\
\hline Services and income (net) & -8.4 & -7.5 & -12.6 & -5.3 & -4.6 & -5.2 & -7.2 & -5.1 & -2.3 \\
\hline Exports of nonfactor services & 14.1 & 16.6 & 20.0 & 17.7 & 20.8 & 23.3 & 25.9 & 28.2 & 31.2 \\
\hline Of which : travel and tourism & 10.6 & 12.8 & 13.4 & 13.6 & 15.9 & 17.1 & 18.4 & 19.8 & 21.3 \\
\hline Imports of nonfactor services & -19.7 & -21.0 & -29.7 & -20.0 & -23.0 & -26.2 & -31.8 & -31.9 & -32.0 \\
\hline Freight and insurance & -8.4 & -9.0 & -7.6 & -10.5 & -11.0 & -12.3 & -13.5 & -14.6 & -15.9 \\
\hline Technical assistance & -6.0 & -6.7 & -9.8 & -3.8 & -5.9 & -6.3 & -10.0 & -9.7 & -9.3 \\
\hline Oil sector-related services $2 /$ & 0.0 & 0.0 & -2.4 & 0.0 & 0.0 & 0.0 & 0.0 & 0.0 & 0.0 \\
\hline Other & -5.3 & -5.4 & -9.9 & -5.7 & -6.1 & -7.6 & -8.3 & -7.6 & -6.8 \\
\hline Factor services (net) & -2.8 & -3.1 & -2.8 & -3.0 & -2.4 & -2.3 & -1.3 & -1.4 & -1.5 \\
\hline Interest due & -2.8 & -3.1 & -3.2 & -3.2 & -3.3 & -3.3 & -2.1 & -2.1 & -2.1 \\
\hline Permanent oil fund interest earnings & 0.0 & 0.0 & 0.3 & 0.2 & 0.9 & 1.0 & 0.8 & 0.7 & 0.5 \\
\hline Private transfers (net) & 1.8 & 2.1 & 1.5 & 2.0 & 2.1 & 2.2 & 2.5 & 3.0 & 3.7 \\
\hline Official transfers (net) & 20.3 & 25.0 & 23.6 & 18.4 & 22.3 & 25.3 & 27.2 & 27.4 & 26.8 \\
\hline Of which : Public investment projects & 15.2 & 14.9 & 13.9 & 10.0 & 15.6 & 17.1 & 18.6 & 18.6 & 18.6 \\
\hline Nonproject grants & 0.3 & 2.1 & 1.4 & 2.6 & 0.8 & 0.8 & 1.0 & 1.3 & 0.6 \\
\hline HIPC Initiative-related grants & 3.2 & 3.2 & 5.7 & 3.2 & 3.2 & 4.7 & 5.0 & 5.0 & 5.0 \\
\hline Nigeria grant $3 /$ & 0.1 & 1.4 & 1.4 & 1.4 & 1.6 & 1.6 & 1.4 & 1.4 & 1.4 \\
\hline Current account balance, including official transfers & -13.2 & -13.0 & -19.5 & -23.2 & -20.1 & -22.9 & -27.2 & -28.8 & -30.6 \\
\hline Capital account balance & 15.1 & 8.0 & 38.7 & 49.8 & 41.3 & 8.6 & 15.7 & 19.0 & 22.8 \\
\hline Medium- and long-term capital (net) & 1.0 & 5.1 & 53.5 & 49.0 & 54.4 & -0.2 & 17.2 & 23.7 & 23.7 \\
\hline Project loans & 1.9 & 3.9 & 3.8 & 3.9 & 3.9 & 3.9 & 3.9 & 3.9 & 3.9 \\
\hline Program loans & 3.3 & 5.1 & 2.0 & 1.7 & 2.9 & 2.4 & 2.3 & 3.3 & 2.6 \\
\hline Oil signature bonuses & 0.0 & 0.0 & 49.2 & 49.2 & 54.7 & 0.0 & 0.0 & 0.0 & 0.0 \\
\hline Direct foreign investment & 3.4 & 3.5 & 8.5 & 3.5 & 3.8 & 4.2 & 21.5 & 26.4 & 27.0 \\
\hline Other investment & 0.0 & 0.0 & -1.1 & -0.5 & -1.3 & -0.7 & -0.1 & 0.6 & 0.4 \\
\hline Of which: Use of Fund resources (PRGF) & 0.0 & 0.0 & -1.1 & -0.5 & -1.3 & -0.7 & -0.1 & 0.6 & 0.4 \\
\hline Amortization & -7.6 & -7.5 & -8.9 & -8.9 & -9.6 & -10.1 & -10.4 & -10.4 & -10.2 \\
\hline Short-term capital (incl. errors and ommissions) & 14.0 & 2.9 & -14.7 & 0.8 & -13.1 & 8.9 & -1.5 & -4.8 & -0.9 \\
\hline Of which: $R$ epayment of Nigeria and Angola & 0.0 & 0.0 & -16.0 & 0.0 & -16.0 & 0.0 & 0.0 & 0.0 & 0.0 \\
\hline Transfers to Joint Development Agency & $\cdots$ & $\ldots$ & -12.7 & -12.5 & -3.1 & -3.1 & -3.3 & -3.4 & -3.6 \\
\hline Overall balance & 1.9 & $-\mathbf{5 . 0}$ & 19.2 & 26.6 & 21.1 & -14.2 & -11.5 & -9.9 & -7.8 \\
\hline
\end{tabular}


Table 6. São Tomé and Príncipe: Balance of Payments, 2003-10 (concluded)

\begin{tabular}{|c|c|c|c|c|c|c|c|c|c|}
\hline & \multirow[t]{2}{*}{2003} & \multirow[t]{2}{*}{2004} & \multicolumn{2}{|c|}{2005} & \multirow{2}{*}{$\frac{2006}{\text { Prog. }}$} & 2007 & 2008 & 2009 & \multirow[t]{2}{*}{2010} \\
\hline & & & $\mathrm{CR} / 05 / 323$ & Est. & & \multicolumn{3}{|c|}{ Proj. } & \\
\hline & \multicolumn{9}{|c|}{ (In millions of U.S. dollars, unless otherwise specified) } \\
\hline Overall balance & 1.9 & -5.0 & 19.2 & 26.6 & 21.1 & -14.2 & -11.5 & -9.9 & -7.8 \\
\hline Financing & -1.9 & 5.0 & -19.2 & -26.6 & -21.1 & 14.2 & 11.5 & 9.9 & 7.8 \\
\hline Change in net international reserves (increase -) & -5.9 & 0.0 & -3.0 & -2.7 & -0.5 & -3.0 & -2.0 & -1.5 & -3.5 \\
\hline Use of Fund resources (net) & 0.0 & 0.0 & 1.1 & 0.5 & 1.3 & 0.7 & 0.1 & -0.6 & -0.4 \\
\hline Permanent oil fund (increase -) & 0.0 & 0.0 & -22.8 & -23.3 & -25.0 & 8.7 & 7.1 & 5.8 & 4.8 \\
\hline Change in medium- and long-term arrears (net; decrease -) & 4.0 & 5.0 & -15.3 & -14.6 & -12.5 & 0.0 & 0.0 & 0.0 & 0.0 \\
\hline Debt relief (HIPC bilateral) 5/ & 0.0 & 0.0 & 2.6 & 0.2 & 4.9 & 5.1 & 3.7 & 3.6 & 3.4 \\
\hline Rescheduling arrears & 0.0 & 0.0 & 18.1 & 13.3 & 10.6 & 0.0 & 0.0 & 0.0 & 0.0 \\
\hline Financing gap & 0.0 & 0.0 & 0.0 & 0.0 & 0.0 & 2.7 & 2.6 & 2.4 & 3.6 \\
\hline \multicolumn{10}{|l|}{ Memorandum items: } \\
\hline Cocoa export volume (in thousands of metric tons) & 3.8 & 2.5 & 3.7 & 2.9 & 3.1 & 3.3 & 3.3 & 3.3 & 3.3 \\
\hline Cocoa export unit value (in U.S. dollars per metric ton) & 1,607 & 1,256 & 1,540 & 1,177 & 1,124 & 1,106 & 1,094 & 1,085 & 1,075 \\
\hline Debt-service ratio (before debt relief) $6 / 7 /$ & 50.3 & 52.8 & 56.7 & 56.7 & 54.4 & 50.8 & 43.1 & 40.0 & 35.5 \\
\hline Debt service paid $6 / 8 /$ & 15.4 & 11.8 & $\cdots$ & 109.0 & $\cdots$ & $\cdots$ & $\cdots$ & $\cdots$ & $\cdots$ \\
\hline \multicolumn{10}{|l|}{ Current account balance, including official transfers } \\
\hline \multicolumn{10}{|l|}{ Current account balance, excluding official transfers } \\
\hline (in percent of GDP) & -56.7 & -58.9 & -60.3 & -59.2 & -59.8 & -61.9 & -63.5 & -59.3 & -54.7 \\
\hline \multicolumn{10}{|l|}{ Gross reserves (in months of following year's } \\
\hline non-oil imports of goods and nonfactor services) $9 /$ & 4.8 & 3.5 & 3.4 & 3.7 & 3.5 & 3.5 & 3.6 & 3.6 & 3.6 \\
\hline
\end{tabular}

Sources: São Tomé and Príncipe authorities; and staff estimates and projections.

1/ Nigeria 's aid in kind — previously classified as re-exports — was reclassified as grants.

2/ Covers external payments for services by petrol companies during the course of petroleum exploration.

3/ During 2004-07, includes US\$1.4-1.6 million in aid in kind received from Nigeria.

4/ In 2005 assumes a new Paris Club rescheduling agreement, in 2006 assumes settlement with non-Paris Club creditors.

5/ São Tomé and Príncipe is assumed to reach HIPC completion point in the first half of 2006.

$6 /$ In percent of current year exports of goods and nonfactor services.

7/ Includes obligations to the IMF and arrears.

8/ Includes payments to the IMF and settling of arrears.

9/ Excluding the National Oil Account and guarantee deposits placed at the central bank by prospective financial institutions pending operating licenses.

\section{CInternational Monetary Fund. Not for Redistribution}


Table 7. São Tomé and Príncipe: External Financing Requirements and Sources, 2005-07 (In millions of U.S. dollars)

\begin{tabular}{lrrr}
\hline & 2005 & 2006 & 2007 \\
& Est. & Prog. & Proj. \\
\hline 1. Gross financing requirements & $-\mathbf{6 4 . 1}$ & $\mathbf{- 7 6 . 3}$ & $\mathbf{- 4 9 . 4}$ \\
Current account, excluding current official transfers & -41.6 & -42.5 & -48.2 \\
Exports, f.o.b. & 3.8 & 4.0 & 4.2 \\
Imports, f.o.b. & -42.1 & -44.0 & -49.3 \\
Services and income (net) & -5.3 & -4.6 & -5.2 \\
Private transfers & 2.0 & 2.1 & 2.2 \\
Capital account & -5.2 & -20.8 & 1.8 \\
Scheduled amortization & -8.9 & -9.6 & -10.1 \\
IMF repayments & -0.1 & -0.6 & -0.6 \\
Other capital (net) & 3.9 & -10.6 & 12.4 \\
Change in arrears (net) & -14.6 & -12.5 & 0.0 \\
& & & \\
2. Available Funding & $\mathbf{6 4 . 1}$ & $\mathbf{7 6 . 3}$ & $\mathbf{4 9 . 4}$ \\
Oil Fund (net) & 25.9 & 29.7 & 8.7 \\
Oil signature bonuses & 49.2 & 54.7 & 0.0 \\
Saving (accumulation of oil reserve fund -) & -23.3 & -25.0 & 8.7 \\
Expected disbursements & 24.0 & 29.2 & 31.7 \\
HIPC Initiative-related grants (multilateral creditors) & 3.2 & 3.2 & 4.7 \\
Grants 2/ & 15.2 & 19.2 & 20.7 \\
Concessional loans & 5.6 & 6.8 & 6.3 \\
$\quad$ Project loans & 3.9 & 3.9 & 3.9 \\
$\quad$ Program loans & 1.7 & 2.9 & 2.4 \\
IMF 3/ & 0.6 & 1.8 & 1.2 \\
Financing gap & 13.5 & 15.5 & 7.9 \\
HIPC debt relief (bilateral creditors) 1/3/ & 0.2 & 4.9 & 5.1 \\
Reschedulable arrears (bilateral creditors) 4/ & 13.3 & 10.6 & 0.0 \\
Residual financing gap (actual debt service) & 0.0 & 0.0 & 2.7 \\
\hline & & & \\
\hline
\end{tabular}

Sources: São Tomé and Príncipe authorities; and staff estimates and projections.

1/ Assumes HIPC completion point mid-2006.

2/ Includes US\$1.4 million in aid in kind received from Nigeria.

3/ Includes projected disbursements under new PRGF.

4/ In 2005 includes the new Paris Club rescheduling agreement, in 2006 assumes settlement of arrears with non-Paris Club creditors after conclusion of ongoing reconciliation of debts. 
Table 8. São Tomé and Príncipe: Performance Criteria and Indicative Targets for 2005-06

(In billions of dobras, unless otherwise specified)

\begin{tabular}{|c|c|c|c|c|c|c|c|c|c|c|c|c|}
\hline & \multirow{2}{*}{\multicolumn{4}{|c|}{ end-Sept. }} & \multirow{2}{*}{\multicolumn{4}{|c|}{ end-Dec. }} & \\
\hline & & & & & & & & & \multirow{2}{*}{$\begin{array}{l}\text { end-March } \\
\text { Ind.Target }\end{array}$} & \multirow{2}{*}{$\begin{array}{l}201 \\
\text { end-June } \\
\text { Perf. criteria }\end{array}$} & end-Sept. & end-Dec. \\
\hline & \begin{tabular}{|l} 
Perf. criteria \\
$\mathrm{CR} / 05 / 323$
\end{tabular} & $\begin{array}{l}\text { Perf. Criteria 11// } \\
\text { w/adjusters }\end{array}$ & Actual & $\begin{array}{c}\text { Actual } 12 / \\
\text { excluding } \mathrm{CNP}\end{array}$ & \begin{tabular}{|l} 
Perf. criteria \\
$\mathrm{CR} / 05 / 223$
\end{tabular} & $\begin{array}{c}\text { Perf. Criteria 11/ } \\
\text { w/adjusters }\end{array}$ & Proj. & $\begin{array}{c}\text { Proj. } 12 / \\
\text { excluding CNP }\end{array}$ & & & Ind.Target & Ind.Target \\
\hline 1. Floor on domestic primary balance (as defined in the TMU; cumulative from beginning of year) & -84.4 & -84.4 & -83.9 & -83.9 & -130.6 & -130.6 & -105.1 & -105.1 & -15.2 & -72.4 & -103.7 & -135.6 \\
\hline 2. Ceiling on domestic primary spending (cumulative from beginning of the year) $1 /$ & 223.9 & 223.9 & 215.1 & 215.1 & 329.4 & 329.4 & 314.6 & 314.6 & 65.1 & 189.1 & 291.1 & 401.2 \\
\hline $\begin{array}{l}\text { 3. Ceiling on changes in net domestic financing of the government (cumulative from beginning of year, } \\
\text { in billions of dobras at program exchange rate) } 2 / 3 / 4 /\end{array}$ & -27.6 & -14.6 & -282.8 & -30.9 & 0.8 & -5.3 & -248.4 & -12.1 & -109.8 & -277.9 & -260.5 & -261.9 \\
\hline $\begin{array}{l}\text { 4. Ceiling on changes in net domestic assets of the central bank (cumulative from beginning of year, in } \\
\text { billions of dobras at program exchange rate) } 2 / 3 / 4 /\end{array}$ & -18.5 & -5.5 & -261.7 & -9.8 & 9.9 & 3.8 & -232.7 & 3.7 & -109.8 & -275.9 & -256.5 & -255.9 \\
\hline $\begin{array}{l}\text { 5. Floor on changes in the net international reserves of the central bank (cummulative from beginning of } \\
\text { the year, in millions of U.S. dollars) } 4 / 5 /\end{array}$ & 4.8 & 3.5 & 32.4 & 7.5 & 0.9 & 1.5 & 26.1 & 2.7 & 11.1 & 29.9 & 27.7 & 25.5 \\
\hline $\begin{array}{l}\text { 6. Ceiling on central government's outstanding external payment arrears (stock, in millions of U.S } \\
\text { dollars) } 6 /\end{array}$ & 0.0 & 0.0 & 0.0 & 0.0 & 0.0 & 0.0 & 0.0 & 0.0 & 0.0 & 0.0 & 0.0 & 0.0 \\
\hline $\begin{array}{l}\text { 7. Ceiling on the contracting or guaranteeing of new non-concessional external debt with original maturity } \\
\text { of more than one year by the central government or the BCSTP } \\
\text { (stock, in millions of euros) } 7 / 8 /\end{array}$ & 1.7 & 1.7 & 0.0 & 0.0 & 1.7 & 1.7 & 1.7 & 1.7 & 1.7 & 1.7 & 1.7 & 1.7 \\
\hline $\begin{array}{l}\text { 8. Ceiling on the outstanding stock of external debt with original maturity of less than one year owed or guaranteed } \\
\text { by the central government or the BCSTP (stock, in millions of U.S. dollars) } 9 /\end{array}$ & 15.0 & 15.0 & 1.0 & 1.0 & 15.0 & 15.0 & 1.0 & 1.0 & 1.0 & 1.0 & 1.0 & 1.0 \\
\hline Memorandum items: & & & & & & & & & & & & \\
\hline Base Money (ceiling, in billions of dobras) & $\ldots$ & $\ldots$ & $\ldots$ & $\ldots$ & $\ldots$ & $\ldots$ & $\ldots$ & $\ldots$ & 185.0 & 195.0 & 205.0 & 215.0 \\
\hline Oil signature bonuses (in millions of U.S. dollars, cumulative from beginning of year) & 49.2 & 49.2 & 49.2 & 49.2 & 49.2 & 49.2 & 49.2 & 49.2 & 13.7 & 54.7 & 54.7 & 54.7 \\
\hline Official external program support as defined in the TMU (in billions of dobras at program exchange rate) $10 /$ & 10.4 & 10.4 & 0.0 & 0.0 & 21.6 & 21.6 & 21.6 & 21.6 & 0.0 & 6.1 & 18.2 & 28.3 \\
\hline
\end{tabular}

1/ Equal to government domestic expenditure, as defined in the TMU, excluding all interest payments

2/ The ceiling will be adjusted downward (upward) by the cumulative negative (positive) deviations in external debt serviceand the cumulative positive (negative) deviation of actual from projected disbursements of external program support.

The downward adjustment of higher than programmed external program support will not take place to the extent that the additional support is used to accommodate higher capital outlays of the government, up to US\$1 million

For 2005, actual for end-September and projected for end-December excludes the National Oil Account, consistent with the assumption under IMF Country Report No. 05/323 that such resources would be deposited in a correpondent bank and would not be reflected in Central Bank account

作

$5 /$ The floor on net international reserves will be adjusted upwards (downwards) by the cumulative negative (positive) deviation in external debt service and the cumulative negative (positive) deviation of actual from projected disbursements of external program suppori

The upward adjustment of higher than programmed external program support will not take place to the extent that the additional support is used to accommodate higher capital outlays of the govermment, up to USS1 million.

6/ This is a continuous performance criterion.

7/ This performance criterion applies not only to debt as defined in point No. 9 of the Guidelines on Performance Criteria with Respect to Foreign Debt

adopted on August 24,2000 , but also to commitments contracted or guaranted for which value has not been received.

9. Debt is defined as in point 9 of the Guideling

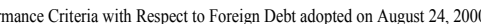

$10 /$ In fiscal tables, valued at projected nominal exchange rates.

$1 /$ sing adusters for shorffalls in program support and foreion debt service.

12/ For comparison purposes with the program's monetary numbers (IMF Country Report No. 05/223, Tables 4 and 5 , and Appendix IV, Table I.1) balance sheet data for end-September and end-December 2005 have been adjusted in Tables 4,5,8 and Appendix I, Table I.1, attached,

by removing the balances in the government's National Oil Account (Conta Nacional de Petroleo, CNP) from the definition of central bank net international reserves and net domestic assets. Originally, the CNP was supposed to be held directly by the govermment in an account with the Federal Reserve 
Table 9. São Tomé and Príncipe: Structural Performance Criteria and Benchmarks for First PRGF Review

Structural Performance Criteria

Status

By end-July 2005, submit to the National

Assembly:

- A new tax code on personal income-taxation,

Done

broadening the tax base and updating the tax rate schedules to provide progressivity to the personal income-tax system;

- A new code on corporate taxation that limits the granting of tax holidays to new licensed investors to a maximum of three years, with tax reductions only covering up to 75 percent of profit taxes due;

- A new tax procedural code establishing tax tribunals at different levels of government to handle tax issues and disputes, as well as penalties and fines for underpayment, late payment and other tax violations; and

- A new investment code that provides for equal treatment of domestic and foreign investors and is consistent with the new code on corporate taxation.

\section{Structural Benchmarks}

- By end-July 2005, establish a timetable for Done the reduction of outstanding payments arrears to the water and electricity company (EMAE).

- By end-July 2005, submit to the National Done Assembly new guidelines on urban property taxation to support the development of new official registries of the quantity, value, and ownership of urban real estate used in apportioning taxes.

- By end-July 2005, submit to the National Done Assembly new legislation on inheritance taxes that clarifies the tax rate and tax base applicable.

- By end-September 2005, submit to the National Assembly proposed legislation criminalizing money laundering and financing of terrorism.
Done

Done 
Table 10. São Tomé and Príncipe: Structural Reform Program at a Glance

\begin{tabular}{|c|c|c|}
\hline Policy Area & Implementation Status & $\begin{array}{c}\text { Funding \& Technical } \\
\text { Assistance }\end{array}$ \\
\hline \multicolumn{3}{|l|}{ Oil sector } \\
\hline $\begin{array}{l}\text { - Issue Oil Revenue Management } \\
\text { Law (ORML) }\end{array}$ & $\begin{array}{l}\text { ORML was approved by the President } \\
\text { of the Republic in December } 2004\end{array}$ & $\begin{array}{l}\text { UNDP, Earth Institute at } \\
\text { Columbia University, and the } \\
\text { World Bank (WB) }\end{array}$ \\
\hline $\begin{array}{l}\text { - Develop overall strategy for } \\
\text { petroleum sector development }\end{array}$ & $\begin{array}{l}\text { A draft strategy, completed in mid- } \\
2005 \text {, is ready for submission to } \\
\text { National Assembly }\end{array}$ & WB \\
\hline $\begin{array}{l}\text { - Develop advisory capacity of the } \\
\text { National Petroleum Agency }\end{array}$ & $\begin{array}{l}\text { A technical assistance program, } \\
\text { including overseas training of local } \\
\text { staff, is underway }\end{array}$ & WB \\
\hline $\begin{array}{l}\text { - Secure transparency and publication } \\
\text { of all documents related to the oil } \\
\text { activity, along the principles of the }\end{array}$ & $\begin{array}{l}\text { The ANP launched its internet website } \\
\text { in December } 2005 \text {. Adoption of EITI } \\
\text { principles being decided upon at the }\end{array}$ & WB \\
\hline Abuja Declaration and EITI & $\begin{array}{l}\text { Joint Ministerial Council (with } \\
\text { Nigeria) level }\end{array}$ & \\
\hline $\begin{array}{l}\text { - Strengthen public dissemination } \\
\text { campaign on oil issues }\end{array}$ & $\begin{array}{l}\text { National forums with the civil society } \\
\text { were held in February, April and in the } \\
\text { Fall of } 2005\end{array}$ & $\begin{array}{l}\text { WB, International Alert, and } \\
\text { Norwegian Government }\end{array}$ \\
\hline \multicolumn{3}{|l|}{$\begin{array}{l}\text { Public expenditure and external debt } \\
\text { management reform }\end{array}$} \\
\hline $\begin{array}{l}\text { - Implement a fully integrated, } \\
\text { computerized budget and public } \\
\text { expenditure management system }\end{array}$ & $\begin{array}{l}\text { Consultants are reviewing the } \\
\text { Treasury's chart of accounts and } \\
\text { setting computer systems }\end{array}$ & WB \\
\hline $\begin{array}{l}\text { - Implement the Commonwealth } \\
\text { Secretariat debt recording } \\
\text { management system to improve the } \\
\text { government's external debt } \\
\text { management capacity }\end{array}$ & $\begin{array}{l}\text { Two expert consultants have been } \\
\text { hired to set system, upload data, and } \\
\text { train STP users }\end{array}$ & WB \\
\hline \multicolumn{3}{|l|}{ Public enterprise reform } \\
\hline $\begin{array}{l}\text { - Launch strategy to improve the } \\
\text { financial position of the water and } \\
\text { electricity company (EMAE) }\end{array}$ & $\begin{array}{l}\text { A comprehensive reform strategy is } \\
\text { underway, MEFP } \mid 24, a\end{array}$ & Government's own resources \\
\hline $\begin{array}{l}\text { - Conduct feasibility studies on the } \\
\text { restructuring of the airport (ENASA) } \\
\text { and seaport (ENAPORT) authority } \\
\text { Judicial sector reform and business } \\
\text { climate }\end{array}$ & $\begin{array}{l}\text { Call for applicants to develop terms of } \\
\text { reference was posted on the internet in } \\
\text { November } 2005\end{array}$ & WB \\
\hline $\begin{array}{l}\text { - Support institutional reform and } \\
\text { capacity building of the judicial } \\
\text { sector, including the establishment of } \\
\text { tribunals of arbitration of businesses } \\
\text { disputes }\end{array}$ & $\begin{array}{l}\text { Draft laws for the judicial sector and } \\
\text { the establishment of tribunals of } \\
\text { arbitration have been prepared, } \\
\text { MEFP } \mid 24, \mathrm{~d} \text {; revisions are underway }\end{array}$ & WB \\
\hline $\begin{array}{l}\text { - Simplify business registration } \\
\text { procedures }\end{array}$ & Strategy is underway, MEFP $24, \mathrm{~d}$ & WB \\
\hline $\begin{array}{l}\text { - Assess property rights for land } \\
\text { holders }\end{array}$ & Pending & Government's own resources \\
\hline
\end{tabular}


Table 11. São Tomé and Príncipe: Millennium Development Goals

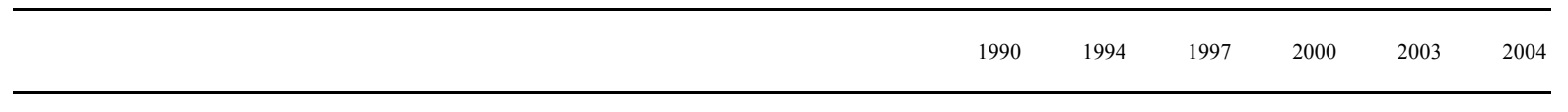

Goal 1. Eradicate extreme poverty and hunger

Target 1: Halve between 1990 and 2015, the proportion of people whose income is less than one dollar a day.

1. Population below US\$ 1 a day (percent)

2 . Poverty gap ratio at US\$ 1 a day (percent)

3. Share of income or consumption held by poorest 20 percent (percent)

Target 2: Halve, between 1990 and 2015, the proportion of people suffering hunger

4. Prevalence of child malnutrition (percent of children under 5)

5. Population below minimum level of dietary energy consumption (percent)

Goal 2. Achieve universal primary education

Target 3: Ensure that, by 2015, children will be able to complete a full course of primary schooling

6. Net primary enrollment ratio (percent of relevant age group)

7. Percentage of cohort reaching grade 5

8. Youth literacy rate (percent age 15-24)

\section{Goal 3. Promote gender equality and empower women}

Target 4: Eliminate gender disparity in primary and secondary education preferably by 2005 and to all levels of education by 2015

9. Ratio of girls to boys in primary and secondary education (percent)

10. Ratio of young literate females to males (percent ages 15-24)

11. Share of women employed in the nonagricultural sector (percent)

12. Proportion of seats held by women in the national parliament (percent)

\section{Goal 4. Reduce child mortality}

Target 5: reduce by two-thirds between 1990 and 2015, the under-five mortality rate

13. Under-five mortality rate (per 1,000)

14. Infant mortality rate (per 1,000 live births)

15. Immunization against measles (percent of children under 12-months)

\begin{tabular}{|c|c|c|c|c|c|}
\hline 118.0 & 118.0 &.. & 118.0 & 118.0 & 118.0 \\
\hline 75.0 & 75.0 &.. & 75.0 & 75.0 & 75.0 \\
\hline & 650 & 600 & 690 & 87.0 & 87.0 \\
\hline
\end{tabular}

Goal 5. Improve maternal health

Target 6: Reduce by three-quarters, between 1990 and 2015, the maternal mortality ratio.

16. Maternal mortality ratio (modeled estimate, per 100,000 live births)

17. Proportion of births attended by skilled health personnel

Goal 6. Combat HIV/AIDS, malaria and other diseases

Target 7: Halt by 2015, and begin to reverse, the spread of HIV/AIDS

18. HIV prevalence among females (percent ages 15-24)

19. Contraceptive prevalence rate (percent of women ages 15-49)

20. Number of children orphaned by HIV/AIDS

Target 8: Halt by 2015, and begin to reverse, the incidence of malaria and other major diseases

21. Prevalence of death associated with malaria

22. Share of population in malaria risk areas using effective prevention and treatment

23. Incidence of tuberculosis (per 100,000 people)

24. Tuberculosis cases detected under DOTS (percent) 
Table 11. São Tomé and Príncipe: Millennium Development Goals (concluded)

\begin{tabular}{|c|c|c|c|c|c|c|}
\hline & 1990 & 1994 & 1997 & 2000 & 2003 & 2004 \\
\hline \multicolumn{7}{|l|}{ Goal 7. Ensure environmental sustainability } \\
\hline \multicolumn{7}{|c|}{$\begin{array}{l}\text { Target 9: Integrate the principles of sustainable development into policies and programs. Reverse the } \\
\text { loss of environmental resources }\end{array}$} \\
\hline 25. Forest area (percent of total land area) & 28.1 &.. & .. & 28.1 & .. & .. \\
\hline 26. Nationally protected areas (percent of total land area) &.. &.. & .. &.. & .. & .. \\
\hline 27. GDP per unit of energy use (PPP \$ per kg oil equivalent) &.. &.. & .. & .. & .. & .. \\
\hline 28. $\mathrm{CO} 2$ emissions (metric tons per capita) & 0.6 & 0.6 & 0.6 & 0.6 & .. & .. \\
\hline 29. Proportion of population using solid fuels & .. &.. & .. &.. & .. & .. \\
\hline \multicolumn{7}{|l|}{ Target 10: Halve by 2015 proportion of people without access to safe drinking water } \\
\hline 30. Access to improved water source (percent of population) &.. & .. & .. &.. & 79.0 & .. \\
\hline \multicolumn{7}{|c|}{ Target 11: Achieve by 2020 significant improvement for at least 100 million slum dwellers } \\
\hline 31. Access to improved sanitation (percent of population) &.. &.. & .. &.. & 24.0 & .. \\
\hline 32. Access to secure tenure (percent of population) &.. &.$\cdot$ & .. &.$\cdot$ & .. & .. \\
\hline \multicolumn{7}{|l|}{ Goal 8. Develop a Global Partnership for Development 2/ } \\
\hline \multicolumn{7}{|l|}{ Target 16: Develop and implement strategies for productive work for youth. } \\
\hline \multicolumn{7}{|l|}{ 45. Unemployment rate of population ages $15-24$ (total) } \\
\hline Female &.. &.. & .. &.. & .. & .. \\
\hline Male & .. & .. & .. &.$\cdot$ & .. & .. \\
\hline \multicolumn{7}{|l|}{ Target 17: Provide access to affordable essential drugs } \\
\hline 46. Proportion of population access with access to affordable essential drugs &. & $\cdot$. &. &.. & .. & .. \\
\hline \multicolumn{7}{|c|}{ Target 18: Make available new technologies, especially information and communications } \\
\hline 47. Fixed line and mobile telephones (per 1,000 people) & 19.2 & 19.8 & 31.2 & 31.0 & 77.6 & 77.6 \\
\hline 48. Personal computers (per 1,000 people) &.. & .. & .. & .. & .. & .. \\
\hline
\end{tabular}

1/ Targets 33-44 are excluded because they can not be measured on a country specific basis. These are related to official development assistance, market access, and the HIPC initiative. 


\section{Table 12: São Tomé and Príncipe-Implementation Status of Floating Completion Point Triggers ${ }^{9}$}

\begin{tabular}{l} 
Trigger Measure \\
\hline Prepare a full PRSP through a participatory process \\
Prepare first annual PRSP progress report \\
Maintain macroeconomic stability, as evidenced by \\
satisfactory PRGF program implementation \\
Assess costing of sectoral strategies for health and \\
education \\
Implement control and monitoring mechanisms to \\
ensure efficient and transparent use of HIPC interim \\
debt relief \\
Place the programming and execution of foreign- \\
financed capital expenditure under the control of the \\
Ministry of Planning and Finance \\
Adopt capacity building program to manage oil \\
resources \\
Establish petroleum revenue oversight and control \\
committee \\
Make operational Auditor General's Office \\
Make operational tribunals of arbitration in business \\
and contract matters \\
Build and equip at least 40 new classrooms in primary \\
schools and 15 classrooms in secondary schools. Also, \\
recruit and train 120 primary school teachers
\end{tabular}

Build and equip at least 8 primary health care centers and increase the immunization rate of children to 85 percent

Reduce the child (under 5) morbidity rate caused by malaria to 60 per 10,000
Implementation Status

Done; Joint Staff Advisory Note (IMF Country Report

No. 05/336) was issued in March 2005.

Expected for early-March 2006.

PRGF-supported program is on track.

Done.

Done.

Done.

Oil Revenue Management Law was approved in December 2004; World Bank's technical support on institution building is ongoing.

Drafting of legislation is ongoing at the National Assembly with World Bank support.

Done

Expected for first quarter of 2006.

As of end-June 2005, the government had built most of the primary and secondary school classrooms agreed to as a HIPC completion trigger point. Between 1999 and 2004, 134 primary school teachers and 174 kindergarten teachers where hired.

7 health centers were built during 1999-2002 and another 2 posts have been re-habilitated. Three of the newly built health centers have been equipped. Vaccination of the children for major childhood diseases (DPT3, Polio, BCG, and measles) has met or surpassed the HIPC completion triggers.

As of 2002 the government had not yet succeeded in reducing the mortality of children (under 5) from malaria; however, the anti-malarial campaign launched in 2003 in Príncipe (education, fumigation, bed nets) and extended in 2004 to the island of São Tomé provides anecdotal evidence of a large drop in infections to date.

\footnotetext{
${ }^{9}$ Floating Completion Point Triggers were reported in the Decision Point Document (of December 5, 2000, page 29 , text box 3 , www.imf.org ).
} 
Table 13. São Tomé and Príncipe: Schedule of Disbursements Under the PRGF Arrangement, 2005-08

\begin{tabular}{llll}
\hline SDR Millions & Percent of Quota & Date available & Disbursement conditions \\
& 5.7 & Aug. 2005 & Board approval of arrangement \\
0.423 & 5.7 & Feb. 2006 & Observance of PCs for end-September 2005 and completion of review \\
0.423 & 5.7 & June 2006 & Observance of PCs for end-December 2005 and completion of review \\
0.423 & 5.7 & Nov. 2006 & Observance of PCs for end-June 2006 and completion of review \\
0.423 & 5.7 & May 2007 & Observance of PCs for end-December 2006 and completion of review \\
0.423 & 5.7 & Nov. 2007 & Observance of PCs for end-June 2007 and completion of review \\
0.423 & 5.7 & May 2008 & Observance of PCs for end-December 2007 and completion of review \\
\hline 0.422 & & \\
\hline
\end{tabular}

Source: Fund Staff. 
Table 14. São Tomé and Príncipe: Indicators of Fund Credit, 2005-10

(In millions of SDRs, unless otherwise indicated)

\begin{tabular}{|c|c|c|c|c|c|c|}
\hline & 2005 & 2006 & 2007 & 2008 & 2009 & 2010 \\
\hline & & \multicolumn{5}{|c|}{ Projections } \\
\hline \multicolumn{7}{|l|}{ Fund credit outstanding 1/ } \\
\hline In millions of SDRs & 2.23 & 3.12 & 3.58 & 3.63 & 3.25 & 2.96 \\
\hline In millions of U.S. dollars & 3.30 & 4.54 & 5.22 & 5.30 & 4.74 & 4.32 \\
\hline In percent of quota & 30.14 & 42.16 & 48.38 & 49.05 & 43.92 & 40.00 \\
\hline \multicolumn{7}{|l|}{ Fund obligations } \\
\hline Fund total charges and interest & 0.03 & 0.03 & 0.03 & 0.03 & 0.03 & 0.03 \\
\hline Existing drawings & 0.03 & 0.03 & 0.02 & 0.02 & 0.02 & 0.02 \\
\hline Prospective drawings & 0.00 & 0.00 & 0.01 & 0.01 & 0.01 & 0.01 \\
\hline Fund total repayment/repurchases & 0.10 & 0.38 & 0.38 & 0.38 & 0.38 & 0.38 \\
\hline Existing drawings & 0.10 & 0.38 & 0.38 & 0.38 & 0.38 & 0.38 \\
\hline Prospective drawings & 0.00 & 0.00 & 0.00 & 0.00 & 0.00 & 0.00 \\
\hline \multicolumn{7}{|l|}{ Fund credit in percent of } \\
\hline Export of goods and nonfactor services & 15.38 & 18.33 & 18.99 & 17.52 & 14.56 & 12.04 \\
\hline Gross official reserves & 16.01 & 20.85 & 21.05 & 19.77 & 16.76 & 13.58 \\
\hline \multicolumn{7}{|l|}{ Memorandum items: } \\
\hline Export of goods and nonfactor services (In millions of US dollars) & 21.47 & 24.78 & 27.47 & 30.23 & 32.56 & 35.86 \\
\hline Gross official reserves (In millions of US dollars) & 20.62 & 21.78 & 24.78 & 26.78 & 28.28 & 31.78 \\
\hline
\end{tabular}

Sources: Fund staff estimates and projections.

1/ Includes the prospective disbursements under the Poverty Reduction and Growth Facility arrangement for a total of SDR2.96 million (40 percent of quota). 
São Tomé, February 9, 2006

Mr. Rodrigo de Rato

Managing Director

International Monetary Fund

Washington, D.C. 20431

Dear Mr. de Rato:

1. This letter and the attached Memorandum of Economic and Financial Policies (MEFP) supplement the letter of intent dated June 30, 2005, and the MEFP of June 2005. The attached MEFP reviews economic developments and policy implementation in 2005. It also sets out the policies that our government intends to implement in 2006 in order to achieve the program objectives. The policy framework for next year is consistent with the macro stabilization and structural reform pursued under the Poverty Reduction and Growth Facility (PRGF) arrangement that was approved by the IMF Executive Board on August 1, 2005. Subject to continued satisfactory performance under the PRGF-supported program and implementation of other applicable conditions, we hope that São Tomé and Príncipe will attain the completion point under the enhanced HIPC Initiative around mid2006.

2. The government of São Tomé and Príncipe has made progress in increasing revenue and controlling the growth of nonprioritary spending to make room for increased povertyrelated spending, strengthening public expenditure management, and using appropriate monetary policy to contain inflation despite a large increase in international oil prices which was fully passed-through to domestic consumers. Looking forward, the policies set out in the attached memorandum, together with a continuing implementation of the government poverty reduction strategy (Estratégia Nacional para Redução da Pobreza; PRSP), aim at solidifying these gains. The performance criteria and benchmarks for end-June 2006 related to the fourth disbursement scheduled for mid-November 2006, as well as the indicative targets for end-March, end-September, and end-December 2006, are set out in Tables I.1 and I.2 of the MEFP. Our intention is to establish end-December 2006 quantitative performance criteria related to the fifth disbursement, scheduled for mid-May 2007 and subject to the fourth review, at the time of the third review scheduled for November 2006, when a new government-following the scheduled parliamentary elections in March 2006-is fully abreast of the economic situation and ready to ratify the policy commitments under the PRGF-supported program.

3. In support of our objectives and policies, the government of São Tomé and Príncipe hereby requests the completion of the First Review and the disbursement of the second tranche under the PRGF in an amount equivalent to SDR 0.423 million, equivalent to 
5.7 percent of quota. In strengthening its overall economic program, the government will continue with a steadfast implementation of the program understandings and resolute efforts to seek additional donor support, as it was done on the occasion of the donor roundtable held in Brussels, under UNDP's auspices, on December 6, 2005.

4. The government of São Tomé and Príncipe will provide the Fund with such information as the Fund may request in connection with the progress made in implementing the economic and financial policies and achieving the objectives of the program.

5. The government of São Tomé and Príncipe believes that the policies and measures set forth in the attached MEFP are adequate to achieve the objectives of the 2006 program supported by the PRGF arrangement, but will take further measures to that end if deemed necessary. During the implementation of the arrangement, the government of São Tomé and Principe will consult with the Managing Director on the adoption of any measures that may be appropriate, at the initiative of the government or whenever the Managing Director requests such a consultation.

6. The government intends to make the contents of this letter and those of the attached MEFP, the technical memorandum of understanding (TMU), and the staff report for this review and the 2005 Article IV consultation, available to the public. In this regard, it authorizes the IMF to arrange for them to be posted on the Fund's website, subsequent to Board completion of the review and conclusion of the 2005 Article IV consultation.

7. We can assure you, Mr. Managing Director, that the government of São Tomé and Príncipe is determined to fully implement the program and we hope we can count on the continued support of the Fund in our endeavors.

Sincerely yours, $/ \mathrm{s} /$

Maria do Carmo Trovoada

Prime Minister and Minister of Planning and Finance

Attachments: Memorandum of Economic and Financial Policies for 2006

Technical Memorandum of Understanding 


\title{
São Tomé and Príncipe: Memorandum of Economic and Financial Policies for 2006
}

\author{
February 2006
}

\section{INTRODUCTION}

1. This Memorandum of Economic and Financial Policies (MEFP) supplements that of June 2005, as well as the letter of intent dated June 30, 2005. It describes (i) performance under the PRGF-supported program up to September 2005, (ii) progress vis-à-vis the December 2005 program targets, and (iii) the government's economic program for 2006 . The thrust of the policies embodied in this MEFP is consistent with the medium-term strategy underpinning the three-year arrangement under the PRGF, which was approved by the IMF's Executive Board on August 1, 2005. Subject to continued satisfactory performance under the PRGF-supported program and implementation of other applicable conditions, we hope that São Tomé and Príncipe will attain the completion point under the enhanced HIPC Initiative around mid-2006.

\section{Performance Under the 2005 Program}

2. During 2005, macroeconomic developments have been broadly satisfactory despite a worsening of the external environment. Leading indicators on the real economy are up: nonoil commodity imports increased by 30 percent between January and August 2005, compared with the same period a year ago, and electricity consumption increased by 13 percent during the same period. Also, the programmed increase in the monthly minimum wage from US\$31 to US\$40 dollars in late-July has kept aggregate demand buoyant. At the same time, however, the large increase in international oil prices registered this year has put pressure on domestic inflation, as these price increases were passed-through to domestic consumers. The (end-period) annual inflation rate for 2005 is currently projected to increase from 15.2 percent in December 2004 to 17 percent in 2005. Our assessment is that at least two percentage points in the current inflation rate are explained by rising international oil prices in the first half of 2005. Electricity tariffs were also increased significantly on November 1, 2005, to address the difficult financial position of the water and electricity company (EMAE). To keep inflation in check and strengthen our program's policy credibility, the Central Bank of São Tomé and Príncipe (BCSTP) increased its reference interest rate from 14.5 percent to 18.2 percent in September 2005. An increased frequency of the foreign exchange auctions during November and December 2005 has also helped to satisfy uphold demand for foreign exchange by relatively large importers, effectively tightening base money at the end of the year. In our view, these monetary policy measures, should lower inflation expectations and deliver a decline in average monthly inflation rates in 2006, consistent with the inflation rate assumed under the program for that year. The tight fiscal stance projected through the end of 2005 should also help arrest aggregate demand pressures in the near-term: the domestic primary deficit is projected at 14 percent of GDP by end-2005, compared with deficit of 17.5 percent of GDP under the PRGF-supported program. 
3. Performance under the PRGF-supported program has been satisfactory. All quantitative performance criteria were observed for end-September 2005. All the structural performance criteria and three structural benchmarks were also met by their scheduled date. This included, notably, the submission to the National Assembly by end-July 2005, of new tax codes on personal and corporate taxation, a new investment code leveling-off the ground for domestic and foreign investors, and new tax administration and tax procedural codes. However, the submission to the National Assembly of legislation criminalizing money laundering and financing of terrorism (structural benchmark for end-September 2005) is still pending while related pieces of legislation are updated. Our plans are to expedite the legislation-updating required during 2006, with a view to submit the legislation to the National Assembly by end-December 2006.

\section{The Macroeconomic Framework for 2006}

4. The macroeconomic outlook is favorable, particularly following the awarding of five additional oil fields located in the zone jointly operated with Nigeria and the rapid progress expected in the completion of the corresponding Production Sharing Agreements (PSAs) with the selected field operators. With respect to Block 4, the expectation is that a new oil consortium would soon be formed following the recent unexpected withdrawal from the group of the selected company to operate the block. Our assessment is that the economy's medium-term growth outlook has improved dramatically. The main challenge for policymakers being the need to secure an orderly transition from the low levels of domestic consumption to those consistent with a fully developed oil sector. Oil revenue in the preproduction period will remain in the form of oil signature bonuses paid by field operators, while the tax regime embodied in the PSAs would determine the government's oil receipts when production starts around 2012/13.

5. Social demands for an increased — and prompt — delivery of basic public services remain unabated. A recent outbreak of cholera in São Tomé highlights the weaknesses of our health services, including the supply of minimum sanitary standards for potable water, as well as shortages with the provision of medical services for those affected by the disease. Social tensions have also been accentuated in recent months among rural population suffering from inadequate roads and infrastructure to access cities. In this context, our view is that these social needs should be a reference benchmark from which to devise and implement economic policies throughout.

6. The 2006 macroeconomic framework is based on gradually accelerating economic growth and declining inflation supported by continued prudent macroeconomic policies and further progress with structural reforms. Real GDP growth is projected to increase from 3.8 percent in 2005 to 4.5 percent in 2006, reflecting fresh initiatives in the infrastructure sector consistent with the implementation of the government's PRSP. This increase is also attributed to private sector investments such as in construction, banking, and trade services, in anticipation of strong growth in the domestic oil sector. Thanks to a prudent monetary policy, inflation is expected to decline from 17 percent in 2005 to 13 percent in 2006. Given the projected private investment, as well as the still-high oil import prices, the external 
current account deficit (including transfers) will remain large, at an equivalent of about 33 percent of GDP. International reserves (excluding the National Oil Account) will remain above three and a half months of imports of goods and services, and will be equivalent to about 150 percent of base money by end-December 2006. Assuming completion point under the Enhanced HIPC initiative by mid-2006, the country's debt service ratios would decline drastically from 56 percent of exports of goods and services in 2005 to 16 percent in 2006 . The implementation of the Multilateral Debt Relief Initiative would further improve São Tomé and Príncipe's debt service ratios after reaching HIPC completion point.

7. Since the debt rescheduling agreement — on Cologne terms — concluded with Paris Club creditors in September 2005, São Tomé and Príncipe has cleared its arrears with the Paris Club creditors. The signature of bilateral agreements with Paris Club creditors is expected to be concluded by April 1, 2006. With respect to its debt to non-Paris Club bilateral creditors, São Tomé and Príncipe will soon start contacting creditors to negotiate debt restructurings in comparable terms to those included in the Paris Club agreement. Our assessment is that the regularization of debts with non-Paris Club countries will only take place in 2006. Under these circumstances, the US\$1.3 million appropriated in the 2005 budget for this debt settlement would remain in an escrow account held by the government at the Central Bank of São Tomé and Príncipe (BCSTP), until payment of these external obligations is made.

\section{A. Fiscal Policy}

8. The fiscal stance during 2006 will be in line with the program supported by the PRGF arrangement, while taking into account recent developments in the oil sector and building upon the various tax measures implemented in 2005. The budget will incorporate part of the second round of oil bonuses concluded in May 2005, with the bulk of the bonus receipts being accumulated in the government's National Oil Account at the BCSTP. The utilization of the oil bonuses will cover projected shortfalls in foreign assistance and the termination of short-term foreign borrowing to finance government spending. Calibration in the use of the bonuses will permit an increase in pro-poor investment outlays consistent with the government's PRSP and will seek to avoid bottlenecks in terms of absorptive capacity. Specifically, the domestic primary deficit will increase from about 14 percent of GDP in 2005 to 15.2 percent in 2006, on account of increased priority spending consistent with the government's PRSP. The composition of total government expenditure will shift in favor of priority spending. Total capital expenditure plus HIPC spending is expected to increase by roughly 7 percentage points of GDP, while current expenditure (excluding budgetary allocations for the holding of parliamentary, regional, and presidential elections) will be decline somewhat as a share of GDP between 2005 and 2006.

9. Total budget revenues (excluding grants and the oil signature bonus receipts) are expected to reach dobras 285.6 billion (i.e., 32.1 percent of GDP, compared with 30.2 percent in 2005), of which dobras 237.6 billion are tax revenues and dobras 48.1 billion are nontax revenues. Attainment of the tax revenue targets for 2006 will be supported by a number of policy initiatives: 
- The full year effect of the tax measures introduced in late 2005--this includes notably the increase in the excise tax levied on services from 2 to 5 percent, and the termination of the deduction on the excise duty charged on domestically-produced beer. Energy taxes will also increase on account of a more depreciated average exchange rate vis-à-vis 2005 , and still high international oil prices.

- Legislation is currently in place to expedite the settlement of tax arrears on corporate and individual income taxes while tackling tax evasion. In particular, for the case of tax arrears accumulated through end-December 2003, settlement with the budget can now be done in up to 18 consecutive monthly tranches rather than requiring a full lump sum payment of the tax liability, as it is currently required by the law. We expect the measure to yield about dobras 1.5 billion through end-June 2006 and dobras 4.0 billion through end-2006 (or 0.45 percent of GDP). To the extent that the end-June target of dobras 1.5 billion fails to materialize, the government will activate, in absence of additional revenue measures, expenditure cuts in consultation with the Fund.

- The reformulation of existing customs procedures with a view to increase the tax base and the effective taxation rate at customs. Currently, many aspects of the Customs Law (Codigo Aduaneiro), and customs procedures and accounting practices date back to the colonial times, thus highlighting the need for reform. Accordingly, it is the government's intention to approve and submit draft proposals to the National Assembly by end-June 2006 (structural benchmark) to revise the Customs Law, the customs' organic law (Estatuto das Alfandigas), and the General Code on Customs Infractions (Regime Geral das Infracós Aduaneiras).

- A strengthening of customs services through better surveillance capacity and an increased number of random inspections of importers' warehouses. The improved customs services will be financed by technical assistance provided by the Millennium Challenge Corporation under its Threshold Program with São Tomé and Príncipe.

10. The effort to control current expenditure is continuing - particularly the wage bill, which will decline marginally as a share of GDP between 2005 and 2006. This objective will be achieved by pursuing a strict management of the payroll through the activation of the civil servants' register that has been recently developed in consultation with the UNDP. Following the sizeable wage increase of July 2005, any further wage increases - consistent with the program's overall wage bill — will only be implemented in the second half of 2006, once a new government is in place. This policy should provide an additional degree of freedom to the new administration, while safeguarding the program's overall spending envelope for 2006. With regard to nonwage expenditure, the government is planning to make savings in several fronts to compensate for new spending connected with the holding of parliamentary, regional, and presidential elections, all of which are scheduled for next year. Despite increases in fuel and electricity prices, expenditure in goods and services will be reduced by 0.7 percentage points of GDP in 2006 on account of expenditure controls across-the-board. 
The projected increase in other expenditure, from 3.5 percent of GDP in 2005 to 4.6 percent in 2006, reflects outlays on three general elections scheduled for 2006, with a total cost to the budget of US $\$ 1$ million (i.e., equivalent to 1.4 percent of GDP or US $\$ 2.2$ dollars per capita cost, per election).

11. The 2006 budget targets an increase in the investment envelope financed by the government's own resources and HIPC (i.e., poverty related) spending. A number of propoor investment projects in agriculture and fisheries have been already prioritized and costed in the context of the PRSP implementation program. Regarding HIPC spending, the challenge from the authorities will be to strike a balance between three competing objectives: (i) a timely compliance with the country's external debt service obligations, particularly following a long period of customary accumulation of debt service arrears, (ii) execution of new poverty-related spending mandated under the HIPC initiative, and (iii) the maintaining of a hard budget constraint for the government (i.e., the level of the domestic primary deficit).

12. As a consequence of this fiscal policy, including the partial utilization of oil signature bonuses and the rescheduling agreement with the Paris Club, net domestic financing of the government in 2006 (excluding the accumulation of deposits in the National Oil Account) will be negative and equivalent to 0.5 percent of GDP. The accumulation of government deposits with the central bank mainly reflects appropriations for poverty-related projects identified under the HIPC initiative, but which are yet to be utilized depending on the outlook for the budget deficit and the preparation of project proposals that could be financed by HIPC resources.

13. To secure compliance with our program's targets for the domestic primary deficit and the domestic primary expenditure, the government will reinforce its expenditure/cash management system underpinning the quarterly projections of government revenue. Specifically, expenditure commitments will be prioritized in line with expected revenue. This approach will allow the Budget Directorate at the Ministry of Planning and Finance to better identify and monitor the seasonality/delays in revenues, grants, and major nontax revenue items (such as fishing licenses, for example), and adapt the expenditure path accordingly.

\section{B. Monetary and Exchange Rate Policy}

14. Monetary policy will aim at reducing inflation to 13 percent by end-2006. Our economic program will keep inflation in check through ceilings on the expansion of the BCSTP's net domestic assets, banking sector credit to the government, and a restrained government expenditure path, against the background of a managed floating exchange rate regime. For end-2006, the stock of net domestic assets of the BCSTP and banking sector credit to the government (excluding the National Oil account) will be negative and limited to dobras 123.1 billion and dobras 13.3 billion, respectively, while the floor on the stock of net international reserves (NIR) of the BCSTP will be set at US\$19.8 million. This floor should allow end-2006 gross international reserves (excluding the National Oil Account and guarantee deposits of banks pending operating licenses) to remain at 3.5 months of imports of goods and services and above 150 percent of base money throughout the year. The 
monetary program assumes a broadly stable velocity of money, which is consistent with broad money growth projected at 16.1 percent. This, in turn will be consistent with a slowdown in private sector credit growth of 19.8 percent in 2006, while safeguarding the banking sector's net external position.

15. During 2006, monetary policy will also aim at buttressing the pre-conditions for successful monetary targeting. Currently, the only central bank instrument to significantly regulate monetary aggregates is the sale of foreign exchange. It is for this reason that our program fast-tracks the development of indirect monetary instruments - in line with technical recommendations from the Fund's Monetary and Financial Systems Department (MFD) which would provide additional instruments to simultaneously target the central bank's NIR and the growth of base money which, in our view, is an important determinant for inflation behavior in the economy. Consistent with this strategy, we have decided to closely monitor base money in our 2006 program.

16. In the meantime, however, the government remains committed to an active monetary policy and will reassess the monetary stance and take actions as needed. The BCSTP's position is that the central bank reference rate should be kept above the inflation rate. Also, depending on the outlook for inflation, the BCSTP will continue to address liquidity concerns through central bank intervention in the foreign exchange markets, as well as appropriate changes in banks' reserve requirements, and/or the issue of liquidity absorbing instruments, such as central bank certificates of deposit (CDs). Coordination of fiscal and monetary policies will also be enhanced in 2006 through regular meetings between the BCSTP and the Ministry of Planning and Finance aimed at forecasting liquidity constraints in the week(s) ahead.

17. The BCSTP will also continue to work closely with MFD regarding the implementation of the technical assistance recommendations in the areas of: (i) banking supervision, (ii) development of interbank money markets, and (iii) foreign exchange auctions. The scope and timing of the foreign exchange auctions will be consistent with the NIR targets set under the program.

18. The BCSTP remains committed to the continuation of the existing managed float exchange rate arrangement, which has allowed the monetary authority to appropriately address the volatility of external inflows and outflows in a context of limited nominal wage flexibility.

\section{External Sector Policies}

19. During 2006, our government will continue to assess major trade policy issues affecting the economy and further strengthen external debt management. However, in assessing São Tomé and Príncipe's participation in bilateral and regional trade arrangements, the government will be mindful of the impact of these memberships on domestic tax revenue collections. 
20. With a view of increasing the tax base and improving customs tax collections, the government has submitted draft legislation to the National Assembly abolishing customs duties exemptions granted to public institutions on imports of raw materials and capital equipment. Tariff exemptions would only remain for grants in kind received by the government. Legislation is also under review by parliament to limit ad hoc customs tax exemptions granted on imports of goods and vehicles by political parties during the preelection campaign. In addition, during 2006, the government will conduct a survey of existing ad hoc customs exemptions with a view to their gradual elimination during the coming years, except for those exemptions contemplated in international agreements. The spirit of this review would be to make legislation consistent with the new investment law currently under consideration by the National Assembly (see paragraph 24, point e, below).

21. The lasting non-tariff barrier related to the privately-managed (though partially stateowned) telecommunications company (CST) will be eliminated by end-December 2005 . Existing regulations protecting CST from competition will expire on that date, opening the sector to new operators. Bidding rounds for different telecommunications services will only start in the second half of 2006, once the sector's regulatory body finalizes all the legislation required for this endeavor as well as the technical analysis and guidelines required for an effective functioning of the telecommunications sector.

22. The government is committed in accepting Article VIII, Sections 2(a), 3, and 4 of the Fund's Articles of Agreement in the near future, and will continue working closely with the Fund's Legal and Monetary and Financial Systems departments to review the relevant laws and regulations. A mission from the IMF visited São Tomé in early-December 2005 to follow-up on these issues.

23. In order to continue improving its external debt management capacity, the Government of São Tomé and Príncipe will install the Commonwealth Secretariat debt recording management system (CS-DRMS) and train the appropriate staff. This will allow the debt unit at the Ministry of Planning and Finance to record all debt transactions and improve debt service programming as well as serve as basis for debt management decision making and analysis. The project is expected to be completed by end-2006 (structural benchmark).

\section{Structural Reforms}

24. The government is committed to building upon current structural reforms, which are critical to enhancing efficiency in fiscal management and stimulating private sector participation in the economy. Some of these measures are also critical to fulfill the floating completion trigger points under the HIPC initiative. In this regard:

a. A comprehensive strategy has been launched to fundamentally improve the financial position of the water and electricity company (EMAE). Key areas of reform include: (i) in line with a resolution (despacho) from the Ministry of Infrastructure, dated October 30, 2005, the phasing-in of an electricity tariff increase, during a six-month period starting November 1, 2005, up to 33 percent 
in the case of individuals and 60 percent in the case of public administration and businesses (e.g., public and private enterprises, international organizations, and parastatal); (ii) the recent establishment of judicial procedures to settle outstanding payments arrears of large electricity customers to EMAE;

(iii) completing the installation of pre-paid electricity metering systems in designated urban locations in April 2006 (structural benchmark); (iv) implementation of a computerized clients' information management system that was developed in December 2005; and (v) the adoption , by end-September 2006, of a sectoral strategy with a view to address, inter alia, the development of suitable infrastructure for electricity production, transportation, and distribution, as well as business modalities to secure dynamic private sector participation in the sector (structural benchmark). In assessing potential public investment for the sector, the government will be mindful of the need to secure concessional financing to avoid exacerbating the already high external indebtedness.

b. A fully integrated, computerized budget and public expenditure management system (SIGFE), developed with assistance from the World Bank, is slated to replace the existing system by end-December 2006 (structural performance criterion). This timing will secure that this accounting system: (i) is tested and operated in parallel with the current reporting system and (ii) is in place for the 2007 budget cycle.

c. The government reaffirms its intention to support the growth of private sector entrepreneurship. In consultation with the World Bank, work is progressing regarding the preparation of terms of reference for the conduct feasibility studies on the public enterprises administering the airports (ENASA) and seaports (ENAPORT). Under the circumstances, however, we will not be able to complete those feasibility studies by end-December 2005 (structural benchmark), as initially programmed. A new deadline for the completion of these studies could be possibly set during the next program's review mission.

d. Necessary actions have been undertaken to improve the business climate in the country. Specifically, by end-January 2006, the government will review the legislation that legalizes businesses only after the company's statutes are published in the national journal (Diario da Republica). At the same time, in an effort to support private entrepreneurship and divulgate best practices to reduce the administrative burdens on start-ups, the government along with the World Bank International Financial Corporation, plans to address ways to modernize the business registration process. This includes the advisability of implementing a one-stop-shop for business registration, reduce informality in the economy, and disseminate information to the general public. Also progressing is the setting of the legal basis to establish tribunals of arbitration to handle business litigation and contract matters. By end-January 2006, the government will submit to parliament a draft law for the judicial sector (Lei Base do Sistema Juridico) that includes regulations on the establishment of these type of tribunals. Also, the government 
has prepared a detailed draft law on tribunals of arbitration (Proposta de Lei de Arbitragem Voluntária) defining the rules of engagement, scope, and responsibilities of those tribunals. It is the government's intention to submit this draft legislation to the National Assembly in February 2006.

e. Approval of critical tax legislation by the National Assembly will take place in two stages during 2006. This legislation was submitted to the National Assembly in the context of the government's 2005 program. The first stage, possible covering through end-March 2006, will include the approval of a new investment code that provides equal treatment to domestic and foreign investors and limits the granting of any tax breaks and exemptions. A new tax code (Codigo General Tributario), a new tax procedural code (Codigo de Procedimiento Tributario), and new guidelines on urban property taxation will also be approved during this first stage of implementation. The second stage will cover the National Assembly's approval of new codes for personal and corporate taxation, and new legislation on inheritance taxes and taxes on transfers of fixed assets (SISA). Significant technical work in consultation with the Fund staff is still underway, given the relevance of these taxes in total tax collections and the potential revenue loss from any drastic reduction in current rates.

\section{E. Transparency and Good Governance}

25. Our government remains committed to strengthening governance and transparency in the management of government resources. The government notes that a formal request has been recently submitted to the Joint Development Agency (JDA) recommending the prompt publication of the PSA on Block 1 signed between the JDA and the selected oil field operator. Also, in support of the rule of law, the government has recently issued an formal declaration ratifying the Supreme Court's ruling that nullified the telecommunication license granted to a second national operator granted in 2004, due to irregularities in the selection process and inconsistencies of this operating permit with the existing provision granted to CST under the existing telecommunications concession agreement (see paragraph 21 above). In addition, the BCSTP has posted in its internet website the external audit of its 2003 and 2004 financial statements, as agreed in the context of the safeguards assessment of the BCSTP conducted by the Fund's Finance department in early 2004. Publication of the audited financial statements will become the norm for the central bank from now on. 
26. For 2006, envisaged policies consistent with the government's PRSP include:

a. The regular updating of the newly-established internet website of the National Petroleum Agency (ANP), with a view to publicize and update all relevant information related to the oil sector on a regular basis.

b. The launching of a thorough reform of the internal oversight/auditing of the financial operations of the central government, public enterprises, and local authorities, performed by the Inspectorate-General of Finance (IGF). As a first step, by end-March 2006, the government will amend the laws defining internal control and administrative regulations governing the IGF's actions (structural benchmark). Also, by end-June 2006, the government will establish a database to record and monitor follow-up actions of problems identified by the IGF in each inspected sector, including the applicable sanctions and penalties (structural benchmark).

c. In cooperation with the World Bank, our government is developing an implementation manual/handbook of the Oil Revenue Management Law of December 2004, which should be divulgated to the population and posted on the internet websites of the National Assembly and the National Petroleum Agency (ANP) by March 2006. The main goal is to identify the administrative bodies that are needed to secure an effective implementation of the letter and spirit of the Law. In addressing this goal, however, the government will remain mindful of the need to strike a balance between a growing bureaucracy and the need to secure transparency and accountability in the management of oil revenue.

d. In cooperation with the Fund's Legal and Monetary and Financial Systems departments, our government will continue reviewing related pieces of legislation to establish a legal framework criminalizing money laundering and the financing of terrorism. Our target is to develop an action plan on the technical steps required for a successful submission of the referred legislation to the National Assembly by end-December 2006. Prospective technical support from USAID to the Prosecutor General's office will also be instrumental in this endeavor.

\section{PRSP AND HIPC INITIATIVE}

27. Our government remains committed to a thorough implementation of São Tomé and Príncipe's PRSP. We intend to submit the first PRSP annual progress report to the Bretton Woods institutions by mid-February 2006. In this regard, the government is working in close collaboration with main donors and civil society to finalize this report as planned.

28. The government is also working in close conjunction with development partners to advance preparations for reaching the HIPC completion point by mid-2006. These tasks 
include the fulfillment of the floating completion triggers and data compilation work required for the preparation of a comprehensive Debt Sustainability Analysis by the staff of the World Bank and the IMF in February 2006.

\section{Program Monitoring FOR 2006}

29. Technical Memorandum of Understanding (TMU). The program will be monitored using the definitions, data sources, and frequency of monitoring set out in the accompanying TMU. The definition of the central bank's net international reserve position (NIR), net domestic assets of the central bank, and banking system net financing to the central government in the TMU reflect the fact that São Tomé and Príncipe's National Oil Account was established with the central bank on behalf of the government, rather than with a foreign custodian bank selected by the government, as it had been initially assumed under the PRGF-supported program. The National Oil Account is properly identified within the central bank accounts, allowing for a transparent management of the oil resources. The government will make available to the Fund all necessary data, appropriately reconciled and on a timely basis, as specified in the TMU.

30. Performance criteria. Table I.1 shows the quantitative performance criteria set for end-June 2006. Structural performance criteria and benchmarks for 2006 with corresponding dates are identified in Table I.2. In addition, the nonaccumulation of external payment arrears (as defined in the TMU) will constitute a continuous performance criterion, as will the standard injunctions against imposing or intensifying restrictions on current payments, introducing or modifying multiple currency practices, concluding bilateral payments agreements that are inconsistent with Article VIII, or imposing or intensifying import restrictions for balance of payments reasons. 
Table I.1. São Tomé and Príncipe: Performance Criteria and Indicative Targets for 2005-06

(In billions of dobras, unless otherwise specified)

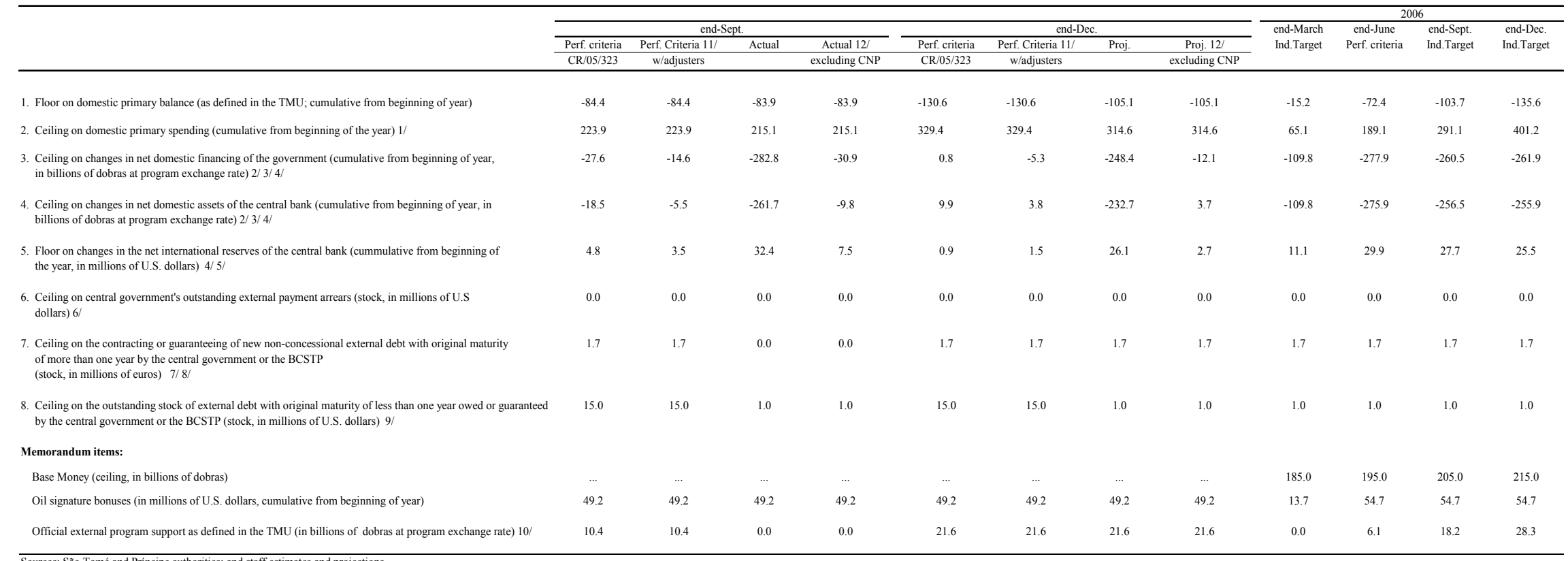

Sources: São Tomé and Príncipe authorities; and staff estimates and projections.

1/ Equal to government domestic expenditure, as defined in the TMU, excluding all interest payments.

The celling will be adjusted downward (upward) by he diwlative negative (posilive) deviations in external debt serviceand the cumulative positive (negative) deviation of actual from projected disbursements of extermal program support.

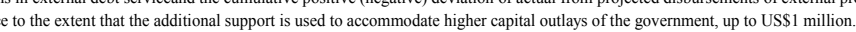

4/For 2005, actual for end-September and projected for end-December excludes the National Oil Account, consistent wit the

tiation in extermal debt service and the cumulative negative (positive) deviation of actual from projected disbursements of external program suppor

The upward adjusment of higher than programmed external program support will not take place to the extent that the additional support is used to accommodate higher capital outlays of the government, up to USS1 million.

(1)

$9 /$ Debt is defined as in point 9 of the Guidelines on Performance Criteria with Respect to Foreign Debb adopted on August 24, 2000.

10/In fiscal tables, valued at projected nominal exchange rates.

12/ For comparison purposes with the program's monetary numbers (IMF Country Report №. 05/323, Tables 4 and 5 , and Appendix IV, Table I.1) balance sheet data for end-September and end-December 2005 have been adjusted in Tables 4, 5, 8 and Appendix I, Table l.1., attached, by removing the

National Oil Account (Conta Nacional de Petroleo, CNP) from the definition of central bank net international reserves and net domestic assets. Originally, the CNP was supposed to be held directly by balances in the government's the government in an account with the Federal Reser 


\section{Table I.2. Structural Performance Criteria and Benchmarks for 2006}

\section{Structural performance criterion}

- By end-December 2006, implement the fully integrated, computerized budget and

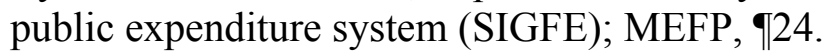

\section{Structural benchmarks}

- By end-March 2006, amend the laws defining internal control and administrative regulations governing the Inspectorate General of Finance (IGF), to increase IGF's powers regarding the oversight and auditing of the financial operations of the central government, public enterprises, and local governments (MEFP, ๆ26).

- By end-June 2006, submit to the National Assembly proposals to revise the Customs Law, the Customs' Organic Law (Estatuto das Alfandigas) and the General Code on Customs Infractions (Regime Geral das Infracós Aduaneiras), to update accounting practices and customs procedures, and modernize the system to increase the efficiency of the overall customs service (MEFP, $\mid 99)$.

- By end-April 2006, complete the installation of EMAE's pre-paid electricity metering systems in designated urban locations with a view to address the public utilities' financial weaknesses in the short-run (MEFP, $\mid 24)$.

- By end-June 2006, establish a database to record and monitor follow-up actions of problems identified by the Inspectorate General of Finance (IGF) in each inspected sector within its jurisprudence, including the applicable sanctions and penalties (MEFP, ๆ26).

- By end-September 2006, adopt a sectoral strategy to address the development of suitable infrastructure for electricity production, transportation, and distribution, as well as business modalities to secure dynamic private sector participation in the electricity sector (MEFP, $\mathbf{q 2 4}$ ).

- By end-December 2006, implement the Commonwealth Secretariat debt recording management system (CS-DRMS) to improve the government's external debt management capacity (MEFP, $₫ 23$ ). 


\section{São Tomé and Príncipe}

\section{Technical Memorandum of Understanding}

1. This technical note contains definitions and adjuster mechanisms that are intended to clarify the measurement of items in Table I.1, Quantitative Performance Criteria, PRGF Arrangement, 2005-06, attached to the Memorandum of Economic and Financial Policies. Unless otherwise specified, all quantitative performance criteria and benchmarks will be evaluated in terms of cumulative flows from December 31, 2005.

\section{Provision of Data to the Fund}

2. Data with respect to all variables subject to performance criteria and indicative benchmarks will be provided to Fund staff on a monthly basis with a lag of no more than four weeks for data on the net domestic assets and net international reserves of the Central Bank of São Tomé and Príncipe (BCSTP), and eight weeks for other data. The authorities will transmit promptly to Fund staff any data revisions. For variables that are relevant for assessing performance against program objectives but are not specifically defined in this memorandum, the authorities will consult with Fund staff as needed on appropriate measurement and reporting.

\section{Definitions}

3. Government is defined for the purposes of this memorandum to comprise the central government. The central government includes all governmental departments, offices, establishments, and other bodies which are agencies or instruments of the central authority of São Tomé and Príncipe. The central government does not include the operations of stateowned enterprises.

4. Government domestic revenue comprises all tax and non-tax revenue of the government (in domestic and foreign currency), excluding foreign grants, the receipts from the local sale of crude oil received from Nigeria as in-kind grant, and any gross inflows to government on account of oil signature bonus payments. Revenue will be measured on a cash basis as reported in the table of government financial operations prepared by the Directorate of Budget and Directorate of Treasury in the Ministry of Planning and Finance.

5. Domestic primary expenditure comprises all government spending assessed on a commitment basis, excluding: (i) foreign-financed capital expenditure, (ii) expenditure under the overseas scholarship program that are externally-financed, and (iii) other foreignfinanced current expenditures, for which data are reported by the Directorate of Budget and Directorate of Treasury. Reporting of government domestic expenditure will be based on the state budget execution prepared every month by the Directorate of Budget and Directorate of Treasury in the Ministry of Planning and Finance. 
6. Within the above total, pro-poor expenditure refers to government outlays that, as it has been agreed with the IMF and World Bank staffs, have a direct effect in reducing poverty. These expenditures, which includes both current and capital outlays, are defined as follows:

a. Pro-poor current spending: These cover the following ministries and expenditure categories (by budget code) as described in the matrix below, with items marked with an " $x$ " representing social expenditures by the referred ministries.

\begin{tabular}{|c|c|c|c|c|}
\hline & & $\begin{array}{l}\text { MINISTRY OF } \\
\text { EDUCATION AND } \\
\text { CULTURE }\end{array}$ & $\begin{array}{l}\text { MINISTRY OF } \\
\text { HEALTH }\end{array}$ & $\begin{array}{c}\text { MINISTRY OF } \\
\text { LABOUR AND } \\
\text { SOLIDARITY }\end{array}$ \\
\hline Code & Description of current expenditure & & & \\
\hline 01.00 .00 & Despesa com Pessoal & $\mathrm{x}$ & $x$ & \\
\hline 02.01 .05 & Outros bens duradouros & $\mathrm{x}$ & $\mathrm{x}$ & \\
\hline 02.02 .02 & Combustíveis e lubrificantes & $\mathrm{x}$ & $\mathrm{x}$ & \\
\hline 02.02 .04 & Alimentação & & $\mathrm{x}$ & \\
\hline 02.02 .05 & Medicamentos & $\mathrm{x}$ & $\mathrm{x}$ & \\
\hline 02.02 .06 & Roupas e calsados & & $\mathrm{X}$ & \\
\hline 02.02 .09 & Outros bens não duradouros & & $x$ & \\
\hline 02.03.01.01 & Agua e energia & $\mathrm{x}$ & $\mathrm{x}$ & \\
\hline 02.03 .02 & Conservação de bens & & $x$ & \\
\hline 02.03.06 & Comunicações & $\mathrm{x}$ & $\mathrm{X}$ & \\
\hline 04.01 .01 & Orçamento do estado & & $x$ & \\
\hline 04.02 .01 & Instituições particulares & & $\mathrm{x}$ & $\mathrm{x}$ \\
\hline 04.03 .01 & Particulares & & $\mathrm{x}$ & $\mathrm{x}$ \\
\hline 04.04.02 & Outras transferências para exterior & & & $\mathrm{x}$ \\
\hline 06.01 .00 & Ensino e formação & $\mathrm{X}$ & & \\
\hline 06.04 .01 & Custos recorrentes de projectos & $x$ & $x$ & \\
\hline 06.04.04.02 & Outros Diversos & $\mathrm{x}$ & $\mathrm{x}$ & \\
\hline
\end{tabular}

Expenditures on "combustiveis e lubrificantes" (fuels and lubricants) that are effected for administrative purposes are excluded. Likewise, "alimentacao" (food) and "roupas e calsados" (clothing and shoes) supplied to administrative staff are excluded.

b. Pro-poor capital spending: This covers selected projects, which are deemed to have a direct impact on alleviating poverty in the following sectors: education, health, social safety nets, agriculture and fisheries, rural development, planning and finance, youth and sports, provision of potable water, and electrification.

c. Reporting Requirements: Data on execution of pro-poor current and capital spending will be provided on a quarterly basis to the staff by the Directorate of Budget (for current expenditure) and by the Directorate of Planning (for capital expenditure) at the Ministry of Planning and Finance. 
7. The domestic primary balance is defined as the difference between government domestic revenue and noninterest government domestic expenditure. This balance for the year 2004 was assessed at dobras -128.1 billion, broken down as follows:

Government domestic revenue:

$\mathrm{Db} 165.0$ billion

Less: government primary expenditure, excluding foreign-financed investment, foreign-financed scholarships, and transfers to the JDA:

Db 293.1 billion

Equals: Domestic primary balance:

$\mathrm{Db}-128.1$ billion

8. The program exchange rate for the purposes of this memorandum will be Db10,104 per US dollar. The exchange rate of the dobra against the euro will be 12,314 and against the SDR will be 15,558 .

9. Net domestic financing of the government is defined as the change in net credit to government by the banking system - that is, the stock of all outstanding claims on the government held by the BCSTP, less all deposits held by the central government with the BCSTP, plus the stock of all outstanding claims on the government held by deposit money banks $(\mathrm{DMBs})^{10}$, less all deposits held by the central government with DMBs--as they are reported monthly by the BCSTP to the IMF. All foreign exchange denominated accounts will be converted to dobras at the program exchange rate. At end-December 2004, outstanding net credit to the government was assessed at dobras 35.8 billion, broken down as follows:

BCSTP credit, including use of IMF resources:

$\mathrm{Db} 83.6$ billion

Less: government deposits with BCSTP:

$\mathrm{Db} 41.0$ billion

Equal: Net credit to government by the BCSTP:

$\mathrm{Db} 42.6$ billion

Plus: DMBs credit:

$\mathrm{Db} \quad 0.0$ billion

Less: government deposits with DMBs (including counterpart funds):

Db 6.9 billion

Equals: Net domestic financing of the government:

Db 35.8 billion

\footnotetext{
${ }^{10}$ Deposit money banks (DMBs) refers to Other Depository Corporations as stated in the Monetary and Financial Statistics Manual.
} 
10. Base money is defined as the sum of currency issued - which consists of currency outside banks and cash in vaults - and bank reserves. Bank reserves refer to reserves of commercial banks held with the central bank, and include reserves in excess of the legal reserve requirement. At end-December 2004, base money was assessed at dobras 119.2 billion, calculated as follows:

Currency issued:

$\mathrm{Db} 67.2$ billion

Of which: $\quad$ Cash in vaults:

$\mathrm{Db} \quad 7.2$ billion

Currency outside banks:

$\mathrm{Db} 60.0$ billion

Plus: Bank Reserves:

$\mathrm{Db} 52.1$ billion

Equals: Base Money:

Db 119.2 billion

11. Net international reserves (NIR) of the BCSTP are defined for program monitoring purposes as short term-term foreign assets of the BCSTP minus short-term external liabilities. All short-term foreign assets that are not fully convertible external assets readily available to and controlled by the BCSTP (i.e., they are pledged or otherwise encumbered external assets, including, but not limited to, the HIPC umbrella SDR account, and assets used as collateral or guarantees for third party liabilities) will be excluded from the definition of NIR. All values are to be converted to U.S. dollars at actual market exchange rates prevailing at the test date. At end-December 2004, NIR was assessed at dobras 169.2 billion, calculated as follows:

Net Foreign Assets:

Db 189.3 billion

Less: Other foreign assets:

$\mathrm{Db} \quad 49.5$ billion

Plus: Other liabilities:

$\mathrm{Db} \quad-29.4$ billion

Equals: Net International Reserves:

$\mathrm{Db} 169.2$ billion

Of which: $\quad$ Gross reserves:

Db 197.1 billion

Short-term liabilities:

$\mathrm{Db}-27.9$ billion

12. Net domestic assets of the central bank of São Tomé and Príncipe are defined as the difference between base money and net foreign assets of the BCSTP. All foreign denominated accounts will be converted to dobras at the program exchange rate. At endDecember 2004, net domestic asset was assessed at dobras -70.0 billion, calculated as follows: 
13. The performance criterion on short-term external debt refers to the outstanding stock of external debt with original maturity of one year or less, including overdraft positions and debt owed or guaranteed by the government or the BCSTP. ${ }^{11}$ At end-December 2004, the stock of short-term external debt stood at US\$1.0 million.

\section{The performance criterion on nonconcessional medium- and long-term external} debt refers to the contracting or guaranteeing of external debt with original maturity of more than one year by the government of BCSTP. ${ }^{12}{ }^{13}$ Debt reschedulings and restructurings are excluded from the ceilings set on nonconcessional borrowing. Medium- and Long-term debt will be reported by the Debt Management Unit of the Ministry of Finance and Planning and (as appropriate) by the BCSTP, measured in US dollars at current exchange rates. The government of São Tomé and Príncipe will consult with Fund staff before contracting obligations if it is uncertain as to whether those obligations are included in the performance criterion limits. At end-December 2004, nonconcessional medium- and long-term external debt stood at US\$15 million.

15. The nonaccumulation of new external payment arrears is a continuous performance criterion. Government external payment arrears are defined as all unpaid external public debt obligations, according to the data established by the Debt Management Unit of the Ministry of Planning and Finance and (as appropriate) by the BCSTP, with the exception of arrears pending rescheduling arrangements. Debt would be deemed to be in arrears when it has not

\footnotetext{
${ }^{11}$ The term "debt" is defined in accordance with point 9 of the Guidelines on Performance Criteria with respect to Foreign Debt (Decision No. 12274-(00/85) August 24, 2000).

${ }^{12}$ This performance criterion applies not only to debt as defined in point No. 9 of the Guidelines on Performance Criteria with Respect to Foreign Debt adopted on August 24, 2000 but also to commitments contracted or guaranteed for which value has not been received.

${ }^{13}$ The concessionality of loans is assessed according to the reference interest rate by currency published by the Development assistance Committee of the Organization for Economic Cooperation and Development (OECD). For loans of terms of no less than 15 years, the ten-year average of commercial interest reference rates (CIRR) for the currency in which the loan is denominated will be used. For loans of shorter terms, the six month average will apply. A loan is deemed to be on concessional terms if, on the initial date of disbursement, the ratio of the present value of the loan, calculated on the basis of the reference interest rate, to the nominal value of the loan is less than 50 percent (in other words, a grant element of at least 50 percent, excluding Fund resources). For currencies with no available reference interest rates, the SDR rate will be used.
} 
been paid by the time it is due, unless the definition of an arrear has been otherwise contractually defined. The performance criterion relating to external arrears does not apply to external arrears pending the conclusion of a debt-rescheduling agreement with the Paris Club. The outstanding stock of external arrears pending debt-rescheduling agreement at endDecember 2004 stood at US\$18 million.

16. Official external program support is defined as grants and loans provided by foreign official entities that are received by the budget - excluding project grants and loans - and other exceptional financing. Amounts assumed in the program consistent with this definition are shown in the memorandum item entitled "external program support" of Table I.1.

\section{Adjusters}

17. Deviations in official external program support from the amounts programmed in Table I.1. will trigger adjusters for domestic financing of the government, net domestic assets of the BCSTP and net international reserves as indicated below. These and other adjusters as set out below will be measured cumulatively from December 31, 2005.

18. Ceilings on net domestic financing (NDF) of the government and net domestic assets (NDA) of the BCSTP. Monthly differences between projected and actual official external program support and external debt service payments in foreign exchange will be converted to dobras at the actual monthly average exchange rate and cumulated to the test date. The ceilings on NDF and NDA will be reduced by the sum of: (i) excess official external program support (as defined in paragraph 16 above); and (ii) the shortfall in external debt service payments. Both ceilings will be increased by 100 percent of any cumulative shortfall in official external program support, or excess in external debt service. The downward adjustment to NDF and NDA for higher than programmed official external program support will not take place to the extent that additional external program support is used to accommodate higher capital outlays by the government, up to US\$1 million, converted to dobras at actual exchange rates, as indicated in Table I.1.

19. Floor on net international reserves (NIR) of the BCSTP. Quarterly difference between projected (see Table I.1) and actual official external program support and external debt service payments will be converted to U.S. dollars at the actual exchange rates prevailing at the test date. The floor on NIR will be raised by the sum of: (i) excess external program support and (ii) any shortfall in external debt service payments. The upward adjustment to NIR for higher than programmed official external program support will not take place to the extent that additional external program support is used to accommodate higher capital outlays by the government, up to US\$1 million, converted to dobras at actual exchange rates, as indicated in Table I.1. The NIR floor will be lowered by 100 percent of any shortfall in official external program support and excess in external debt service payments. 


\section{Data Reporting}

20. The following information will be provided to the IMF staff for the purpose of monitoring the program. Except for net domestic assets and net international reserves, which data are to be provided by the BCSTP within four weeks at the end of each month, other monetary data will be furnished within eight weeks after the end of each month for monthly data, within eight weeks after the end of each quarter for quarterly data, and within eight weeks after the end of each year for annual data.

\section{i. $\quad$ Fiscal data}

The Directorate of Budget at the Ministry of Planning and Finance will provide to IMF staff:

- Monthly data on central government operations for revenues, expenditure and financing

- Monthly detailed tax and non-tax revenues

- Monthly detailed current and capital expenditure data

- Quarterly data on official external program support (non-project)

- Quarterly data on the execution of the public investment program (PIP) and sources of financing

- Quarterly data on project grant disbursement (HIPC and Non-HIPC)

- Quarterly data on project loans disbursement

\section{ii. Monetary data}

The BCSTP will provide the IMF staff:

- Weekly data on exchange rates

- Monthly data on interest rates

- Monthly balance sheet data of BCSTP (in BCSTP and IMF formats)

- Monthly balance sheet data of individual deposit money banks (in BCSTP and IMF formats)

- Monthly consolidated balance sheet data of deposit money banks (in BCSTP and IMF formats)

- Monthly monetary survey (in BCSTP and IMF formats)

- Monthly central bank foreign exchange balance (Balança cambial)

- Latest position of NIR and Liquid Reserves of the BCSTP (last available; monthly) 


\section{iii. External debt data}

The Debt Management Unit at the Ministry of Planning and Finance will provide the IMF staff:

- Monthly data on amortization of external debt (HIPC and Non-HIPC)

- Monthly data on paid interest on external debt (HIPC and Non-HIPC)

- Monthly data on disbursements external project and BOP support loans

\section{iv. National accounts and trade statistics}

The National Institute of Statistics will provide the IMF staff:

- Monthly consumer price index data

- Monthly data on imports as reported by Customs (value and import taxes collected) Monthly commodity export values 


\section{São Tomé And Príncipe: Relations With The FUnd}

(As of December 31, 2005)

I. Membership Status: Joined 09/30/1977; Article XIV

II. General Resources Account:

Quota

Fund holdings of currency

\section{SDR Department:}

Net cumulative allocation

Holdings

IV. Outstanding Purchases and Loans:

Poverty Reduction and Growth Facility

(PRGF) arrangements

V. Financial Arrangements:

$\begin{array}{lcccc}\text { Type } & \begin{array}{c}\text { Approval } \\ \text { date }\end{array} & \begin{array}{c}\text { Expiration } \\ \text { date }\end{array} & \begin{array}{c}\text { Amount approved } \\ \text { (SDR million) }\end{array} & \begin{array}{c}\text { Amount drawn } \\ \text { (SDR million) }\end{array} \\ \text { PRGF } & 08 / 01 / 2005 & 07 / 31 / 2008 & 2.96 & 0.42 \\ \text { PRGF } & 04 / 28 / 2000 & 04 / 27 / 2003 & 6.66 & 1.90 \\ \text { ctural } & & & & 0.80\end{array}$

Percent

$\underline{\text { SDR million }}$

7.40

7.40

$\underline{\text { SDR million }}$

0.62

0.42

$\underline{\text { SDR million }}$

2.33
Percent

Quota

31.42

Quota

100.00

100.05

Percent

Allocation

100.00

68.48
Facility (SAF) 06/02/1989 06/01/1992

2.80

0.80 


\section{Projected Obligations to Fund}

(SDR million; based on existing use of resources and present holdings of SDRs):

\begin{tabular}{lccccc} 
& \multicolumn{5}{c}{ Forthcoming } \\
Principal & $\underline{2005}$ & $\underline{2006}$ & $\underline{2007}$ & $\underline{2008}$ & $\underline{2009}$ \\
Charges/interest & 0.10 & 0.38 & 0.38 & 0.38 & 0.38 \\
Total & 0.01 & 0.02 & 0.01 & 0.01 & 0.01 \\
& 0.10 & 0.40 & 0.39 & 0.39 & 0.39
\end{tabular}

\section{Implementation of HIPC Initiative:}

Enhanced

framework

Commitment of HIPC assistance

Decision point date

$12 / 20 / 00$

Assistance committed (NPV terms) ${ }^{1}$

Total assistance (US\$ million)

97.00

Of which: Fund assistance (SDR million)

Completion point date

Disbursement of IMF assistance (SDR million)

Assistance disbursed to the member

0.00

Interim assistance

0.00

Completion point

0.00

Amount applied against member's obligations

(cumulative)
0.00

\footnotetext{
${ }^{1}$ Net present value (NPV) terms at the decision point under the enhanced framework.
} 


\section{Safeguards Assessments:}

Under the Fund's safeguards assessment policy, the Central Bank of São Tomé and Príncipe is subject to a full safeguards assessment with respect to a forthcoming arrangement. The assessment, which was completed on August 2, 2004, found vulnerabilities in the area of financial reporting, internal audit and internal control and measures to strengthen the control framework and help safeguard Fund resources were proposed. Recommendations are in the process of implementation.

\section{Exchange Arrangements:}

São Tomé and Príncipe's exchange rate regime is currently classified by the Fund as managed floating. The official exchange rate is determined on a daily basis as a weighted average of the previous day's selling rates in the commercial banks, and the outcomes of central bank's foreign exchange auctions. The spread between the official and parallel market exchange rates has been less than 1 percent since 1999. The intervention currency for the dobra is the U.S. dollar. The official exchange rate was Db 11,929.7 per US dollar on December 31, 2005.

\section{Article IV Consultation:}

The last Article IV consultation with São Tomé and Príncipe was concluded by the Executive Board on March 17, 2004.

\section{Financial Sector Assessment Program (FSAP), Reports on Observance of Standards and Codes (ROSCs), and Offshore Financial Center (OFC) Assessments:}

None.

\section{Technical Assistance:}

August/September 2005: MFD mission on banking supervision and foreign exchange operations.

February 2005: STA technical assistance mission on national accounts statistics.

January 2005: MFD mission on monetary operations and liquidity management. 
December 2004: STA mission to advice on compilation and reporting of monetary statistics.

October 2004: STA technical assistance mission on government finance statistics.

September 2004: MFD multi-sector mission to develop foreign exchange and interbank money markets.

July-September 2004: MFD expert mission on Banking Supervision.

June 2004: STA technical assistance mission on balance of payments statistics.

July 2004: MFD expert mission on Monetary Operations.

June 2003: STA technical assistance mission on balance of payments statistics.

March and June 2003: STA technical assistance missions on national accounts statistics.

March 2003 and April 2004: Visits by MFD advisors on the conduct of monetary policy and bank supervision.

March 2003: STA technical assistance mission on national accounts statistics.

January 1998-December 2001: MFD advisor on the conduct of monetary policy, banking supervision, and foreign exchange management.

June 2001: MFD mission on handling of banking crisis and foreign exchange market organization.

\section{Resident Representative:}

A Fund Resident Representative office has been maintained in since November 1999. The current Resident Representative, Mr. Randriamaholy, took up his post in October 2003. 


\section{São Tomé And Príncipe: Relations With The World Bank Group}

\section{IDA operations}

1. São Tomé and Príncipe joined the World Bank and IDA in 1977. New lending was suspended between 1992 and 2000 owing to the lack of an appropriate macroeconomic framework.

2. The World Bank FY06-09 country assistance strategy was well received by the Board in June 2005: its two principal objectives are: (i) to support the implementation of the PRSP; and (ii) to provide timely assistance to ensure that the country is sufficiently prepared to maximize the benefits of oil revenues. Given the small size of our program, the CAS remains tightly focused on on-going activities in the oil sector, social sectors, public sector management and assistance with the HIPC Initiative.

3. Despite its small project portfolio, the Bank's program is relatively large for such a small country as there is a broad ESW and AAA program currently covering oil issues, support to PRSP implementation, assistance with achieving HIPC Completion Point and analysis of trade issues through the multi-sectoral Trade Diagnostic study. The World Bank also supports government reforms by providing advisory services in the petroleum sector, telecommunications and environment. An IDF (Institutional Development Grant) was approved in early 2005 to assist the government in its Judicial reform program. A GEF (Global Environmental Fund) was approved in late 2004 to support the development of a national action plan to adapt to environmental and global warming impacts.

4. Current IDA commitments for the 2 ongoing projects amount to US\$11.5 million with US\$9.9 million undisbursed. The Bank supports the health and the education sectors through its Social Sector Project of US\$6.5 million (of which US\$1.5 million is in the form of an IDA grant) and supports the PRSP, public expenditure management and petroleum sector through its Governance Capacity Building Technical Assistance Project of US\$5 million. The two projects are rated Satisfactory although portfolio performance is hampered by weak management and absorptive capacity.

\section{IFC operations}

5. São Tomé and Príncipe is not a member of the IFC. FIAS has reviewed the country's tax system and private sector investment. 
Statement of IDA Credits as of August 31, 2005

(In millions of U.S. dollars)

\begin{tabular}{|c|c|c|c|c|}
\hline $\begin{array}{l}\text { Credit } \\
\text { Number }\end{array}$ & $\begin{array}{l}\text { Fiscal } \\
\text { Year }\end{array}$ & \multicolumn{3}{|c|}{ IDA } \\
\hline 1590 & 1985 & Economic rehabilitation & 5.0 & 0.0 \\
\hline A029 & 1987 & Special African Facility & 3.0 & 0.0 \\
\hline 1825 & 1987 & Structural adjustment credit & 4.0 & 0.0 \\
\hline 1830 & 1987 & Cocoa rehabilitation credit & 7.9 & 0.0 \\
\hline 2038 & 1989 & Multisector credit & 5.0 & 0.0 \\
\hline 2165 & 1990 & $\begin{array}{l}\text { Second structural adjustment } \\
\text { credit }\end{array}$ & 9.8 & 0.0 \\
\hline 2280 & 1991 & Multisector II credit & 6.0 & 0.0 \\
\hline 2325 & 1991 & Agricultural sector & 9.8 & 0.3 \\
\hline 2343 & 1992 & Health and education & 11.4 & 0.5 \\
\hline 3428 & 2000 & $\begin{array}{l}\text { Public resource management } \\
\text { project }\end{array}$ & 7.5 & 2.5 \\
\hline 3429 & 2000 & $\begin{array}{l}\text { Public resource management } \\
\text { technical assistance }\end{array}$ & 2.5 & 0.2 \\
\hline 3902 & 2004 & Social sector support project & 6.5 & 5.0 \\
\hline 3993 & 2005 & $\begin{array}{l}\text { Governance Capacity Building } \\
\text { technical assistance }\end{array}$ & 5.0 & 4.0 \\
\hline & & & 78.4 & 12.6 \\
\hline & Of $w h$ & repaid & 4.6 & \\
\hline
\end{tabular}

${ }^{1}$ Less cancellation. 


\section{São Tomé And Príncipe: Relations With the African DeVelopment Bank Group}

1. São Tomé and Príncipe became member of the African Development Bank Group in 1976. To date, the Bank Group has financed twenty-four (24) operations in the country. The net commitments amount to UA 87.56 million, comprising UA 86.56 million of African Development Fund (ADF) ${ }^{14}$ and Technical Assistance Facility (TAF) resources and UA 1.00 million of Nigerian Trust Fund (NTF) resources. The beneficiary sectors of AfDB Group financing are: transport (26.2 percent), multisector ${ }^{15}$ (22.3 percent), agriculture (22.2 percent), social sector (17.9 percent), and public utilities (11.5 percent). These operations have contributed to building and rehabilitating the key basic economic and social infrastructures (roads, airport, electricity, water, schools, health centers), as well as the economic and institutional capacities of the Country. The AfDB has a National Program Office in São Tomé since 1999 and Príncipe.

2. The AfDB's active portfolio is composed of three projects, with an overall disbursement rate of 47 percent (as of August 2005). The three projects, in accordance with the PRSP objectives, have focused on the main factors affecting poverty in STP: institutional and human capacity building, access to water and sanitation, and health, specifically, malaria. The water supply, sanitation and control of water-borne diseases project aims at improving health conditions through an integrated approach, while the poverty reduction project contributes to improve the livelihood of the vulnerable population through targeted actions such as: studies and analysis of poverty profile, census and surveys, statistics capacity building, microinfrastructures, and micro-credit. In other hand, the human resource development project will tackle cross-sectoral capacity building problems by providing for training in the areas of literacy, information, community organization, as well as specialized vocational training, which is suitable for specific demand, particularly in the oil and tourism sectors.

3. The AfDB Group assistance strategy for the 2005-09 period (CSP 2005-09) rests on two key pillars: poverty reduction in rural area and promotion of governance in public finance management. The indicative country allocation on ADF-X resources (2005-07) is UA 5.3 million in form of grants. The first pillar will contribute to promoting and diversifying the economic base of the Country, especially the agricultural and rural sector, and reinforce the operations in the social sectors. The second pillar will focus on supporting macroeconomic reforms and governance,

\footnotetext{
${ }^{14} \mathrm{ADF}$ is the concessional window (or grant for ADF-X for high debt countries — same conditions as IDA—of the African Development Bank Group.

${ }^{15}$ Institutional support and structural adjustment programs.
} 
which will help improve public finance management and institutional capacity building to prepare the forthcoming oil era. Given the considerable needs for institutional capacities building, the AfDB will finance strategic economic and sector studies, such as the Country Governance Profile, the Joint study with the World Bank on the Integrated fiduciary assessment (PER/CFAA/CPAR), and the transport sector study. Furthermore, the AfDB will finance regional capacity building initiatives, within the CEMAC and STP zone, the ECCAS, the African Portuguese-speaking countries and the small insular countries.

4. In addition, the AfDB will continue providing STP with financial assistance through the enhanced HIPC initiative. Within this framework, the AfDB approved in April 2001, a debt relief of US\$ 34.20 million at end-1999 NPV. Due to the delay in the HIPC process, as the completion point is expected to be reached in 2006, the AfDB approved in June 2005 the extension of the interim debt relief period to end2006.

Ongoing projects as of August 31, 2005

(In millions of UA) 1/

\begin{tabular}{lccc}
\hline \multicolumn{1}{c}{ Title of Projects } & $\begin{array}{c}\text { Disbursement } \\
\text { Rate }\end{array}$ & Window & Amounts \\
\hline Poverty reduction & 78 percent & ADF & 1.48 \\
& & TAF & 0.40 \\
\hline $\begin{array}{l}\text { Drinking water supply, Sanitation and Control of } \\
\text { water-borne diseases }\end{array}$ & 63 percent & ADF & 3.20 \\
& & TAF & 1.00 \\
\hline Support for Human resource development & 0 percent & ADF & 3.50 \\
& & ADF & 0.50 \\
& & grant & \\
\hline Total & 47 percent & & 10.08 \\
\hline
\end{tabular}

1/ Exchange rate for September 2005 is UA 1 = US\$1.45984. 


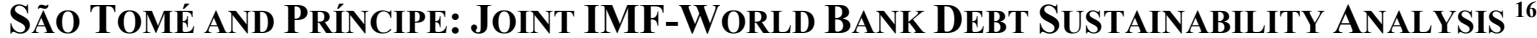

\section{The staffs' assessment of São Tomé and Príncipe's external debt outlook} remains essentially unchanged since the latest World Bank Country Assistance Strategy (IDA/R2005-0087, May 2005) and IMF staff report (IMF Country Report No. 05/323, July 18, 2005). The country faces a high risk of debt distress as the baseline scenario indicates a breach of debt thresholds prior to the production of oil expected to start in early the next decade (Box 1). Indeed, while the attainment of HIPC completion point and the implementation of sound macroeconomic policies will bring about an improvement in the country's debt outlook — particularly in terms of debt service due-São Tomé and Príncipe's external debt stock ratios will remain well above the policy-dependent debt burden thresholds until $2012-13 .{ }^{17}$ The debt stock ratios are highly vulnerable to exchange rate depreciations, a deceleration in export growth, and a reduction in foreign grants and FDI. External debt vulnerabilities (assessed in terms of NPV of debt-to-GDP and NPV of debt-to-revenue ratios) are expected to become more acute under a "no reform scenario," characterized by a lack of fiscal adjustment during the projection period with respect to 2004. The Multilateral Debt Relief Initiative (MDRI), supported by sound macroeconomic policies, would further improve the country's debt indicators starting in 2006.

2. Background information on external debt developments is presented in section I. The evolution of debt since HIPC decision point is briefly discussed in section II. Sections III and IV present the staff's debt sustainability with respect to the relevant policy-dependent thresholds in a scenario that assumes the conduct of prudent macroeconomic policies and the attainment of HIPC completion point in mid-2006. Section V modifies the baseline scenario to include MDRI relief from the Fund and other IFIs. Section VI summarizes the appendix's main findings.

\footnotetext{
16 This debt sustainability analysis for São Tomé and Príncipe was prepared under the Joint World Bank/IMF Debt Sustainability Framework for Low Income Countries of May 2005.

${ }^{17}$ São Tomé and Príncipe is currently classified as a "Poor Performer" according to the World Bank's Country Policy and Institutional Assessment (CPIA) index.
} 


\section{Box 1. São Tomé and Príncipe's Oil Prospects: An Approximation}

- Preliminary staff estimates indicate that, even under the conservative assumption that only one out of the six blocks auctioned in the Joint Development Zone (JDZ) operated with Nigeria is found to be commercially exploitable, oil production would have a sizeable effect on São Tomé and Príncipe's economic prospects.

- The country's oil output share of 30 thousand barrels per day in the JDZ, at a constant price equivalent to US\$30 per barrel, would yield an average of US\$450 million in exports per year (equivalent to approximately 650 percent of the estimated 2006 GDP) for twenty years, and would require a total share of investment of US\$750 million in the country.

- Under this scenario, annual budget revenue from oil could stabilize at around US\$92 million over the long term (equivalent to 130 percent of the estimated 2006 GDP), with the Permanent Fund for Future Generations reaching a steady-state level of US\$3.1 billion in constant 2006 dollar terms.

- The sensitivity analysis shows that oil price changes would not significantly change the staff's assessment about the sustainability of debt once oil production begins.

\section{BACKGROUND}

3. São Tomé and Príncipe's medium- and long-term external debt is estimated at US\$316 million in nominal terms at end-2005, corresponding to a NPV of debt-toexport ratio of 500 percent. The multilateral debt share of total debt is mostly owed to the World Bank and African Development Bank, while the share of official bilateral debt is about 40 percent, of which half is owed to France, Germany, Portugal, the Russian Federation, and Angola. São Tomé and Príncipe is current with its obligations with multilateral creditors and has cleared most of its arrears with Paris Club creditors as part of a new multilateral debt rescheduling agreement on Cologne terms (Box 2). The authorities are currently in the process of reconciling debt records with Paris Club creditors. Contacts with non-Paris Club bilateral creditors are also being pursued to start reconciliation of debt records.

4. HIPC completion point is projected to be reached in mid-2006, provided that the PRGF-supported program has been satisfactorily implemented. Progress toward reaching completion point objectives has been satisfactory so far, especially regarding the preparation of the first annual PRSP report, and meeting its health and education triggers. Satisfactory progress has also been made in improving governance and transparency. ${ }^{18}$

\footnotetext{
${ }^{18}$ See floating completion point triggers in São Tomé and Príncipe HIPC Decision point Document
} 


\section{Box 2. São Tomé and Príncipe: Paris Club Rescheduling}

- On September 13, 2005 Paris Club creditors agreed to provide São Tomé and Príncipe exceptional debt relief during the interim period of the HIPC Initiative. Regarding non-Official Development Assistance, the rescheduling agreement consists of a treatment under Cologne terms of the amounts due of principal and interest (excluding late interest) and not paid from May 1, 2001 to December 31, 2007. Debt repayments on Official Development Assistance (ODA) for the same period are rescheduled over 40-years with 16 years of grace. São Tomé and Príncipe commits to pay 100 percent of the amounts of principal due up to April 30, 2001 inclusive and not paid and of interest accrued up to the same date, inclusive and not paid.

- The interim relief granted by Paris Club creditors will be credited towards total relief at the HIPC Initiative completion point. Regarding non-Paris Club creditors, São Tomé and Príncipe shall inform in writing to the Paris Club no later than April 1, 2006, of the progress made in its negotiations with these creditors; comparability of treatment of debt due would be paramount in this regard.

\section{The Evolution of DebT SINCE HIPC Decision PoInt}

5. São Tomé and Príncipe reached decision point under the Enhanced HIPC Initiative in December 2000 (www.imf.org). In that context, the NPV of debt-toexports ratio was assumed to decline sharply from about 690 percent in 2001 to 143 percent in 2003 and remain at about that level during 2004-08.

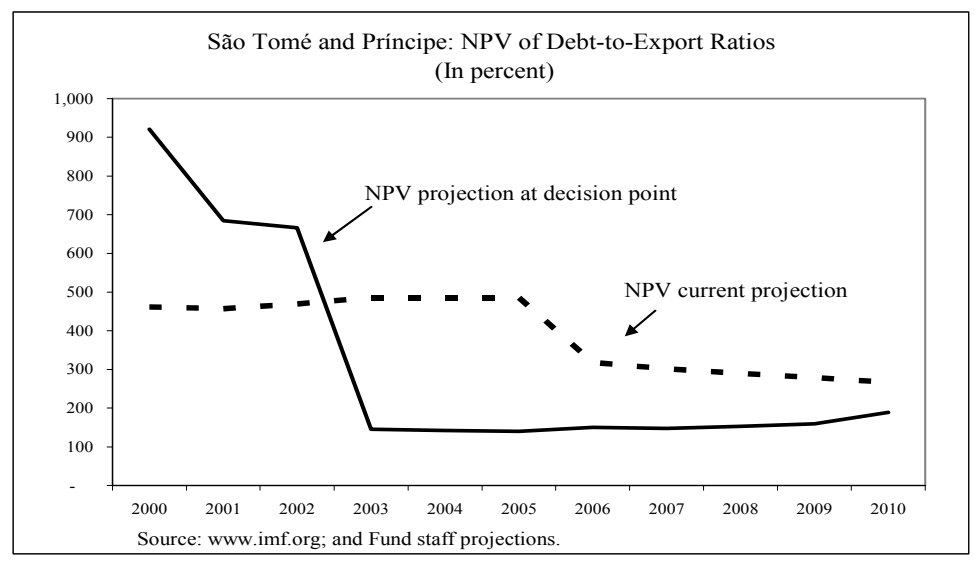

6. Latest staff estimates show significant differences vis-à-vis the levels of NPV of debt-to-exports ratios projected at HIPC decision point. For example, as of end-2006, the level of this ratio is more than twice its original projection. These discrepancies reflect the following factors: ${ }^{19}$

\footnotetext{
${ }^{19}$ An estimation of the relative contribution of each of these factors to the increase in the NPV of debt-to-export ratios could not be done at this point as it would require a loan-by-loan reconciliation. Such an analysis will only be conducted in the context of the full-DSA exercise to be included in the HIPC completion point document.
} 
- A change in the methodology used to calculate the debt indicators from the HIPC DSA framework to the Low Income Countries DSA framework (DSF). These changes are reflected in the definition of exports, the discount rate used to calculate the NPV of debt and the exchange rates used to calculate the debt service projections. $^{20}$

- $\quad$ HIPC completion point is currently assumed for mid-2006. By contrast, the decision point document assumed the attainment of HIPC completion point in 2003.

- Discount rates used to estimate NPV values in the current analysis are lower than those used in 2000 .

- São Tomé and Príncipe's export performance since 2000 has been much weaker than expected.

- $\quad$ There has been some unanticipated new external borrowing, due to a more expansionary fiscal stance than originally envisaged.

- $\quad$ An unanticipated depreciation of the US dollar against the euro since 2000.

\section{Debt Sustainability Analysis}

7. The macroeconomic framework in the staff baseline scenario $(\operatorname{Box} 3)^{21}$ assumes a steady increase in economic activity over the medium term, supported by sound macroeconomic policies and the development of the domestic oil sector. Domestic investment would rise sharply from about 36 percent of GDP to an average of 54.7 percent of GDP in 2008-10, largely on account of oil sector investment initiatives. The rise in the investment ratio will require a continuous reliance on foreign savings, although national savings are forecast to rise on account of an improved financial position of the government and increased private savings once stability and economic growth become firmly established. Key risks to the macro framework arise from a possible weakening of the fiscal consolidation effort in the wake of elections scheduled for 2006. Also, potential shortfalls in foreign assistance triggered by the future oil boom could exacerbate the government's budget constraint in the short run.

\footnotetext{
${ }^{20}$ The DSF uses same-year exports, as opposed to the 3-year backward average used for the decision point. Regarding the discount rates, the DSF methodology uses a fixed 5 percent rate compared to the currencyspecific discount rates under the HIPC methodology. Finally, debt service is projected using WEO assumptions in the DSF, as opposed to fixed (end-1999) exchanges rates used for the decision point. See: "Debt Sustainability in Low-Income Countries: Proposal for an Operational Framework and Policy Implications"www.imf.org and IDA/SecM2004-0035 and "Debt Sustainability in Low-Income CountriesFurther Consideration on an Operational Framework and Policy Implications,"www.imf.org and IDA/SecM2004-0629/1.

${ }^{21}$ The baseline scenario assumes the attainment of HIPC completion point by mid-2006.
} 


\section{Box 3: São Tomé and Príncipe: Macroeconomic Assumptions in Baseline Scenario}

Real GDP growth is projected to average 6 percent in 2005-10 reflecting the implementation of the PRSP and continuous donors' support. The average growth rate is projected to increase to 8 percent in 2011-25 on account of increasing oil production.

Exports of goods and services are projected to increase at an average rate of 10 percent during 2005-10 as a result of strong growth in tourism and a gradual recovery of cocoa export volumes. Oil exports explain the strong export performance projected for 2011-25.

The external current account deficit is expected to average 30 percent of GDP in 2005-10; i.e., roughly unchanged with respect to recent years. The current account is projected to turn into a surplus of about 30 percent of GDP in 2011-25 due mainly to oil exports.

Fiscal projections assumes overall fiscal deficits declining from 18 percent of GDP on average in 2005-06 (excluding oil signature bonuses) to 6 percent in 2010, and a balanced budget thereafter, largely reflecting oil tax receipts.

Multilateral external loan financing is projected at an average of about US\$7.5 million per year during 2005-09, leveling off to US\$6 million in 2010-25.

8. In the baseline scenario, São Tomé and Príncipe's debt outlook shows gradual improvements over the medium term, but it will remain a source of vulnerability until oil production starts in 2012. The NPV of public debt-to-GDP ratio falls from 153 percent in 2005 to 110 in 2006, and to 92 percent in 2010, reflecting a prudent public expenditure envelope underpinning the government's medium-term fiscal strategy (Table 1a). ${ }^{22}$ Only in 2012, the ratio falls significantly to 22 percent of GDP, due mainly to a large increase in GDP associated with the start in oil production. The NPV of debt-to-revenue ratio, at 278 percent in 2005 (Figure 1 and Table 1b), improves significantly in 2006 and remains around 160 percent during 2007-12, before declining gradually thereafter. The debt serviceto-revenue ratio drops significantly following HIPC completion point in 2006 and remains sustainable thereafter.

9. The debt dynamics are particularly vulnerable to shocks. Sensitivity analysis indicates that under the most extreme stress test, defined as a 30 percent depreciation of the dobra exchange rate (measured in U.S. dollar terms), the NPV of debt-to-GDP ratio increases to 186 percent in 2006, compared to about 110 percent in the baseline scenario (see Figure 1 and Table 1b). This reflects the fact that São Tomé and Príncipe's public debt is denominated

\footnotetext{
${ }^{22}$ All public debt is owed to foreign residents (i.e., external public debt).
} 
in foreign currency. The scenario of no-fiscal-reform (defined to include an unchanged primary fiscal balance from its 2004 level, in terms of GDP) brings about an explosive debt burden, underlying the importance of fiscal consolidation to improve the external debt outlook.

\section{EXTERNAL DebT SUSTAINABILITY}

10. The NPV of debt-to-exports ratio improves steadily in the baseline scenario, but will remain significantly above the policy-dependent thresholds until oil production starts. The NPV of debt-to-export ratio decreases from 500 percent in 2005 to 315 percent in 2006, to 271 percent in 2010 (Table 2b). The ratio falls significantly below the 100 percent policy-dependent debt-burden threshold only in 2012, when oil production and exports are projected to begin. The debt service-to-exports ratio declines from 56 percent $^{23}$ in 2005 to about 11 percent during 2007-10, before declining steadily during the following decade, reflecting the delivery of HIPC debt relief and the high concessionality in which new debt is contracted.

11. Sensitivity analysis shows that the NPV of debt-to-exports ratio is particularly vulnerable to a deceleration in export growth. Specifically, a decline in the export growth rate by one standard deviation from historical levels increases the ratio assessed at 300 percent under the baseline scenario to 424 percent in 2007 (Figure 2 and Table 2b).

\section{THE EFFECTS OF MDRI}

12. The implementation of the MDRI would help reduce São Tomé and Príncipe's debt vulnerability (Figure 3, Table 3, and summary table below). The MDRI would reduce the NPV of debt-to-exports ratio from 315 percent in the original baseline scenario to 176 percent in 2006, under the modified baseline; roughly 70 percent higher than the sustainable level in the case of a poor performing country. While NPV ratios would remain above the policy dependent thresholds, the debt service-to-exports ratio would be cut significantly during 2006-10 under the MDRI resulting in substantial savings. The latter reflects the relatively large share of debt to the World Bank and the African Development Bank in the country's total debt.

\footnotetext{
${ }^{23}$ In 2005, debt service refers to debt service due before HIPC completion point and excludes HIPC interim debt relief, rescheduling and cash payments on account of arrears.
} 


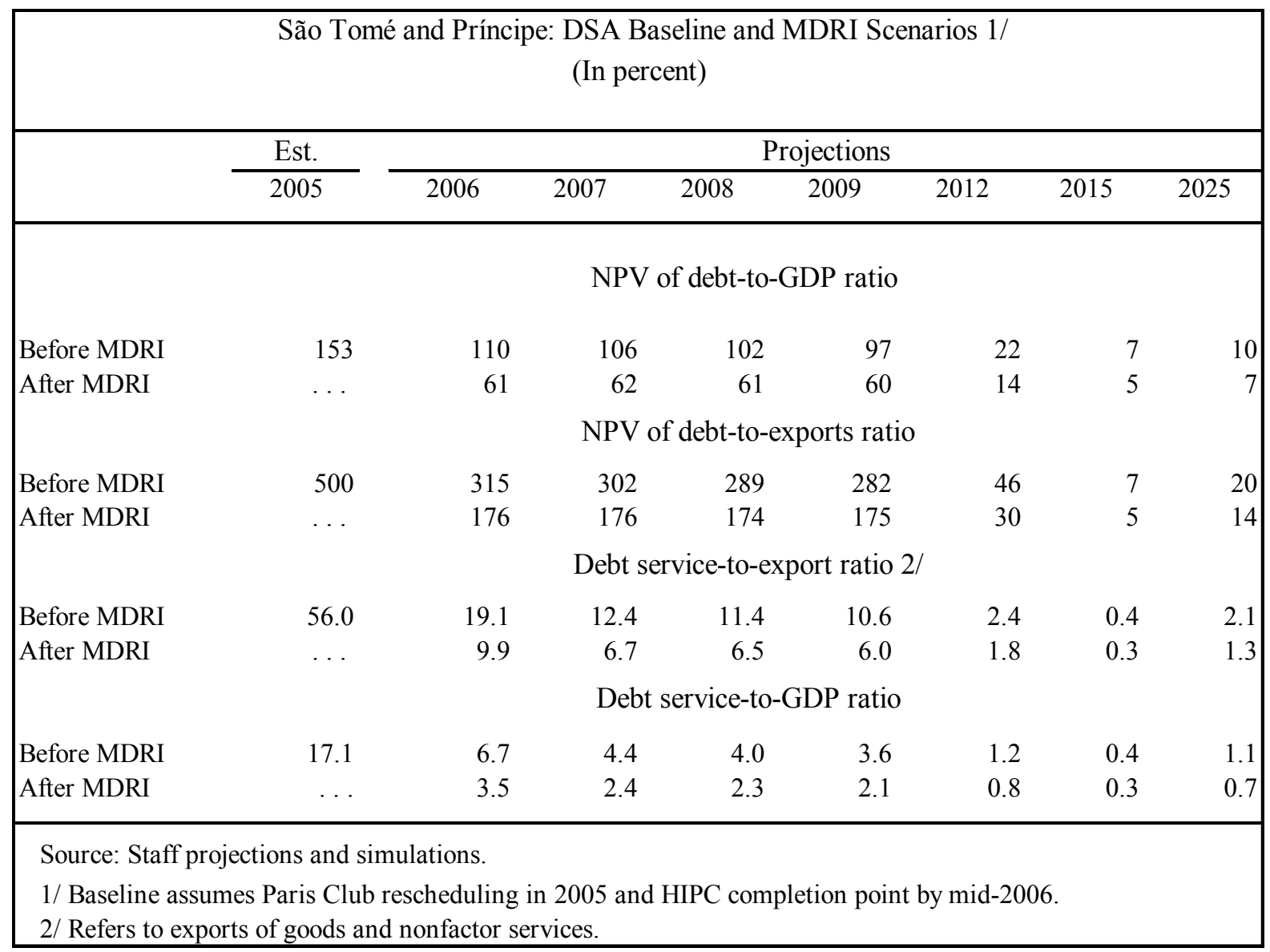

\section{Conclusion}

13. As noted in the latest World Bank Country Assistance Strategy (IDA/R20050087, May 2005) and IMF staff report (IMF Country Report No.05/323, July 18, 2005), the country faces a high risk of debt distress until oil production starts early in the next decade. Also, São Tomé and Príncipe's public debt will remain high and vulnerable to shocks to the exchange rate, exports and foreign grants, at least until oil production starts in 2012/13. Debt vulnerability would be further exacerbated in the absence of sound macroeconomic policies and undue delays with oil production. Calculations indicate that the country would benefit from a significant reduction in debt service under the MDRI starting in 2006. 
Table 1a.São Tomé and Príncipe: Public Sector Debt Sustainability Framework, Baseline Scenario, 2002-2025 (In percent of GDP, unless otherwise indicated)

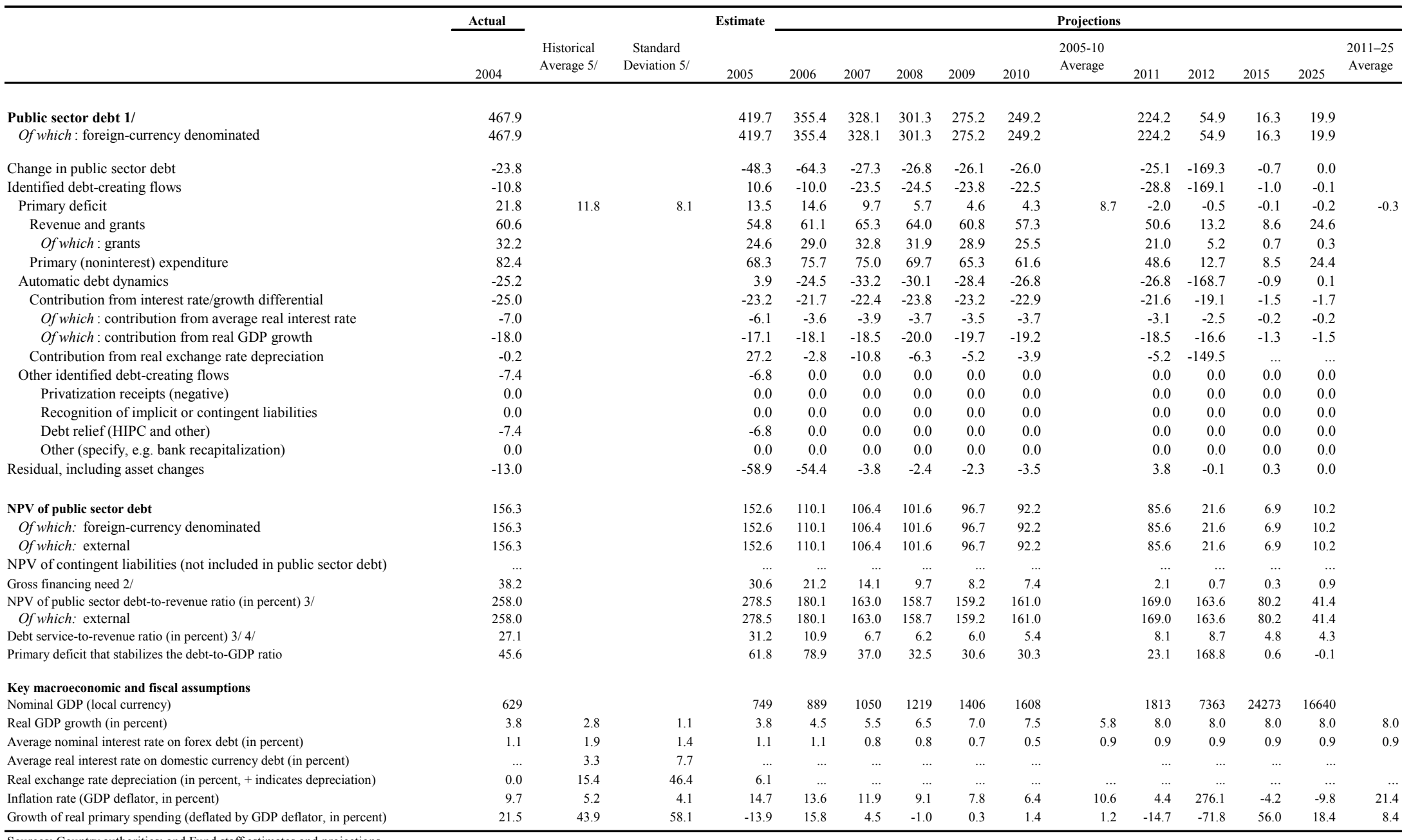

Sources: Country authorities; and Fund staff estimates and projections.

1/ [Indicate coverage of public sector, e.g., general government or nonfinancial public sector. Also whether net or gross debt is used.]

$2 /$ Gross financing need is defined as the primary deficit plus debt service plus the stock of short-term debt at the end of the last period.

3/ Revenues including grants.

4/ Debt service is defined as the sum of interest and amortization of medium and long-term debt.

$5 /$ Historical averages and standard deviations are generally derived over the past 10 years, subject to data availability. 
Table 1b.São Tomé and Príncipe: Sensitivity Analysis for Key Indicators of Public Debt 2005-2025

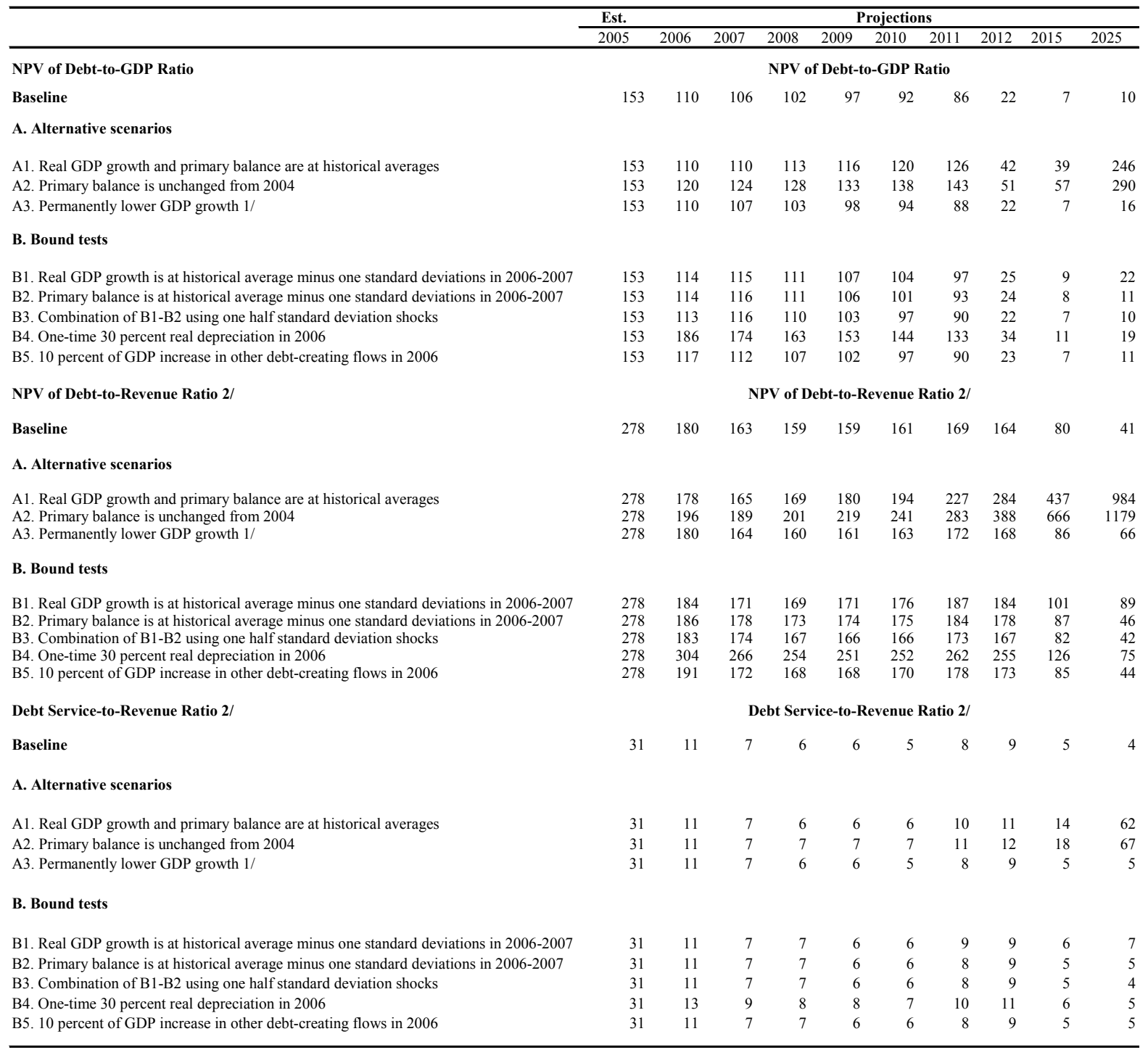

Sources: Country authorities; and Fund staff estimates and projections.

1/ Assumes that real GDP growth is at baseline minus one standard deviation divided by the square root of 20 (i.e., the length of the projection period).

$2 /$ Revenues are defined inclusive of grants. 
Fig. 1.São Tomé and Príncipe: Indicators of Public Debt Under Alternative Scenarios, 2005-2025

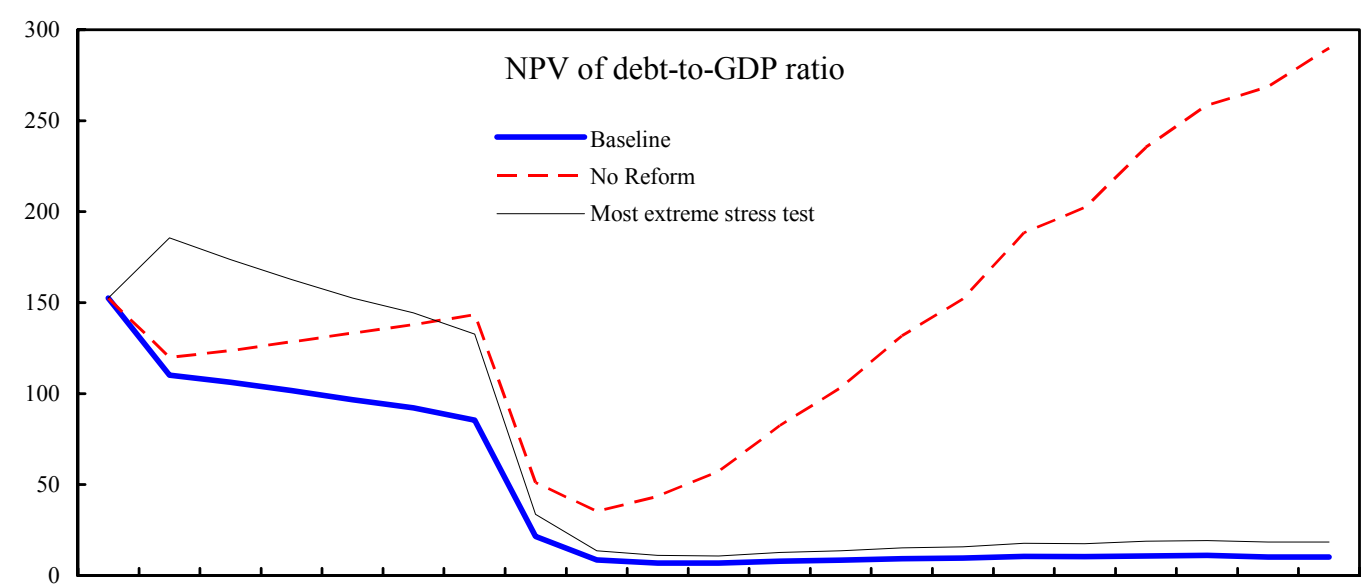

200520062007200820092010201120122013201420152016201720182019202020212022202320242025

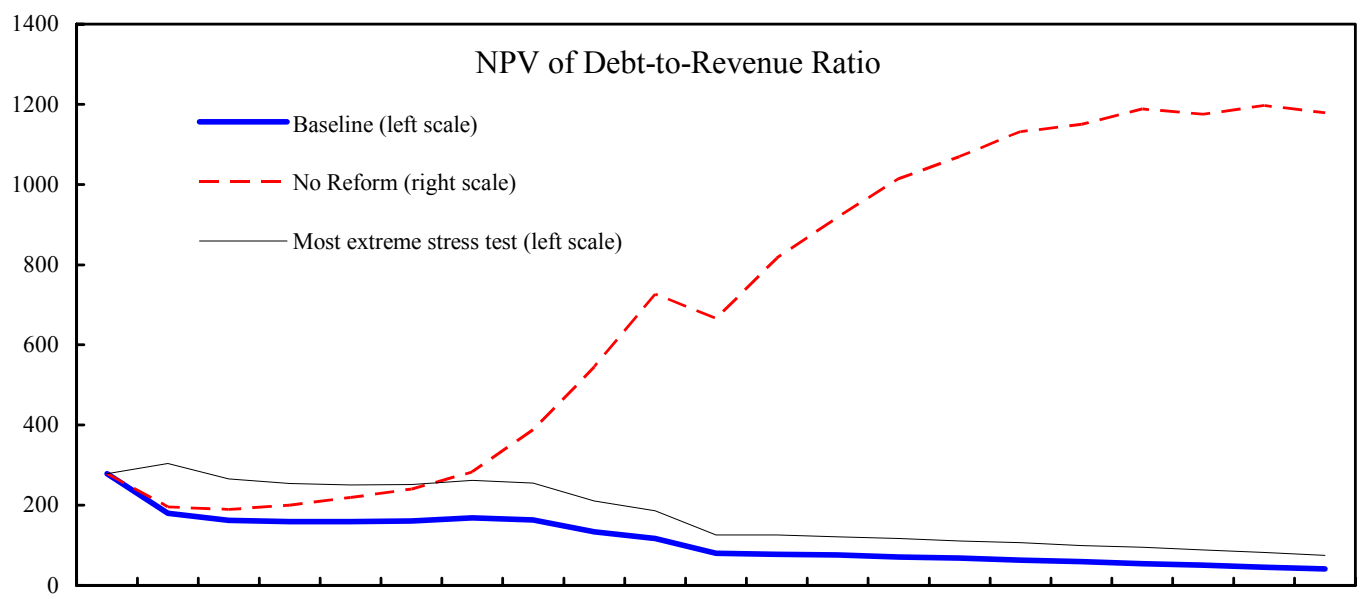

200520062007200820092010201120122013201420152016201720182019202020212022202320242025

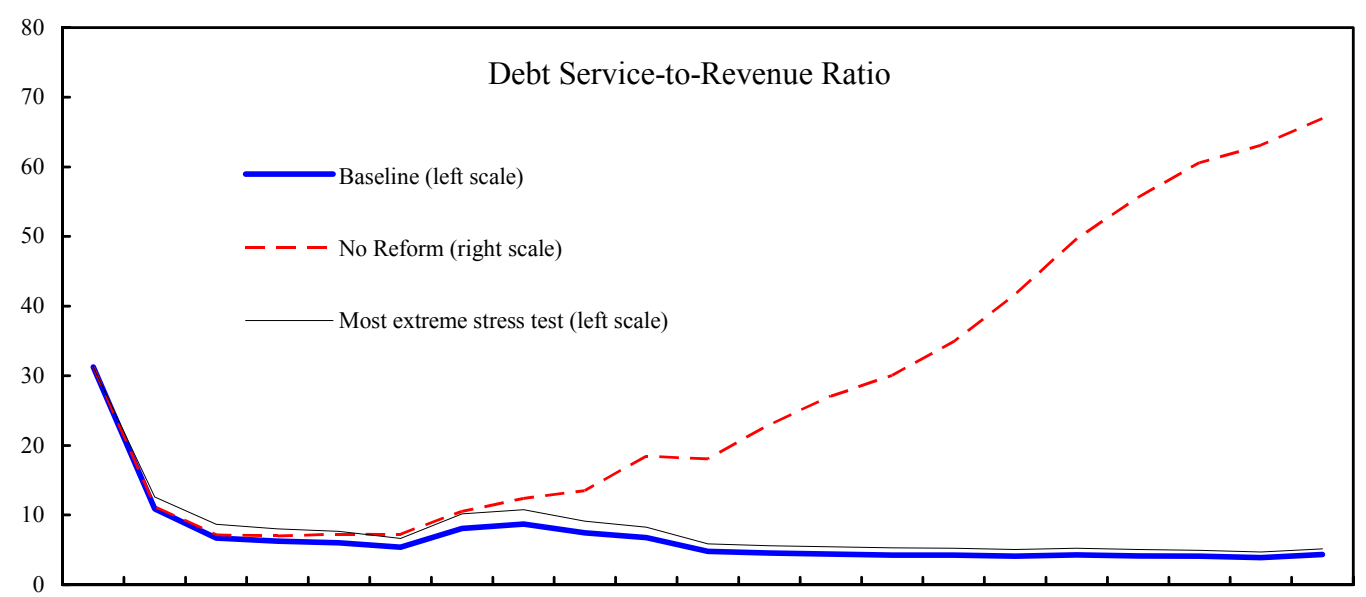

200520062007200820092010201120122013201420152016201720182019202020212022202320242025

Source: Staff projections.

CInternational Monetary Fund. Not for Redistribution 
Table 2a. São Tomé and Príncipe: External Debt Sustainability Framework, Baseline Scenario, 2005-2025 1/

(In percent of GDP, unless otherwise indicated)

\begin{tabular}{|c|c|c|c|c|c|c|c|c|c|c|c|c|c|c|}
\hline & \multirow[b]{2}{*}{$\begin{array}{rl}\text { Est. } & \\
2005 & 0\end{array}$} & \multirow[b]{2}{*}{$\begin{array}{c}\text { Historical } \\
\text { Average 5/ }\end{array}$} & \multirow[b]{2}{*}{$\begin{array}{c}\text { Standard } \\
\text { Deviation 5/ }\end{array}$} & \multicolumn{10}{|c|}{ Projections } & \multirow[b]{2}{*}{$\begin{array}{r}2011-25 \\
\text { Average } \\
\end{array}$} \\
\hline & & & & 2006 & 2007 & 2008 & 2009 & 2010 & $\begin{array}{l}2005-10 \\
\text { Average }\end{array}$ & 2011 & 2012 & 2015 & 2025 & \\
\hline External debt (nominal) $1 /$ & 419.7 & & & 355.4 & 328.1 & 301.3 & 275.2 & 249.2 & & 224.2 & 54.9 & 16.3 & 19.9 & \\
\hline Of which: public and publicly guaranteed (PPG) & 419.7 & & & 355.4 & 328.1 & 301.3 & 275.2 & 249.2 & & 224.2 & 54.9 & 16.3 & 19.9 & \\
\hline Change in external debt & -48.3 & & & -64.3 & -27.3 & -26.8 & -26.1 & -26.0 & & -25.1 & -169.3 & -0.7 & 0.0 & \\
\hline Identified net debt-creating flows & -11.6 & & & 4.2 & 6.2 & 7.0 & 5.9 & 5.1 & & 67.3 & 75.0 & -39.6 & 10.7 & \\
\hline Non-interest current account deficit & 28.6 & 16.9 & 10.2 & 24.3 & 26.9 & 29.6 & 28.5 & 27.9 & & 103.3 & 84.1 & -36.6 & 16.3 & -2.6 \\
\hline Deficit in balance of goods and services & 57.8 & & & 59.4 & 61.7 & 64.9 & 61.0 & 56.8 & & 127.9 & 30.0 & -71.4 & -9.9 & \\
\hline Exports & 30.5 & & & 34.9 & 35.2 & 35.2 & 34.3 & 34.1 & & 34.7 & 47.0 & 93.4 & 50.1 & \\
\hline Imports & 88.4 & & & 94.3 & 97.0 & 100.1 & 95.3 & 90.8 & & 162.7 & 77.0 & 22.0 & 40.2 & \\
\hline Net current transfers (negative $=$ inflow) & -29.0 & -40.2 & 13.5 & -34.4 & -35.3 & -34.6 & -32.1 & -29.0 & & -24.7 & -5.9 & -1.0 & -1.0 & -3.0 \\
\hline Other current account flows (negative $=$ net inflow) & -0.3 & & & -0.7 & 0.5 & -0.6 & -0.4 & 0.2 & & 0.0 & 60.0 & 35.7 & 27.2 & \\
\hline Net FDI (negative = inflow) & -5.0 & -6.1 & 2.4 & -5.4 & -5.4 & -5.5 & -5.5 & -5.5 & & -20.1 & -5.2 & -1.8 & -4.2 & -4.2 \\
\hline Endogenous debt dynamics 2/ & -35.1 & & & -14.7 & -15.3 & -17.2 & -17.1 & -17.3 & & -15.9 & -3.9 & -1.2 & -1.4 & \\
\hline Contribution from nominal interest rate & 4.5 & & & 4.0 & 2.5 & 2.1 & 1.9 & 1.3 & & 2.0 & 0.5 & 0.1 & 0.2 & \\
\hline Contribution from real GDP growth & -16.3 & & & -18.7 & -17.8 & -19.4 & -19.1 & -18.6 & & -17.9 & -4.4 & -1.3 & -1.6 & \\
\hline Contribution from price and exchange rate changes & -23.4 & & & & & & & $\ldots$ & & $\ldots$ & & & $\ldots$ & \\
\hline Residual (3-4) 3/ & -36.7 & & & -68.6 & -33.4 & -33.8 & -32.0 & -31.1 & & -92.4 & -244.3 & 38.9 & -10.7 & \\
\hline NPV of external debt & 152.6 & & & 110.1 & 106.4 & 101.6 & 96.7 & 92.2 & & 85.6 & 21.6 & 6.9 & 10.2 & \\
\hline In percent of exports & 499.5 & & & 315.5 & 301.9 & 288.7 & 282.1 & 270.8 & & 246.4 & 45.9 & 7.4 & 20.3 & \\
\hline NPV of PPG external debt & 152.6 & & & 110.1 & 106.4 & 101.6 & 96.7 & 92.2 & & 85.6 & 21.6 & 6.9 & 10.2 & \\
\hline In percent of exports & 499.5 & & & 315.5 & 301.9 & 288.7 & 282.1 & 270.8 & & 246.4 & 45.9 & 7.4 & 20.3 & \\
\hline Debt service-to-exports ratio (in percent) & 56.0 & & & 19.1 & 12.4 & 11.4 & 10.6 & 9.1 & & 11.8 & 2.4 & 0.4 & 2.1 & \\
\hline PPG debt service-to-exports ratio (in percent) & 56.0 & & & 19.1 & 12.4 & 11.4 & 10.6 & 9.1 & & 11.8 & 2.4 & 0.4 & 2.1 & \\
\hline Total gross financing need (billions of U.S. dollars) & 0.0 & & & 0.0 & 0.0 & 0.0 & 0.0 & 0.0 & & 0.1 & 0.4 & -0.6 & 0.1 & \\
\hline Non-interest current account deficit that stabilizes debt ratio & 76.8 & & & 88.6 & 54.2 & 56.5 & 54.6 & 53.9 & & 128.3 & 253.3 & -35.9 & 16.3 & \\
\hline \multicolumn{15}{|l|}{ Key macroeconomic assumptions } \\
\hline Real GDP growth (in percent) & 3.8 & 3.0 & 1.1 & 4.5 & 5.5 & 6.5 & 7.0 & 7.5 & 6.2 & 8.0 & 8.0 & 8.0 & 8.0 & 8.0 \\
\hline GDP deflator in US dollar terms (change in percent) & 5.3 & 1.2 & 7.1 & -3.3 & 4.1 & 3.4 & 3.4 & 3.1 & 2.1 & 3.2 & 277.2 & -4.0 & -9.5 & 21.6 \\
\hline Effective interest rate (percent) 4 / & 1.0 & 1.2 & 0.5 & 1.0 & 0.8 & 0.7 & 0.7 & 0.5 & 0.7 & 0.9 & 0.9 & 0.9 & 0.9 & 0.9 \\
\hline Growth of exports of G\&S (US dollar terms, in percent) & 6.9 & 9.3 & 12.6 & 15.4 & 10.9 & 10.0 & 7.7 & 10.1 & 10.8 & 13.6 & 451.6 & 2.4 & -14.7 & 54.3 \\
\hline Growth of imports of G\&S (US dollar terms, in percent) & 9.0 & 4.4 & 16.6 & 7.8 & 12.9 & 13.7 & 5.3 & 5.6 & 9.1 & 99.5 & 92.9 & -27.3 & 10.1 & 17.1 \\
\hline Grant element of new public sector borrowing (including IMF; in percent) & $\ldots$ & $\ldots$ & $\ldots$ & 42.4 & 44.0 & 47.1 & 48.9 & 46.4 & 45.8 & 48.1 & 48.1 & 48.1 & 48.1 & 48.1 \\
\hline Grant element of new public sector borrowing (excluding IMF; in percent) & $\ldots$ & $\ldots$ & $\ldots$ & 46.9 & 47.6 & 49.3 & 48.9 & 46.4 & 47.8 & 48.1 & 48.1 & 48.1 & 48.1 & 48.1 \\
\hline Memorandum item: & & & & & & & & & & & & & & \\
\hline Nominal GDP (billions of US dollars) & 0.1 & & & 0.1 & 0.1 & 0.1 & 0.1 & 0.1 & & 0.1 & 0.5 & 1.6 & 1.1 & \\
\hline
\end{tabular}

Source: Staff projections.

1/ External public debt only.

2/ Derived as $[\mathrm{r}-\mathrm{g}-\mathrm{r}(1+\mathrm{g})](1+\mathrm{g}+\mathrm{r}+\mathrm{gr})$ times previous period debt ratio, with $\mathrm{r}=$ nominal interest rate; $\mathrm{g}=$ real GDP growth rate, and $\mathrm{r}=$ growth rate of GDP deflator in U.S. dollar terms.

3/ Includes exceptional financing (i.e., changes in arrears and debt relief); changes in gross foreign assets; and valuation adjustments. For projections also includes contribution from price and exchange rate changes.

4/ Current-year interest payments devided by previous period debt stock.

5 / Historical averages and standard deviations are generally derived over the past 10 years, subject to data availability. 
Table 2b. São Tomé and Príncipe: Sensitivity Analyses for Key Indicators of Public and Publicly Guaranteed External Debt, 2005-2025 (In percent)

\begin{tabular}{|c|c|c|c|c|c|c|c|c|c|c|}
\hline & \multirow{2}{*}{$\begin{array}{r}\text { Est. } \\
2005\end{array}$} & \multicolumn{9}{|c|}{ Projections } \\
\hline & & 2006 & 2007 & 2008 & 2009 & 2010 & 2011 & 2012 & 2015 & 2025 \\
\hline & \multicolumn{10}{|c|}{ NPV of debt-to-GDP ratio $1 /$} \\
\hline Baseline & 153 & 110 & 106 & 102 & 97 & 92 & 86 & 22 & 7 & 10 \\
\hline \multicolumn{11}{|l|}{ A. Alternative Scenarios } \\
\hline A1. Key variables at their historical averages in $2006-252 /$ & 153 & 102 & 103 & 102 & 104 & 106 & 68 & 69 & 132 & 73 \\
\hline A2. New public sector loans on less favorable terms in 2006-25 3/ & 153 & 110 & 108 & 105 & 101 & 98 & 92 & 23 & 8 & 13 \\
\hline A3. MDRI & $\ldots$ & 61 & 62 & 61 & 60 & 59 & 55 & 14 & 5 & 7 \\
\hline \multicolumn{11}{|l|}{ B. Bound Tests } \\
\hline B1. Real GDP growth at historical average minus one standard deviation in 2006-07 & 153 & 114 & 114 & 109 & 103 & 99 & 91 & 23 & 7 & 11 \\
\hline B2. Export value growth at historical average minus one standard deviation in 2006-07 4/ & 153 & 113 & 109 & 104 & 99 & 94 & 87 & 22 & 7 & 9 \\
\hline B3. US dollar GDP deflator at historical average minus one standard deviation in 2006-07 & 153 & 114 & 122 & 116 & 111 & 106 & 98 & 25 & 8 & 12 \\
\hline B4. Net non-debt creating flows at historical average minus one standard deviation in 2006-07 5/ & 153 & 115 & 111 & 106 & 100 & 96 & 89 & 22 & 7 & 10 \\
\hline B5. Combination of B1-B4 using one-half standard deviation shocks & 153 & 114 & 121 & 115 & 109 & 104 & 97 & 24 & 8 & 10 \\
\hline \multirow[t]{2}{*}{ B6. One-time 30 percent nominal depreciation relative to the baseline in 2006 6/ } & 153 & 160 & 154 & 159 & 151 & 144 & 134 & 34 & 11 & 15 \\
\hline & \multicolumn{10}{|c|}{ NPV of debt-to-exports ratio } \\
\hline Baseline & 500 & 315 & 302 & 289 & 282 & 271 & 246 & 46 & 7 & 20 \\
\hline \multicolumn{11}{|l|}{ A. Alternative Scenarios } \\
\hline A1. Key variables at their historical averages in $2006-252 /$ & 500 & 301 & 293 & 291 & 303 & 310 & 197 & 146 & 141 & 145 \\
\hline A2. New public sector loans on less favorable terms in $2006-25 \mathrm{3} /$ & 500 & 315 & 307 & 298 & 296 & 287 & 265 & 50 & 8 & 27 \\
\hline A3. MDRI & $\cdots$ & 176 & 176 & 174 & 175 & 172 & 158 & 30 & 5 & 14 \\
\hline \multicolumn{11}{|l|}{ B. Bound Tests } \\
\hline B1. Real GDP growth at historical average minus one standard deviation in 2006-07 & 500 & 326 & 304 & 291 & 284 & 273 & 248 & 46 & 7 & 20 \\
\hline B2. Export value growth at historical average minus one standard deviation in 2006-07 4/ & 500 & 386 & 424 & 405 & 395 & 379 & 344 & 64 & 10 & 25 \\
\hline B3. US dollar GDP deflator at historical average minus one standard deviation in 2006-07 & 500 & 327 & 304 & 291 & 284 & 273 & 248 & 46 & 7 & 20 \\
\hline B4. Net non-debt creating flows at historical average minus one standard deviation in 2006-07 5/ & 500 & 329 & 314 & 300 & 293 & 281 & 256 & 48 & 8 & 19 \\
\hline B5. Combination of B1-B4 using one-half standard deviation shocks & 500 & 362 & 372 & 356 & 347 & 333 & 303 & 56 & 9 & 22 \\
\hline \multirow[t]{2}{*}{ B6. One-time 30 percent nominal depreciation relative to the baseline in 2006 6/ } & 500 & 457 & 302 & 312 & 304 & 292 & 266 & 49 & 8 & 21 \\
\hline & \multicolumn{10}{|c|}{ Debt service-to-export ratio } \\
\hline Baseline & 56.0 & 19.1 & 12.4 & 11.4 & 10.6 & 9.1 & 11.8 & 2.4 & 0.4 & 2.1 \\
\hline \multicolumn{11}{|l|}{ A. Alternative Scenarios } \\
\hline A1. Key variables at their historical averages in $2006-252 /$ & 56.0 & 19.1 & 12.4 & 11.8 & 11.5 & 9.0 & 13.2 & 10.0 & 4.4 & 24.3 \\
\hline A2. New public sector loans on less favorable terms in $2006-253$ / & 56.0 & 18.9 & 12.3 & 11.6 & 11.2 & 9.1 & 10.6 & 2.1 & 0.4 & 2.3 \\
\hline A3. MDRI & $\ldots$ & 9.9 & 6.7 & 6.5 & 6.0 & 5.4 & 8.8 & 1.8 & 0.3 & 1.3 \\
\hline \multicolumn{11}{|l|}{ B. Bound Tests } \\
\hline B1. Real GDP growth at historical average minus one standard deviation in 2006-07 & 56.0 & 19.6 & 12.3 & 11.3 & 10.5 & 8.1 & 10.9 & 2.3 & 0.4 & 2.1 \\
\hline B2. Export value growth at historical average minus one standard deviation in 2006-07 4/ & 56.0 & 22.8 & 17.0 & 15.7 & 14.9 & 11.5 & 15.3 & 3.2 & 0.7 & 3.1 \\
\hline B3. US dollar GDP deflator at historical average minus one standard deviation in 2006-07 & 56.0 & 19.6 & 12.3 & 11.3 & 10.5 & 8.1 & 10.9 & 2.3 & 0.4 & 2.1 \\
\hline B4. Net non-debt creating flows at historical average minus one standard deviation in 2006-07 5/ & 56.0 & 19.1 & 12.4 & 11.5 & 10.8 & 8.3 & 11.1 & 2.3 & 0.5 & 2.2 \\
\hline B5. Combination of B1-B4 using one-half standard deviation shocks & 56.0 & 21.4 & 14.9 & 13.9 & 13.1 & 10.1 & 13.4 & 2.8 & 0.6 & 2.7 \\
\hline B6. One-time 30 percent nominal depreciation relative to the baseline in 2006 6/ & 56.0 & 27.7 & 12.6 & 11.5 & 10.8 & 8.3 & 11.1 & 2.3 & 0.5 & 2.2 \\
\hline \multicolumn{11}{|l|}{ Memorandum item: } \\
\hline Grant element assumed on residual financing (i.e., financing required above baseline) $7 /$ & 53 & 51 & 49 & 47 & 45 & 43 & 40 & 38 & 36 & 32 \\
\hline
\end{tabular}

1/ By the year 2023 real GDP growth slows down significantly due to the projected decline in petroleum production.

2/ Variables include real GDP growth, growth of GDP deflator (in U.S. dollar terms), non-interest current account in percent of GDP, and non-debt creating flows.

3 / Assumes that the interest rate on new borrowing is by 2 percentage points higher than in the baseline., while grace and maturity periods are the same as in the baseline.

4/ Exports values are assumed to remain permanently at the lower level, but the current account as a share of GDP is assumed to return to its baseline level after the shock

(implicitly assuming an offsetting adjustment in import levels).

$5 /$ Includes official and private transfers and FDI

6/ Depreciation is defined as percentage decline in dollar/local currency rate, such that it never exceeds 100 percent.

7/ Based on historical level of concessionality. Applies to all stress scenarios except for A2 (less favorable financing) in which the terms on all new financing are as specified in footnote 2 .

\section{CInternational Monetary Fund. Not for Redistribution}


Fig. 2. São Tomé and Príncipe: Indicators of External Debt, 2005-25

(In percent)
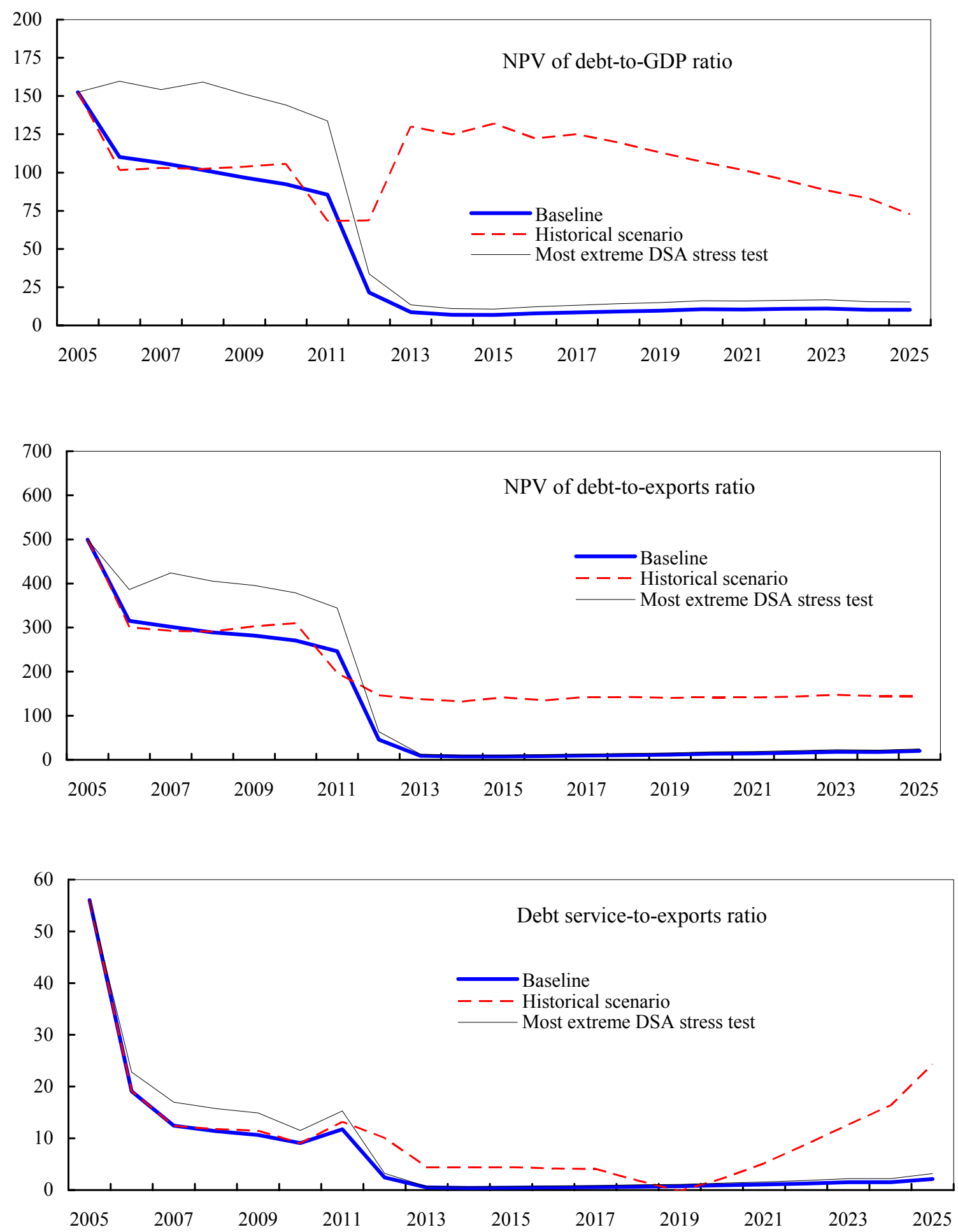

Source: Staff projections. 
Fig. 3. São Tomé and Príncipe: External Debt under the Baseline and MDRI, 2005-2025 (In percent)
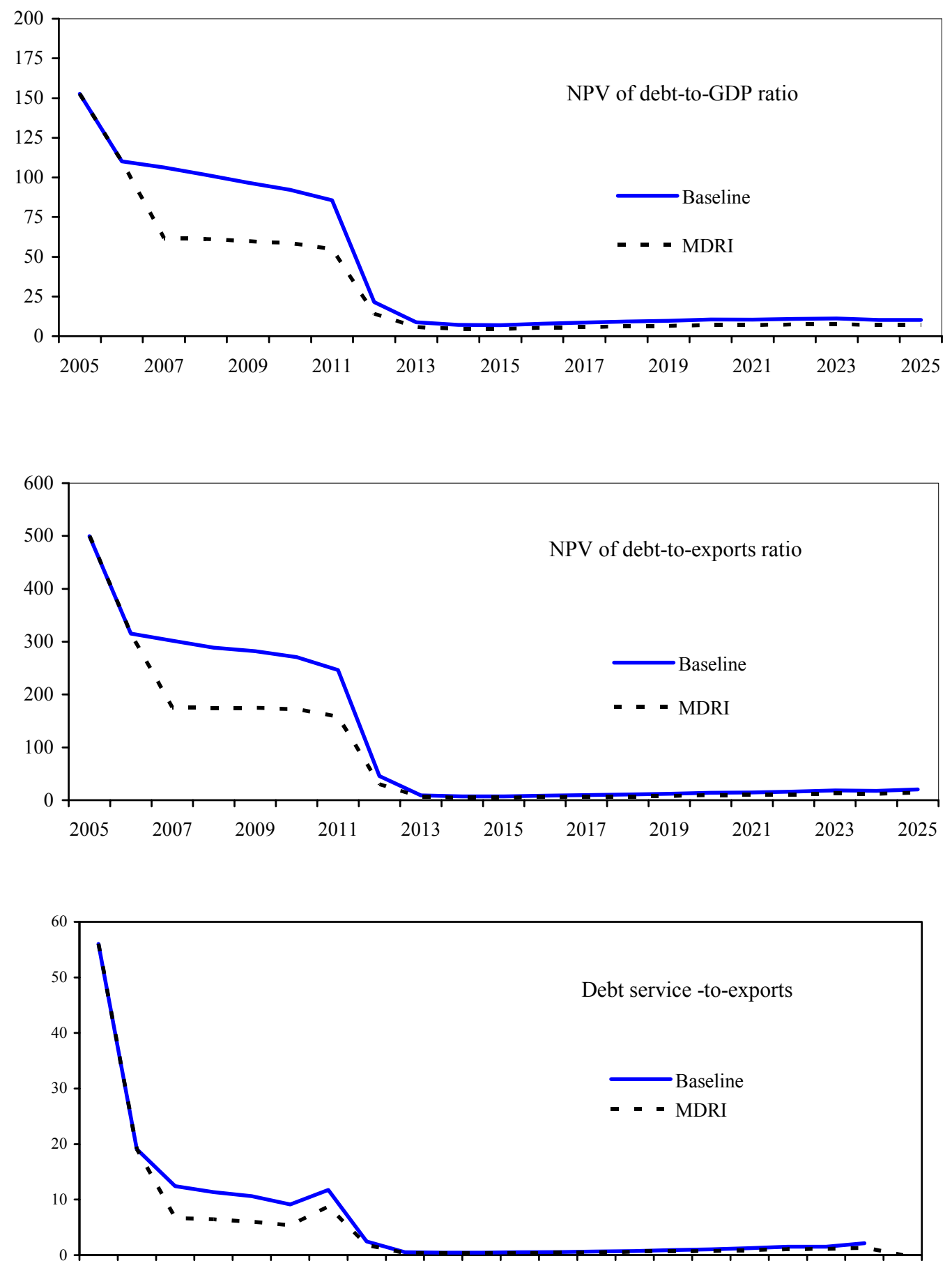

200520062007200820092010201120122013201420152016201720182019202020212022202320242025

Source: Staff projections. 
Table 3. São Tomé and Príncipe: Sensitivity Analyses for Key Indicators of Public and Publicly Guaranteed External Debt under DMRI, 2005-2025 (In percent)

\begin{tabular}{|c|c|c|c|c|c|c|c|c|c|c|}
\hline & \multirow{2}{*}{$\begin{array}{r}\text { Est. } \\
2005 \\
\end{array}$} & \multicolumn{9}{|c|}{ Projections } \\
\hline & & 2006 & 2007 & 2008 & 2009 & 2010 & 2011 & 2012 & 2015 & 2025 \\
\hline & \multicolumn{10}{|c|}{ NPV of debt-to-GDP ratio 1 / } \\
\hline Baseline under DMRI & 153 & 61 & 62 & 61 & 60 & 59 & 55 & 14 & 5 & 7 \\
\hline \multicolumn{11}{|l|}{ A. Alternative Scenarios } \\
\hline A1. Key variables at their historical averages in 2006-25 2/ & 153 & 50 & 57 & 58 & 61 & 64 & 28 & 27 & 94 & 50 \\
\hline A2. New public sector loans on less favorable terms in $2006-253$ / & 153 & 61 & 64 & 65 & 65 & 64 & 61 & 16 & 5 & 10 \\
\hline A3. MDRI & $\ldots$ & 66 & 67 & 66 & 64 & 62 & 58 & 15 & 5 & 7 \\
\hline \multicolumn{11}{|l|}{ B. Bound Tests } \\
\hline B1. Real GDP growth at historical average minus one standard deviation in 2006-07 & 153 & 67 & 70 & 69 & 67 & 66 & 61 & 16 & 5 & 8 \\
\hline B2. Export value growth at historical average minus one standard deviation in 2006-07 4/ & 153 & 64 & 64 & 64 & 62 & 61 & 57 & 14 & 5 & 6 \\
\hline B3. US dollar GDP deflator at historical average minus one standard deviation in 2006-07 & 153 & 67 & 75 & 74 & 72 & 71 & 66 & 17 & 5 & 8 \\
\hline B4. Net non-debt creating flows at historical average minus one standard deviation in 2006-07 5/ & 153 & 66 & 66 & 65 & 64 & 62 & 58 & 15 & 5 & 7 \\
\hline B5. Combination of B1-B4 using one-half standard deviation shocks & 153 & 66 & 72 & 71 & 70 & 68 & 64 & 16 & 5 & 7 \\
\hline \multirow[t]{2}{*}{ B6. One-time 30 percent nominal depreciation relative to the baseline in 2006 6/ } & 153 & 89 & 90 & 148 & 143 & 138 & 129 & 33 & 11 & 13 \\
\hline & \multicolumn{10}{|c|}{ NPV of debt-to-exports ratio } \\
\hline Baseline under DMRI & 500 & 176 & 176 & 174 & 175 & 172 & 158 & 30 & 5 & 14 \\
\hline \multicolumn{11}{|l|}{ A. Alternative Scenarios } \\
\hline A1. Key variables at their historical averages in 2006-2025 2/ & 500 & 148 & 163 & 166 & 178 & 188 & 79 & 58 & 101 & 100 \\
\hline A2. New public sector loans on less favorable terms in $2006-253$ / & 500 & 176 & 181 & 183 & 189 & 189 & 177 & 34 & 6 & 21 \\
\hline A3. MDRI & $\cdots$ & 190 & 189 & 187 & 187 & 183 & 167 & 31 & 5 & 14 \\
\hline \multicolumn{11}{|l|}{ B. Bound Tests } \\
\hline B1. Real GDP growth at historical average minus one standard deviation in 2006-07 & 500 & 192 & 186 & 184 & 184 & 181 & 167 & 31 & 5 & 14 \\
\hline B2. Export value growth at historical average minus one standard deviation in 2006-07 4/ & 500 & 220 & 251 & 248 & 248 & 244 & 224 & 42 & 7 & 17 \\
\hline B3. US dollar GDP deflator at historical average minus one standard deviation in 2006-07 & 500 & 193 & 187 & 185 & 185 & 182 & 167 & 31 & 5 & 15 \\
\hline B4. Net non-debt creating flows at historical average minus one standard deviation in 2006-07 5/ & 500 & 189 & 188 & 185 & 186 & 182 & 167 & 31 & 5 & 13 \\
\hline B5. Combination of B1-B4 using one-half standard deviation shocks & 500 & 209 & 224 & 221 & 221 & 217 & 199 & 37 & 6 & 15 \\
\hline \multirow[t]{2}{*}{ B6. One-time 30 percent nominal depreciation relative to the baseline in 2006 6/ } & 500 & 255 & 176 & 291 & 287 & 278 & 255 & 48 & 8 & 18 \\
\hline & \multicolumn{10}{|c|}{ Debt service-to-export ratio } \\
\hline Baseline under DMRI & 56.0 & 9.9 & 6.7 & 6.5 & 6.0 & 5.4 & 8.8 & 1.8 & 0.3 & 1.3 \\
\hline \multicolumn{11}{|l|}{ A. Alternative Scenarios } \\
\hline A1. Key variables at their historical averages in 2006-2025 2/ & 56.0 & 13.0 & 6.3 & 5.5 & 6.0 & 4.1 & 9.1 & 6.6 & 2.3 & 15.6 \\
\hline A2. New public sector loans on less favorable terms in $2006-20253 /$ & 56.0 & 12.8 & 6.5 & 5.9 & 6.7 & 5.3 & 7.6 & 1.4 & 0.3 & 1.5 \\
\hline A3. MDRI & $\cdots$ & 9.9 & 6.7 & 6.5 & 6.0 & 5.4 & 8.8 & 1.8 & 0.3 & 1.3 \\
\hline \multicolumn{11}{|l|}{ B. Bound Tests } \\
\hline B1. Real GDP growth at historical average minus one standard deviation in 2006-07 & 56.0 & 13.3 & 6.6 & 5.7 & 6.0 & 4.3 & 7.9 & 1.7 & 0.3 & 1.4 \\
\hline B2. Export value growth at historical average minus one standard deviation in 2006-07 4/ & 56.0 & 15.5 & 9.0 & 7.9 & 8.7 & 6.2 & 11.2 & 2.3 & 0.5 & 2.0 \\
\hline B3. US dollar GDP deflator at historical average minus one standard deviation in 2006-07 & 56.0 & 13.4 & 6.7 & 5.7 & 6.1 & 4.3 & 7.9 & 1.7 & 0.3 & 1.4 \\
\hline B4. Net non-debt creating flows at historical average minus one standard deviation in 2006-07 5/ & 56.0 & 13.0 & 6.7 & 5.9 & 6.2 & 4.4 & 8.1 & 1.7 & 0.4 & 1.4 \\
\hline B5. Combination of B1-B4 using one-half standard deviation shocks & 56.0 & 14.6 & 8.0 & 7.1 & 7.6 & 5.5 & 9.8 & 2.0 & 0.5 & 1.8 \\
\hline B6. One-time 30 percent nominal depreciation relative to the baseline in 2006 6/ & 56.0 & 18.9 & 8.1 & 7.1 & 7.3 & 5.5 & 9.0 & 1.8 & 0.6 & 2.0 \\
\hline \multicolumn{11}{|l|}{ Memorandum item: } \\
\hline Grant element assumed on residual financing (i.e., financing required above baseline) 7/ & 53 & 51 & 49 & 47 & 45 & 43 & 40 & 38 & 36 & 32 \\
\hline
\end{tabular}

Source: Staff projections.

1/ By the year 2023 real GDP growth slows down significantly due to the projected decline in petroleum production.

2/ Variables include real GDP growth, growth of GDP deflator (in U.S. dollar terms), non-interest current account in percent of GDP, and non-debt creating flows.

3 / Assumes that the interest rate on new borrowing is by 2 percentage points higher than in the baseline., while grace and maturity periods are the same as in the baseline.

4/ Exports values are assumed to remain permanently at the lower level, but the current account as a share of GDP is assumed to return to its baseline level after the shock (implicitly assuming an offsetting adjustment in import levels).

5/ Includes official and private transfers and FDI.

6/ Depreciation is defined as percentage decline in dollar/local currency rate, such that it never exceeds 100 percent.

7/ Based on historical level of concessionality. Applies to all stress scenarios except for A2 (less favorable financing) in which the terms on all new financing

are as specified in footnote 2 .

\section{CInternational Monetary Fund. Not for Redistribution}




\section{São Tomé and Príncipe: Statistical Issues}

\section{Introduction}

21. Although economic data are generally adequate for surveillance, serious shortcomings persist despite the efforts made and the technical assistance provided. São Tomé and Príncipe's statistical system is still developing and suffers from serious financial, human and technological resource constraints, which have slowed down efforts to strengthen the system.

22. São Tomé and Príncipe began reporting monetary and balance of payments statistics in the IMF's International Financial Statistics (IFS) in March 1998 and has participated in the Fund's General Data Dissemination System (GDDS) since April 20, 2004. In addition, the country has benefited since 2003 from technical assistance provided by the Statistics Department (STA) under the GDDS project for Lusophone Africa.

\section{National accounts}

23. Since the mid-1990s, some technical assistance for the compilation of the national accounts has been provided by the World Bank and the United Nations Development Program (UNDP). As a result, the national account estimates compiled for 1994-97 followed to some extent the 1993 System of National Accounts (1993 SNA) methodology, and incorporated improvements in the source data, compilation methodology, and statistical techniques. However, improvements could not be sustained and beginning in 1998, the quality of national accounts data deteriorated mainly due to a decline in the availability of source data and dwindling resources allocated to statistical work. Since 1998, only preliminary flash estimates of GDP at current and constant prices have been compiled using industry coefficients and growth rate estimates.

24. Since 2003, the Fund has also provided technical assistance in the context of the GDDS Lusophone project. With this assistance, the National Institute of Statistics (INE) has made considerable efforts, mainly to improve source data. The INE has approved classification systems for the compilation of national accounts that are broadly consistent with internationally accepted classifications, and efforts are underway to compile national account aggregates with an improved methodology. An important goal of this project is to elaborate a new GDP series in broad conformity with the 1993 SNA by mid-2006, and to assist in compiling a quarterly production index. The Lusophone Project has been extended by another year aiming at further improvements in the national accounts of the covered countries. However, the progress achieved in strengthening the INE's statistical capacity is very fragile mainly because of the lack of incentives to retain qualified staff and allocate adequate human, financial and computer resources.

25. The CPI is calculated monthly and reported to AFR with a lag of one month. Since 1997, the compilation of the CPI has been based on a household survey, financed by the 
UNDP. Price surveys only cover the capital, although the authorities intend to develop regional price series.

26. In 2003, the authorities completed the first comprehensive survey of unemployment, with assistance from the World Bank, which provides a detailed breakdown of unemployment by age, gender and geographic location.

\section{Government finance statistics}

27. A summary of fiscal data is compiled on a monthly basis, and reported to AFR with a lag of about three weeks. The main weaknesses in the data reported are found in: (i) expenditures on projects financed by donors, which are not adequately monitored by the Ministry of Finance (MOF); and (ii) the below-the-line financing items are not yet sufficiently reliable.

28. In the context of the GDDS Lusophone project, the first government finance statistics (GFS) technical assistance mission was undertaken in October 2004. Its main objective was to assist the MOF in compiling and disseminating GFS for the general government in accordance with the IMF's Government Finance Statistics Manual 2001(GFSM 2001). An immediate objective of this project is to enhance the usefulness of existing fiscal data for the formulation, implementation, and monitoring of fiscal policy in São Tomé and Príncipe.

29. The mission prepared tables bridging the national budget classifications and those recommended in the GFSM 2001. These bridge tables are to be used to compile GFS for reporting to STA and AFR, as well as for policy analysis within the MOF. Given the importance of the oil-related revenues, the mission also reviewed in detail the classification of the oil revenues regulated under the recently approved Oil Revenue Management Law, and made recommendations on the classification of these transactions. The mission's report contains an action plan for implementing its recommendations that were agreed with the MOF officials. It would be useful to follow up with the authorities on progress with the implementation of these recommendations.

30. Currently, no GFS are reported to STA for publication in the Government Finance Statistics Yearbook (GFSY) and International Financial Statistics (IFS).

\section{Monetary statistics}

31. An STA mission provided technical assistance on monetary and statistics in December 2004. Since then, the lags with which monthly monetary data are being reported by the Central Bank of São Tomé and Principe (BCSTP) to STA have been reduced to a twomonth lag.

32. The mission conducted a review of the current collection and compilation procedures 
of monetary statistics compiled by BCSTP. It developed a work program to address shortcomings, including the statistical issues recently raised by the Safeguards Assessment mission, and provided training to the CBSTP staff to facilitate the compilation of the monetary statistics.

33. The mission found that the CBSTP monthly balance sheets were adequate to compile monetary statistics in line with the Monetary and Financial Statistics Manual (MFSM). Although the accounting treatment of revaluation adjustments (holding gains) is not consistent with recommended practices, data are available for the correct reclassification of the revaluation adjustments. The plan of accounts of the other depository corporations is not fully consistent with the $M F S M$, particularly for recently created banks.

\section{External sector}

34. The authorities record the rates of the official exchange bureaus, commercial banks, and the parallel market. These rates are available on the internet with a one-day lag. The official rate is the simple average of the market rates for the previous day.

35. With respect to merchandise trade, there are significant weaknesses in the source data collection. Some transactions, including some imports related to investment, are not being fully captured in the balance of payments. Monthly data on the main exports and imports are reported to AFR regularly, but unit prices and volumes of exports are only occasionally included.

36. Balance of payments statistics are compiled by the BCSTP. Annual balance of payments data are reported to STA and published in the IFS, albeit with long lags and subject to substantial revisions.

37. In the context of the GDDS Lusophone project, STA launched a technical assistance program to improve balance of payments compilation and dissemination. The GDDS project targets a substantial improvement in the source data, including the response rate to the surveys, and in the methodology for compiling the balance of payments consistent with the Fund's Balance of Payments Manual. The first mission, in June 2003, reviewed the shortcomings of current and financial account transactions that are reported, and concluded that the weaknesses were mainly due to the under coverage of several important sectors, including foreign direct investment and commercial banks' reports on international transactions.

38. The follow-up mission undertaken in early June 2004 found that despite the effort to implement the previous mission's recommendations, several measures, in particular those related to improving source data, were only partially implemented or, in some cases, not implemented at all. As a result, inconsistencies in the classification of BOP operations still persisted and the disaggregated BOP components continued to be largely estimated because of weak source data. Resource constraints, particularly with regard to qualified staff, 
contribute to the lack of improvement in the response rate to the balance of payments surveys.

39. The mission recommended improving the coordination and data sharing among data producing agencies; intensifying contacts with survey respondents, and using alternative sources in order to substantially improve the source data to compile the balance of payments.

40. The authorities have built on previous efforts to strengthen debt data management. A new unit was created in late 2003 within the Ministry of Finance, incorporating staff from the debt department of the BCSTP. New staff, including a director, has been appointed, which should improve the monitoring of debt statistics. However, since 2003 a technical assistance mission on balance of payments statistics noted that the data available for external debt are incomplete and do not conform to international guidelines. 


\section{TAble 1. SÃo Tomé And PrínCIPE: CoMmon IndiCATORS REQUIRED FOR SURVEILLANCE \\ (As of January 12, 2006)}

\begin{tabular}{|c|c|c|c|c|c|}
\hline & $\begin{array}{l}\text { Date of latest } \\
\text { observation }\end{array}$ & $\begin{array}{l}\text { Date } \\
\text { received }\end{array}$ & $\begin{array}{c}\text { Frequency } \\
\text { of } \\
{ }_{\text {Data }}^{6}\end{array}$ & $\begin{array}{l}\text { Frequency } \\
\text { of } \\
\text { Reporting }\end{array}$ & $\begin{array}{l}\text { Frequency of } \\
\text { publication }\end{array}$ \\
\hline Exchange Rates & Dec 2005 & Jan 2006 & $\mathrm{D}$ & $\mathrm{D}$ & $\mathrm{D}$ \\
\hline $\begin{array}{l}\text { International Reserve Assets and Reserve Liabilities } \\
\text { of the Monetary Authorities } 1\end{array}$ & Nov 2005 & Dec 2005 & M & M & M \\
\hline Reserve/Base Money & Nov 2005 & Dec 2005 & M & M & M \\
\hline Broad Money & Nov 2005 & Dec 2005 & M & M & M \\
\hline Central Bank Balance Sheet & Nov 2005 & Dec 2005 & M & M & $\mathrm{M}$ \\
\hline Consolidated Balance Sheet of the Banking System & Nov 2005 & Dec 2005 & M & M & M \\
\hline Interest Rates 2 & Nov 2005 & Dec 2005 & M & M & M \\
\hline Consumer Price Index & Nov 2005 & Dec 2005 & M & M & M \\
\hline $\begin{array}{l}\text { Revenue, Expenditure, Balance and Composition of } \\
\text { Financing }{ }^{3} \text { - General Government }\end{array}$ & NA & NA & NA & NA & NA \\
\hline $\begin{array}{l}\text { Revenue, Expenditure, Balance and Composition of } \\
\text { Financing }{ }^{3} \text { - Central Government }\end{array}$ & Nov 2005 & Dec 2005 & M & M & M \\
\hline $\begin{array}{l}\text { Stocks of Central Government and Central } \\
\text { Government-Guaranteed Debt } 5\end{array}$ & Dec. 2004 & May 2005 & A & I & A \\
\hline External Current Account Balance & Dec. 2004 & May 2005 & A & I & A \\
\hline Exports and Imports of Goods and Services & Dec 2005 & Jan 2006 & A & I & A \\
\hline GDP/GNP & 2004 & May 2005 & A & I & A \\
\hline Gross External Debt & Dec. 2004 & May 2005 & Q & Q & I \\
\hline
\end{tabular}




\section{Statement by the IMF Staff Representative March 6, 2006}

The following information has become available since the issuance of the staff report for the 2005 Article IV consultation and First Review Under the Three-Year Arrangement Under the Poverty Reduction and Growth Facility. The thrust of the staff appraisal remains unchanged.

1. Recent developments under the program

- $\quad$ Based on preliminary information, all quantitative performance criteria for end-December 2005 appear to have been met. The monthly inflation rate for January 2006 (2.7 percent) continued to be relatively high reflecting seasonal factors and the pass-through of the rather large depreciation of the dobra during the last quarter of 2005. However, the exchange rate was stable in January and February 2006.

- $\quad$ On the fiscal side, revenue was slightly below the program target on account of delays in payment of fishing licenses granted to the European Union, while current domestic primary expenditure was lower than programmed resulting in a smaller domestic primary deficit than under the program. All domestic payment arrears incurred in late 2005 were eliminated in the first two weeks of 2006.

\section{Oil sector developments}

- Exploratory drillings: Chevron-Texaco, the designated operator for Block 1 in the Joint Development Zone administered with Nigeria, started exploratory drilling operations on January 14, 2006. The offshore drilling is estimated to last 40-60 days. Chevron-Texaco has indicated that, if significant amounts of crude are discovered, commercial production could begin in 2010 .

- $\quad$ Licensing round of oil Blocks 2-6: The Sãotomean authorities have informed the staff about the outcome of the Joint Ministerial Committee (JMC) meeting held in Abuja in early February 2006.

- The Sãotomean delegation raised the concerns noted in the report from the Attorney General's Office of São Tomé and Príncipe about lack of transparency and irregularities in the bidding process of Blocks 2-6, which were mentioned in paragraph 14 of EBS/06/23.

The Nigerian delegation indicated that they would reply in writing to the Attorney General's report, while noting that they disagreed with the report's conclusions. 
- The Sãotomean authorities stated that they intend to strengthen transparency of oil sector transactions at three different levels: first, the government will support the country's Attorney General's Office in conducting further investigations and needed legal actions; second, the Sãotomean delegates at the JMC will propose that the Attorney General's recommendations regarding the implementation of international standards be applied to future oil bidding rounds; and third, São Tomé and Príncipe will work towards the implementation of Extractive Industries Transparency Initiative (EITI) principles, as stated in the Abuja Declaration.

- Status of Production Sharing Agreements (PSAs): The signature of the PSAs on Blocks 3 and 4, between the Joint Development Agency and the designated oil consortiums, is expected during the coming weeks. The signature of PSAs for other blocks is expected in the next 2-3 months.

3. Poverty Reduction Strategy Paper (PRSP): The Sãotomean authorities submitted the first PRSP annual progress report to the Fund and the World Bank on February 24, 2006. The preparation of the PRSP annual progress report-which is currently being translated into English - and the related joint staff advisory note (JSAN) constitute a floating completion point trigger as specified in the HIPC Decision Point document of December 2000 (EBS/00/254). The JSAN will be circulated to the Board in conjunction with the staff report for the Second Review under the existing PRGF arrangement in June/July 2006. It is expected that the HIPC Completion Point document would also be circulated to the Board at that time, provided that all floating completion point triggers are met. The mission on the Second Review under the PRGF arrangement is scheduled for early-May, once a new government is in place, following the Parliamentary elections in late-March 2006. 


\section{INTERNATIONAL MONETARY FUND EXTERNAL RELATIONS Public Information Notice DEPARTMENT}

Public Information Notice (PIN) No. 06/36 FOR IMMEDIATE RELEASE March 29, 2006
International Monetary Fund

$70019^{\text {th }}$ Street, NW

Washington, D.C. 20431 USA

\section{IMF Executive Board Concludes 2005 Article IV Consultation with São Tomé and Príncipe}

On March 6, 2006, the Executive Board of the International Monetary Fund (IMF) concluded the Article IV consultation with São Tomé and Príncipe. ${ }^{1}$

\section{Background}

Following a decade of large macroeconomic imbalances and state intervention in the economy, the Sãotomean authorities have pursued economic reforms since 1998 that have helped increase real GDP growth, lower inflation, and push forward key structural reforms. Financial aid from international donors increased substantially in support of these reforms. At the same time, a successful implementation of a Staff Monitored Program in 1999 led to the approval of a three-year arrangement under the Poverty Reduction and Growth Facility (PRGF) in April 2000. São Tomé and Príncipe reached the Decision point under the Heavily Indebted Poor Countries (HIPC) Initiative in late December 2000.

However, fiscal performance has been uneven over the years, reflecting expenditure pressures arising from the domestic political cycle and anticipated large oil signature bonuses. In 2001, as presidential elections approached, the government failed to meet a number of fiscal and monetary targets under the Fund-supported program. Fiscal management improved in 2002, but it deteriorated again in 2003 as the government raised social spending significantly. In 2004, the fiscal position deteriorated even further as the government increased expenditures to

\footnotetext{
${ }^{1}$ Under Article IV of the IMF's Articles of Agreement, the IMF holds bilateral discussions with members, usually every year. A staff team visits the country, collects economic and financial information, and discusses with officials the country's economic developments and policies. On return to headquarters, the staff prepares a report, which forms the basis for discussion by the Executive Board. At the conclusion of the discussion, the Managing Director, as Chairman of the Board, summarizes the views of Executive Directors, and this summary is transmitted to the country's authorities.
} 
unsustainable levels in anticipation of a large oil signature bonus, which was only realized in July 2005. The fiscal gaps were financed mainly through external borrowing, central bank financing, and the accumulation of arrears. The current PRGF arrangement, approved by the Executive Board on August 1, 2005, seeks to support the government's efforts to address macroeconomic imbalances, deepen structural reforms, and set the conditions for sustained economic growth and poverty alleviation.

In 2005, economic growth remained strong, although inflation picked up against the background of a sharp increase in international oil prices that were passed through to domestic consumers. Fiscal consolidation advanced in line with the program, and the fiscal targets for end-2005 are likely to have been met. Broad money grew rapidly on account of resilient economic growth and an increase in the central bank's Net International Reserve position. The managed floating exchange rate regime has served the country well, allowing the authorities to address the volatility of external inflows and cushion exogenous shocks. The import coverage of reserves remains above 3.5 months of imports of goods and services.

Prospects for 2006 remain favorable, with an expectation of strong growth, lower inflation, and a relatively favorable external position. In the fiscal area, the 2006 program seeks to consolidate the measures launched in 2005, including efforts to address tax arrears and overhaul customs administration procedures. A better control of the growth of current expenditure will help to change the composition of government spending in favor of pro-poor expenditure. Monetary policy will be consistent with a deceleration of inflation. To foster private sector growth in the non-oil economy, structural reform efforts will continue, including actions to address the financial position of the water and electricity company (EMAE).

\section{Executive Board Assessment}

Executive Directors welcomed the commitment of the Sãotomean authorities to continue addressing macroeconomic imbalances, while strengthening the conditions for sustained economic growth and poverty reduction. Over the medium term, a main challenge will be to develop strong institutions to secure a transparent management of oil revenue and to secure a proper execution of the government's Poverty Reduction Strategy Paper (PRSP) that would strengthen growth in the non-oil economy and support the attainment of the Millennium Development Goals (MDGs). Lasting and enhanced donor support to finance the PRSP will be vital in this regard.

Directors commended the satisfactory performance under the PRGF-supported program. In particular, they welcomed the authorities' decision to sustain the process of fiscal consolidation. They commended the government's efforts to increase tax revenue and reduce wasteful expenditure, while increasing the share of pro-poor expenditure in line with the PRSP. Fiscal consolidation will also be supported by a modernized budget 
and public expenditure management system (SIGFE), and the reform of the auditing practices of the general government and public enterprise financial operations.

Directors observed that the monetary policy stance remained broadly adequate, supporting the achievement of the authorities' inflation target and safeguarding the central bank's international reserve position. Directors encouraged the authorities to continue the judicious mix between foreign exchange sales and the issuance of liquidityabsorbing instruments. They welcomed the authorities' commitment to keep the central bank reference rate above the inflation rate and to improve the coordination of fiscal and monetary policies to better forecast liquidity. Directors supported continued technical assistance by the Fund in the area of indirect monetary policy management.

Directors considered that São Tomé and Príncipe's managed floating exchange rate system remains appropriate, allowing the authorities to address the volatility of external inflows and cushion exogenous shocks. In this context, Directors commended the authorities for improving the foreign exchange auction system to strengthen the price setting mechanism.

Directors welcomed the improvements in banking sector supervision and underscored the need for effective implementation of the measures regarding the licensing of new banks and the development of on-site and off-site prudential indicators. Directors underscored the importance of decisive action with respect to weak banks, including possible recapitalization or closure, which would play a critical role in developing a sound domestic banking system.

Directors noted that São Tomé and Príncipe has a relatively open trade regime and welcomed the termination in 2005 of the only remaining nontariff barrier protecting the domestic telecommunications company from competition.

Directors urged the authorities to accelerate structural reforms and achieve full transparency in oil revenue management. In this respect, they emphasized the need to implement during 2006 the action plan to improve the finances of the water and electricity company and complete the feasibility studies of the airport and seaport authorities. To improve the business climate, Directors recommended to eliminate administrative barriers for start-up businesses, establish tribunals of arbitration, and approve new codes for personal and corporate income taxation. They stressed that the publication of the Production Sharing Agreement signed between the Joint Development Agency and the selected oil operators and the participation in the Extractive Industries Transparency Initiative would be key elements toward securing accountability and good governance in the nascent oil sector. 
Directors encouraged the authorities to improve the coverage, timeliness, and periodicity of basic economic statistics.

Directors welcomed the authorities' stated intention to accept the obligations of Article VIII, Sections 2 (a), 3 and 4 in the near future and looked forward to the staff's findings in the ongoing review of the exchange system, expected for the next review under the PRGF arrangement.

Public Information Notices (PINs) form part of the IMF's efforts to promote transparency of the IMF's views and analysis of economic developments and policies. With the consent of the country (or countries) concerned, PINs are issued after Executive Board discussions of Article IV consultations with member countries, of its surveillance of developments at the regional level, of post-program monitoring, and of ex post assessments of member countries with longer-term program engagements. PINs are also issued after Executive Board discussions of general policy matters, unless otherwise decided by the Executive Board in a particular case. 
São Tome and Principe: Selected Economic Indicators

\begin{tabular}{|c|c|c|c|c|}
\hline & 2003 & 2004 & $\begin{array}{r}2005 \\
\text { Est. }\end{array}$ & $\begin{array}{l}2006 \\
\text { Proj. }\end{array}$ \\
\hline & \multicolumn{3}{|c|}{ (Annual percentage changes) } & \\
\hline Real GDP & 4.0 & 3.8 & 3.8 & 4.5 \\
\hline Consumer Prices (end of period) & 10.2 & 15.2 & 17.0 & 13.0 \\
\hline Consumer Prices (annual average) & 9.6 & 12.8 & 16.2 & 14.8 \\
\hline Real effective exchange rate $1 /$ & -10.0 & -1.9 & 4.4 & $\ldots$ \\
\hline \multirow[t]{2}{*}{ Terms of trade } & -10.1 & -18.8 & -5.2 & -4.0 \\
\hline & \multicolumn{3}{|c|}{ (In percent of GDP) } & \\
\hline Gross domestic investment & 36.1 & 35.2 & 36.0 & 42.2 \\
\hline Gross domestic savings & 15.4 & 16.7 & 4.3 & 14.2 \\
\hline \multirow[t]{2}{*}{ Public savings } & 13.4 & 6.8 & 11.6 & 15.5 \\
\hline & \multicolumn{4}{|c|}{ (In millions of US\$, unless otherwise specified) } \\
\hline Exports (f.o.b.) & 6.6 & 3.5 & 3.8 & 4.0 \\
\hline Imports (f.o.b.) & 33.6 & 36.0 & 42.1 & 44.0 \\
\hline Current account balance $2 /$ & -33.5 & -37.9 & -41.6 & -42.5 \\
\hline $\begin{array}{l}\text { Current account balance (in percent } \\
\text { of GDP) } 2 /\end{array}$ & -56.7 & -58.9 & -59.2 & -59.8 \\
\hline Gross official reserves $3 /$ & 4.8 & 3.5 & 3.7 & 3.5 \\
\hline Overall balance 4/ & 1.9 & -5.0 & 26.6 & 21.1 \\
\hline \multirow[t]{2}{*}{ Net present value of total debt $5 /$} & 463.5 & 500.7 & 499.5 & 315.5 \\
\hline & \multicolumn{4}{|c|}{ (In percent of GDP, unless otherwise specified) } \\
\hline Total revenue and grants $4 /$ & 58.1 & 60.6 & 129.7 & 139.4 \\
\hline Total expenditure & 75.1 & 87.2 & 72.8 & 79.7 \\
\hline Non-interest current expenditure & 28.4 & 41.7 & 38.0 & 38.3 \\
\hline $\begin{array}{l}\text { Overall fiscal balance, } \\
\text { excluding grants } 4 /\end{array}$ & -49.5 & -58.8 & 32.3 & 30.6 \\
\hline $\begin{array}{l}\text { Overall fiscal balance, } \\
\text { including grants } 4 /\end{array}$ & -17.0 & -26.6 & 56.9 & 59.6 \\
\hline Budget grants & 32.5 & 32.2 & 24.6 & 29.0 \\
\hline Change in broad money (in percent) & 41.8 & 7.4 & 26.1 & 16.1 \\
\hline Interest rate (in percent) 6/ & 10.6 & 10.6 & 12.5 & $\ldots$ \\
\hline
\end{tabular}

Sources: Sãotomean authorities; and IMF staff estimates and projections.

$1 /(+)=$ appreciation. For 2003, data are through October.

2/ Excluding official transfers.

$3 /$ In months of following year's non-oil imports of goods and nonfactor services.

4/ Includes oil signature bonuses in 2005 and 2006.

$5 /$ In percent of exports of goods and services, calculated as a three-year backward-looking average (e.g., average over 2001-2003 for exports in 2003).

6/ Commercial bank deposit rate (90-180 days), end of period. 
Press Release No. 06/44

FOR IMMEDIATE RELEASE

March 7, 2006
International Monetary Fund

Washington, D.C. 20431 USA

\section{IMF Executive Board Completes First Review of São Tomé and Príncipe's Three-Year PRGF Arrangement and Approves US\$600,000 Disbursement}

The Executive Board of the International Monetary Fund (IMF) has completed the first review of São Tomé and Príncipe's economic performance under a three-year Poverty Reduction and Growth Facility (PRGF) arrangement. The completion of the review enables the release of an amount equivalent to SDR 0.4 million (about US\$600,000), which would bring total disbursements under the arrangement to SDR 0.8 million (about US\$1.2 million).

The Executive Board approved the three-year arrangement on August 1, 2005 (see Press Release No. 05/187), for a total amount of SDR 2.96 million (about US $\$ 4.26$ million) to support the government's economic program for 2005-2007.

In commenting on the Executive Board's discussion on São Tomé and Príncipe, on March 6, 2006 Mr. Agustín Carstens, Deputy Managing Director and Acting Chair, stated:

"The authorities of São Tomé and Príncipe are committed to continue to address macroeconomic imbalances, while strengthening the conditions for sustained economic growth and poverty alleviation. Over the medium term, a main challenge will be to develop strong institutions to secure a transparent management of oil revenue, while implementing the government's Poverty Reduction Strategy Paper (PRSP) to strengthen growth in the non-oil economy and advance towards attaining the Millennium Development Goals. Donor support also has an important role to play in this process.

"Performance under their PRGF-supported program has been satisfactory. The authorities are to be commended for their intention to sustain the process of fiscal consolidation that started in 2005. Within this strategy, the authorities intend to increase tax revenue, reduce wasteful expenditure, and increase the share of pro-poor expenditure in line with the PRSP. Fiscal consolidation will also be enhanced by the implementation of a fully integrated, budget and public expenditure management system, and the reform of the auditing practices of the general government and public enterprise financial operations.

"The overall stance of monetary policy remains broadly adequate, with the objective to achieve the authorities' inflation target and safeguarding the central bank's international reserve position. To control liquidity, the authorities will continue their judicious mix between foreign exchange sales and the issuance of liquidity absorbing instruments. The authorities are committed to

Washington, D.C. 20431 • Telephone 202-623-7100 • Fax 202-623-6772 • www.imf.org 
keeping the central bank reference rate above the inflation rate and improving the coordination of fiscal and monetary policies.

"São Tomé and Príncipe's managed floating exchange rate system remains appropriate, as it allows the monetary authorities to address the volatility of external inflows and cushion exogenous shocks. The improvement of the foreign exchange auction system is commendable as it strengthens the price mechanism of the managed floating exchange rate system.

"The efforts to enhance the banking sector supervisory capacity are welcome. Critical in this regard will be effective implementation of the procedures regarding licensing of new banks and the development of on-site and off-site prudential regulation indicators. Decisive action regarding weak banks will be also important to foster the development of an efficient and sound domestic banking system.

"Key challenges for the future are to accelerate structural reforms and achieve full transparency in oil revenue management. Publication of all critical information regarding the management of oil revenue will be important in securing accountability and good governance in the sector. In addition, there is a need for steadfast implementation of the action plan to improve the finances of the water and electricity company and the completion of the feasibility studies of the airport and seaport authorities. Necessary measures to improve the business climate will include the elimination of administrative barriers to start-up businesses, the establishing of tribunals of arbitration, and the approval of new codes for personal and corporate income taxation.

"The authorities' commitment to improve the coverage, timeliness, and periodicity of basic macroeconomic statistics is most welcome," Mr. Carstens said.

The PRGF is the IMF's concessional facility for low-income countries. PRGF-supported programs are based on country-owned poverty reduction strategies adopted in a participatory process involving civil society and development partners and articulated in the Poverty Reduction Strategy Paper (PRSP). This is intended to ensure that PRGF-supported programs are consistent with a comprehensive framework for macroeconomic, structural, and social policies to foster growth and reduce poverty. PRGF loans carry an annual interest rate of 0.5 percent and are repayable over 10 years with a $5^{1 / 2}$-year grace period on principal payments. 


\section{Statement by Damian Ondo Mañe, Executive Director for the Democratic Republic of São Tomé and Príncipe March 6, 2006}

On behalf of my Saotomean authorities, I would like to express my appreciation to Management and the staff for their continued assistance and advice to São Tomé and Príncipe. My authorities are of the view that the staff report describes well the economic developments in São Tomé and Príncipe as well as the challenges facing the country. In addition they think that the Selected Issues paper highlights adequately relevant topical oilrelated issues.

\section{I - Recent Economic Developments}

Macroeconomic performance has been satisfactory in 2005. Output is estimated to have grown by 3.8 percent last year, mostly driven by strong demand, despite an adverse external environment. Inflation, however, rose on account of higher international oil prices and the depreciation of the dobra. Indeed, it should be noted that over the last year, my authorities adjusted local petroleum prices to reflect changes in international oil prices. Consequently, the electrical company EMAE, which petroleum product is an intermediate good for the production of electricity, also raised its tariffs to cover the additional costs. As a result, inflation was above the program target by 2 percentage points, at 17 percent in December 2005.

Fiscal policy implementation has also improved, as the authorities pursued their fiscal consolidation policy over the course of 2005, despite strong social demands, and they managed to achieve the fiscal objectives set for 2005. In particular, large cuts were made in domestic primary spending to cope with the shortfalls in tax revenues and the increase in the minimum monthly salary in the public sector from US\$ 31 to US\$ 40. As a result of spending restraint, the fiscal deficit was slightly lower-than-programmed. Fiscal reforms progressed well as all draft new codes on personal income, corporate taxation and tax tribunals (structural performance criteria) as well as the new guidelines on urban property taxation and the new legislation on inheritance taxes (benchmark criteria) have been submitted to the National Assembly for approval. Public expenditure and external debt management are being improved with the assistance of the World Bank.

Monetary policy aimed at controlling the inflation surge, with notably increases in the bank reference rate and banks' reserve requirements. Preliminary information indicates that this move has been successful in lowering bank credit to the economy. The dobra depreciation occurred following the technical changes to the central bank foreign exchange auction in the last quarter of 2005. Reforms to improve the operations of the central bank and the conduct of monetary policy advanced well, with the assistance of the Fund. The BCSTP has posted on its internet website the external audit of its 2003 and 2004 financial statements, as agreed 
in the context of the safeguards assessment of the BCSTP conducted by the Fund's Finance Department in early 2004. Publication of the audited financial statements will become the norm for the central bank from now on.

The resumption of the tourism activity, after a period of sluggishness in the aftermath of the Coup in 2003, as well as lower payments for technical assistance contributed to offset the deterioration of the trade balance, thus keeping the current account balance (excluding official transfers) in 2005 at the same level as that of 2004, at around 59 percent of GDP. The overall external balance, however, is estimated to improve due mainly to the receipt of oil signature bonuses, which are held in an account, the National Oil Account (NOA), at the central bank. The latter's gross international reserves, excluding the above-mentioned account, remained at around 3.5 months of imports by end-2005.

For years now, São Tomé and Príncipe has embarked in a wide range of structural reforms aiming at promoting private sector and strengthening the business environment. A new investment code that provides for equal treatment of domestic and foreign investors, which is consistent with the new code on corporate taxation is before the National Assembly for examination. There has been substantial progress in the public enterprise and judicial sectors reform. A comprehensive strategy has been launched to fundamentally improve the financial position of the water and electricity company (EMAE). To this end, in addition to the tariffs above-mentioned, a timetable for the reduction of outstanding payments arrears to EMAE has been established. In consultation with the World Bank, work is progressing regarding the preparation of terms of reference for the conduct of feasibility studies on the public enterprises that administer the airports (ENASA) and seaports (ENAPORT). The longlasting non-tariff barrier related to the privately-managed telecommunications company (CST) has been eliminated in December 2005, thus opening the sector to new operators. Regulations for an effective functioning of the telecom sector are being finalized, which will allow for the launching of bidding rounds for different telecommunications services. The submission to the National Assembly of a legislation criminalizing money laundering and the financing of terrorism has been delayed, due to the need to update related pieces of legislation. With the assistance of Fund's LEG and MFD, the government intends to continue a review of these pieces of legislation and will submit this legislation in the course of the year.

Turning to the debt situation, São Tomé and Príncipe is current with its obligations vis-à-vis the multilateral creditors and has cleared its arrears with Paris Club creditors as part of the Paris Club debt rescheduling agreement reached in September 2005. The authorities are currently in the process of reconciling debt records with Paris Club creditors, with the expectation of signing bilateral agreements by April 1, 2006.

Concerning the oil sector, as I stated in my Buff statement in August 2005, the institutional framework for developing the national petroleum strategy and for the supervision and regulation of the sector became operational in the first half of 2004, with the settings of 
National Petroleum Council (NPC) and the National Petroleum Agency (NPA) as well as the adoption of an Oil Revenue Management Law (ORML). The NPA is receiving technical assistance to develop advisory capacity and has launched its internet website in December 2005. Public information campaigns on oil-related issues took place through national forums with the civil society in 2005. An overall strategy to develop the oil sector has been completed in mid-2005 and is to be submitted to the National Assembly.

My authorities remain committed to ensuring transparency and good governance in the management of the oil sector. Following the recent disclosure by the Attorney General's Office of São Tomé and Príncipe of irregularities in the awarding of oil Blocks 2-6, my authorities have stated that they will support the Attorney General's Office in conducting further investigations and needed legal actions on this issue. In addition, they will propose that the Attorney General's recommendations regarding the implementation of international standards be applied to future oil bidding rounds while working towards the implementation of Extractive Industries Transparency Initiative (EITI) principles.

Performance under the PRGF has been satisfactory, as all eight quantitative performance criteria set for end-September 2005 and all structural performance criteria set for end-July 2005 were met. In addition, all structural benchmarks for end-September 2005 have been met, with the exception of the one on AML/CFT. In light of this satisfactory performance, my authorities request the completion of the first review under the PRGF.

\section{II - Program for the Medium-Term and Policies for 2006}

The medium-term macroeconomic outlook for São Tomé and Príncipe is favorable, following particularly the recent developments in the oil sector. Real GDP is expected to increase by at least 5 percent on average over the next three years, on account of higher investment in several sectors in anticipation of the oil era. However, as I stated in previous meetings, São Tomé and Príncipe is not an oil-producing country yet and, therefore, it will very likely remain a low-income country at least until oil production starts, which is expected in 2012. Thus, São Tomé and Príncipe, as any other low-income country, is still facing - and will continue to face in the near to medium term - daunting challenges. These include among others, consolidating macroeconomic stability, while addressing pressing social needs, as highlighted by the recent cholera outbreak in the archipelago. My authorities are of the view that part of oil bonuses from the awarding of oil blocks could be used to complement foreign assistance in satisfying these needs, consistent with the PRSP. They do not lose sight of the need to avoid Dutch disease and, accordingly, they remain committed to a proper and transparent use of these resources.

In 2006, the economic activity is expected to grow by 4.5 percent and inflation to decline to 13 percent from 17 percent in 2005, owing to a prudent monetary policy. Overall, my authorities intend to pursue their prudent macroeconomic and structural policies. 
Fiscal policy takes into account recent developments in the local oil sector and in the tax reform, and gives more weight to pro-poor spending. Specifically, revenues (excluding oil bonuses) are expected to increase by 2 percentage points of GDP with the coming into effect of several revenue-enhancing measures adopted last year. Expenditures will increase by 5 percentage points, reflecting mainly higher investment spending in agriculture and fisheries, and greater outlays in social sectors on one hand, and continued control of the wage bill on the other hand. On the wage bill, my authorities will enforce a strict management of the payroll through the activation of the civil servants' register that has been recently developed with the UNDP. Further increase in the wage bill will be implemented once a new government is appointed following the holding of parliamentary, regional and presidential elections scheduled this year. As noted above, the larger fiscal deficit expected in 2006 will be financed by part of the oil bonuses and foreign assistance.

In order to improve public finance management, a fully integrated, computerized budget and public expenditure management system (SIGFE), developed with assistance of the World Bank is slated to replace the existing system by end-December 2006 (performance criterion). My authorities also intend to launch a thorough reform of the Inspectorate-General of Finance. They will amend internal control laws and administrative regulations governing the IGF's actions by end-March 2006 (structural benchmark), with the aim of increasing the IGF's powers regarding the oversight and auditing of the financial operations of the central government, public enterprises, and local governments. By end-June 2006, the government will establish a database to record and monitor follow-up actions addressing problems identified by the IGF in each inspected sector, including the applicable sanctions and penalties (structural benchmark).

Monetary policy will aim at bringing inflation to a lower level by end-2006, through notably ceilings on the expansion of the central bank's domestic assets and banking sector credit to the government. Nevertheless, the central bank (BCSTP) stands ready to reassess its monetary policy stance whenever it deems necessary. In particular, the central bank will keep its reference interest rate above the inflation rate and will use its available instruments to address liquidity concerns. The BCSTP will pursue the strengthening of its operational capacities, with technical assistance from Fund's MFD on the development of additional instruments, in the areas of banking supervision, development of interbank money markets, and foreign exchange auctions. The BCSTP remains committed to the continuation of the existing managed float exchange rate arrangement, which has allowed the monetary authority to appropriately address the volatility of external inflows and outflows.

My authorities intend to pursue their structural reforms aiming at improving the business climate and supporting private sector entrepreneurship. In particular, in order to alleviate administrative burdens on start-ups, my Saotomean authorities, along with the IFC advice and assistance, plan to address ways to modernize the business registration process, through notably the establishment of a one-stop shop. A deadline for the completion of the feasibility 
studies of ENASA and ENAPORT will be set this year in consultation with the Fund and World Bank staffs.

My authorities also intend to pursue efforts to increase transparency. In the oil sector, they will ensure a regular updating of the newly-established internet website of the NPA. In addition, in cooperation with the World Bank, they are developing an implementation manual/handbook of the oil revenue management law that will be posted on the websites of the National Assembly and the NPA by the end of the first quarter of 2006.

Regarding poverty reduction, my authorities have recently submitted the first PRSP annual progress report to the Fund and the World Bank, which has been prepared in a participatory process, in collaboration with the country's main donors and civil society. The preparation of the PRSP annual report constitutes one of the floating completion point triggers specified under in the HIPC Decision Point Document of December 2000. The completion point is expected to be reached in mid-2006.

\section{III - Conclusion}

My Saotomean authorities have demonstrated great degree of ownership of their program, and they are resolved to pursue prudent macroeconomic policies and sound structural reforms over the medium-term. In particular, with the advent of the oil era, work is being done to ensure that the regulatory framework for a proper development of the oil sector and an efficient management of oil resources is in place. Nevertheless, São Tomé and Príncipe remains a low-income country, at least until oil revenue flow into its economy, and it is still facing challenges inherent to this group of countries. Addressing these obstacles during this transition phase toward the oil era will require continuous support from the international community. My authorities are particularly grateful to the latter for its support so far through notably the debt rescheduling agreement with the Paris Club in September 2005 and the pledges for the Priority Actions Program at the recent roundtable meeting with donors that took place in Brussels last December. They look forward to the completion point under the HIPC Initiative and, subsequently, to immediate debt reduction under the MDRI this year. My authorities are of the view that all these actions will be critical to a successful implementation of their growth-enhancing and poverty reduction strategy. 